\title{
Aliens on the Svalbard beach
}

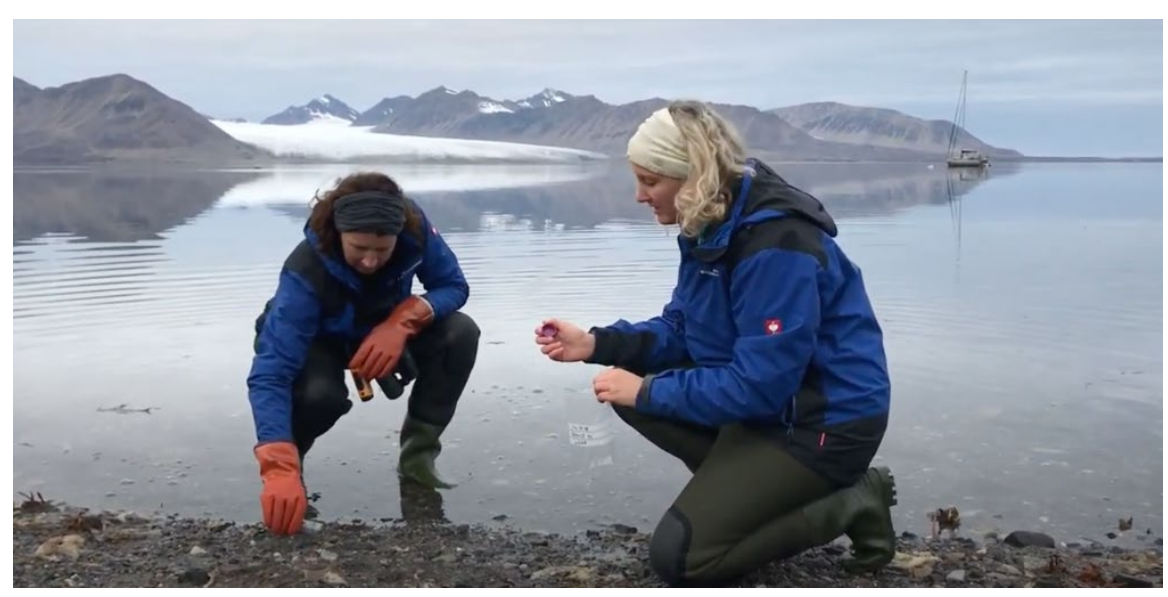

Author(s): Anneke van den Brink, Paul Renaud, Piotr Kuklinski, Sander Glorius, Arjen de Groot, Ingeborg Mulder, Lech Kotwicki, Jan Marcin Węsławski, Martine van den Heuvel-Greve

Wageningen Marine Research, University Centre in Svalbard, Akvaplan-niva, Institute of Oceanology of the Polish Academy of Sciences 
Keywords: monitoring, beach, Svalbard, non-indigenous species, marine

This project was funded by the Svalbard Environmental Protection Fund (18/35 - Aliens on the beach); the Knowledge Base Programme of Wageningen University and Research (431.830.0049); the Norwegian Environmental Department, University Centre in Svalbard, Akvaplan-niva; ADAMANT project - DAINA - Polish-Lithuanian Funding Initiative, NCN 2017/27/L/NZ8/03331.

This report can be downloaded for free from https://doi.org/10.18174/544129 Wageningen Marine Research provides no printed copies of reports

Wageningen Marine Research is ISO 9001:2015 certified.

Photo cover: Wageningen Marine Research

\section{(C) Wageningen Marine Research}

Wageningen Marine Research, an institute within the legal entity Stichting Wageningen Research (a foundation under Dutch private law) represented by Dr.ir. J.T. Dijkman, Managing director

KvK nr. 09098104, WMR BTW nr. NL 8113.83.696.B16. Code BIC/SWIFT address: RABONL2U IBAN code: NL 73 RABO 0373599285
Wageningen Marine Research accepts no liability for consequential damage, nor for damage resulting from applications of the results of work or other data obtained from Wageningen Marine Research. Client indemnifies Wageningen Marine Research from claims of third parties in connection with this application.

All rights reserved. No part of this publication may be reproduced and / or published, photocopied or used in any other way without the written permission of the publisher or author. 


\section{Contents}

Aliens on the Svalbard beach

Summary

1 Introduction $\quad 6$

$\begin{array}{llr}1.1 & \text { Objectives } & 7\end{array}$

2 Methods $\quad 8$

2.1 Beaches 28

2.2 Beach habitat characteristics $\quad 10$

2.3 Sample collection $\quad 11$

2.4 Soft substrate (sediment) samples $\quad 12$

2.5 Hard substrate samples $\quad 12$

2.6 Debris samples $\quad 13$

$\begin{array}{lll}2.7 & \text { Sample analysis and data processing } & 13\end{array}$

2.7.1 Grain size analysis 13

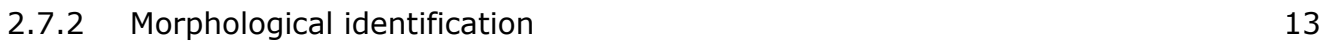

$\begin{array}{ll}\text { 2.7.3 DNA metabarcoding } & 14\end{array}$

$\begin{array}{ll}2.7 .4 & \text { Data processing }\end{array}$

2.7.1 Assigning risk levels to beaches 14

$3 \quad$ Results and discussion $\quad 15$

3.1 Beach habitat characteristics $\quad 15$

3.1.1 Beach descriptions 15

$\begin{array}{lll}3.1 .2 & \text { Grain size analysis } & 23\end{array}$

3.2 Soft substrate (sediment) 24

3.2.1 Morphological identification $\quad 24$

3.2.1 Metabarcoding $\quad 25$

3.2.1 Comparison of morphological techniques for soft substrate 26

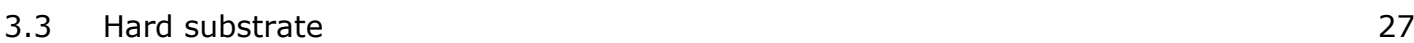

3.3.1 Morphological identification $\quad 27$

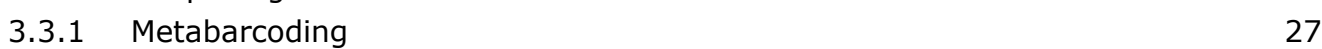

3.3.2 Comparison of identification techniques for hard substrate 29

3.4 Debris 29

3.4.1 Collected debris $\quad 29$

3.4.2 Metabarcoding 31

3.5 Metabarcoding data combined $\quad 33$

$\begin{array}{lll}3.5 .1 & \text { Species composition } & 34\end{array}$

$\begin{array}{lll}3.6 & \text { Non-indigenous species } & 36\end{array}$

$\begin{array}{lll}3.7 & \text { General discussion } & 37\end{array}$

3.7.1 Habitat classification of Svalbard beaches $\quad 37$

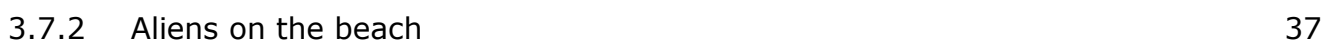

3.7.3 Metabarcoding as monitoring tool 38

$\begin{array}{lll}3.7 .4 & \text { Tourism and NIS } & 38\end{array}$

$4 \quad$ Conclusion and recommendation $\quad 40$

$\begin{array}{llr}5 & \text { Acknowledgements } & 41\end{array}$

$6 \quad$ Quality Assurance $\quad 42$ 
Justification

Annex 1. Detailed maps of sampling locations

46

Annex 2. Sampling Protocol

50 


\section{Summary}

The greater human presence in the archipelago of Svalbard through ecotourism, scientific expeditions and shipping increase the potential for the introduction of alien or non-indigenous (non-native) species (NIS). Beaches provide easy access to land-based activities for tourists and scientists, and may therefore become a port of entry for alien species. This study provides a physical and biological description of beach habitats around Svalbard and the data generated can be used as baseline information to assess changes in this remote region.

The abiotic characteristics of 16 beaches around Svalbard were described. Sediment samples of the soft substrate, hard substrate and washed-up debris were collected and analysed to determine dominant species and identify any non-indigenous species present in the area. Identification was based on visual morphological inspection and through DNA metabarcoding techniques. The beaches sampled included both those frequented by tourists and those rarely visited by humans.

The sampled beaches showed a wide range of beach types (sediment grain size, orientation, slope and exposure). Therefore, information on additional beaches around Svalbard is needed to produce a more holistic, standardised classification. Potential 'aliens' on the beach were Euterpina acutifrons (on hard substrate), Mytilus sp. (on two debris items) as well as possibly a few other species that were identified to a genus level.

Environmental DNA metabarcoding is a suitable tool for monitoring the presence of NIS in the Arctic. The use of eDNA metabarcoding provides an opportunity to sample many locations and produce a large dataset of organism identification en masse without the labour and expertise necessary for morphological identification. The use of both morphological identification and DNA metabarcoding together is recommended to produce complimentary data and a more thorough insight into the biodiversity of the beach than using one method exclusively.

Given the nature of the tourist industry on Svalbard, tourists are likely to land at beaches around most of Svalbard. The only area sampled during this study where tourists were unlikely to land was Hamberg Bukta. This ubiquity of destinations around Svalbard suggests that an introduction of NIS is likely to be spread around the archipelago through boating activities, unless quickly mitigated. Improving awareness of the vectors, risks and potential impacts of NIS in the tourism, but also fishery, shipping and research industries, is a necessary first step in implementing mitigation measures.

Species were identified on various types of debris via metabarcoding techniques suggesting that floating debris can play a role as a vector of dispersal and introduction of NIS and repatriates. It is therefore also recommended to monitor beaches where marine litter is washed upon the shore in high numbers, such as Prins Karlforland.

Regular and thorough monitoring is needed to be able to mitigate the introduction and spread of NIS. Monitoring should focus on:

- Subtidal sampling: beach samples showed a lower number of species than subtidal samples in earlier studies. It is therefore recommended to continue monitoring in subtidal compartments.

- Hotspots and points of entry: harbours with high boating activities form a likely point of entry for NIS. The same accounts for floating debris, that can play a role as a vector of both the introduction and dispersal of NIS on Svalbard.

- Complementary tools: metabarcoding providing an alternative perspective in monitoring to morphological identification. Using both tools together produces more thorough information than using either tool exclusively.

Such an approach will reduce the risk of missing an introduction and an NIS becoming established before mitigation efforts become redundant. 


\section{Introduction}

Polar beaches are considered to be among the most pristine in the world. They are, however, no longer as isolated as they once were. Climate change enables increased human activities in the Arctic, and the changing conditions allow species to survive where they previously could not. These nonindigenous species are alien to the local environment, and have the potential to become invasive, impact the local environment and irreversibly change the ecosystem. Left unchecked by natural predators, invasive species can overwhelm and dominate the local biota. Invasive species can also have economic and social impacts in the invaded area such as negative impacts on human health and decreased efficiency, and therefore profitability of marine based activities like fisheries, tourism and marine infrastructure (Molnar et al., 2008; Blackburn et al., 2014).

The likelihood of an alien species establishing in a new environment depends primarily on two factors: the ability to enter the new habitat in the first place (vector of introduction), and the ability of the organism to establish a population in the new habitat. Svalbard is an Arctic archipelago where boats are the main mode of transportation. The melting Arctic ice, as a result of climate change has opened up shipping routes that were previously inaccessible, resulting in more human traffic in the area.

Increased visitation by the public through ecotourism, scientific expeditions, the mining industry and shipping traffic puts the area at risk of both direct and indirect human impacts (e.g. Van den HeuvelGreve et al., 2016; Wiencke \& Hop, 2016; Renaud et al., 2015). In Svalbard, alien species are most likely to be introduced via biofouling of ship hulls (e.g. tourist and recreational vessels), ballast water discharges (of coal bulk carriers) (Ware et al., 2014) and floating marine debris (Węsławski \& Kotwicki, 2018).

Whether an alien species can establish in a new environment also depends on local scale interactions between physical (salinity, depth range, and the availability of a hard substrate) and biotic factors (native species richness or diversity, vacant niches, absence of enemies, and competitive abilities of resident species) (Zaiko et al., 2007). Local biodiversity is an important predictor of habitat invasibility, as a positive correlation between alien species richness and native species richness has been found in earlier studies (Zaiko et al., 2007; Van den Brink et al., in prep.).

The vulnerability of a given beach to an alien species becoming established depends partly on the habitat characteristics of the beach. Beaches provide areas where humans have easy access to land for various land-based activities, as well as areas on which debris may eventually wash up, and are therefore likely ports of entry and/or hotspots for alien species. Beaches vary greatly in habitat characteristics including substrate type, energy level, sediment chemistry, and heterogeneity. They are, therefore, differently suitable for species to colonize and survive. While an extensive study of beaches in the southern part of Svalbard was conducted in the '80s and '90s (Weslawski et al., 1993, Weslawski et al 1997, Weslawski et al 2010), beach characteristics around the rest of the island of Svalbard remain largely undocumented. Understanding the interactions between beach characteristics and species communities is important for both environmental monitoring and the management of human activities.

Observations of marine alien species in the Arctic (Molnar et al., 2008), and around Svalbard (Hopkins, 2001; Ware et al., 2016) are sparse. The presence of the snow crab, Chionoecetes opilio, was recently described in Raudfjorden (https://www.unis.no/snow-crab-arrived/), and initial results of a new study identified the presence of two non-native tunicates, Botrylloides violaceus and Molgula manhattensis, in Kongsfjorden harbour sediment based on innovative DNA metabarcoding techniques (Van den Heuvel-Greve et al., 2021). Additionally, Atlantic (southern) marine species such as mackerel and helmet jellyfish are moving northwards and are found settling in and around Svalbard (https://www.unis.no/svalbard-blue-mussels-on-the-menu/). Some species that used to be endemic to the Arctic in ancient times are returning, such as the blue mussel, Mytilus sp. (Berge et al., 2005). 
Without a monitoring programme, changes in species composition cannot be followed and the spread of more problematic alien species could go unnoticed until mitigation efforts become futile.

This is the first study that focussed on documenting the physical characteristics of beaches around the Svalbard archipelago, coupled with morphological identification and DNA metabarcoding analyses to establish a baseline of biodiversity on the different beaches. The data provided in the results can be used as a baseline for future monitoring programs, studies assessing changes in the region. The results can also be used to further promote sustainable tourism and recreation by making managers and the general public aware of the problem of alien species and show them how they can help to prevent the spreading and settlement of alien species.

\subsection{Objectives}

This project included the following objectives:

1. A habitat classification of several beaches around Svalbard according to a standardised methodology.

2. A first assessment of the presence of alien species on several beaches around Svalbard using both traditional morphological techniques and innovative DNA metabarcoding techniques.

3. A further validation of the suitability of DNA metabarcoding techniques as tool for monitoring the presence of alien species in Arctic marine environments.

4. Insight into the role of the tourist industry as potential route for the introduction of alien species on beaches around Svalbard.

5. Insight into dispersion of alien species and repatriates (such as blue mussels) via floating debris as potential route for the introduction of alien species on beaches around Svalbard.

6. Development of simple field assessment methods for future monitoring of beach habitats and alien species around Svalbard. 


\section{Methods}

\subsection{Beaches}

A total of 16 beach habitats around Svalbard were characterised, surveyed and sampled during an expedition in August-September 2018. The stretch of beach surveyed was selected based on their accessibility at the time, and in an effort to maintain a range of frequently and rarely visited areas (Table 1, Figure 1).

The beach habitat survey and sample collection took place at Svalbard in August 2018 (Figure 1). The survey was divided into two: 1) tourist beaches with more frequent visits of tourist groups, 2) beaches with none or less frequent visits by tourists or scientists.

Beaches with more frequent tourist visits on the north coast were surveyed by a team of four scientists travelling with a tourist cruise to the northern coasts of Svalbard. At each landing site for tourists during the cruise, a beach survey was conducted. During cruise time, tourists were informed and educated about settlement and impacts of marine alien species through lectures. Tourists were able to participate in the beach surveys after strict instructions and approval of the cruise staff. Beaches with a lower frequency of tourist visits on the southern coasts of Svalbard were surveyed at Storfjorden, Hornsund and Bellsund during a separate research expedition using a small sailing vessel. Additional non-tourist beaches were surveyed on Prins Karlforland and Rijpfjorden during already planned expeditions by IOPAN and UNIS respectively. Data for Prins Karlforland can also be found at the IOPAN coastal data base: https://adamant.iopan.pl/adamant/taxa_observations/ (open data).

\section{Table 1}

Beaches surveyed by the different partners during the expedition in 2018, and their type of human visitation

\begin{tabular}{|c|c|c|c|c|c|c|}
\hline Partner & $\begin{array}{l}\text { Regio } \\
\text { n }\end{array}$ & $\begin{array}{l}\text { Date } \\
2018\end{array}$ & Beach & $\mathbf{O}_{\mathbf{N}}$ & ${ }^{\circ} \mathbf{E}$ & $\begin{array}{l}\text { Human } \\
\text { Visitation }\end{array}$ \\
\hline \multirow{8}{*}{ WMR } & $\begin{array}{l}\text { Storfjord } \\
\text { en }\end{array}$ & $20-08$ & Kapp Lee-Edgeøуa & 78.5 .022 & 20.49.119 & $\begin{array}{l}\text { Tourist (av: 428, } \\
\text { max: 2008) }\end{array}$ \\
\hline & & $21-08$ & Hamberg Bukta & 77.3 .316 & 17.12 .307 & Rare \\
\hline & Hornsund & $22-08$ & Hornsund A & 77.0 .470 & 15.35 .374 & $\begin{array}{l}\text { Tourist (av: 200, } \\
\text { max 1876), } \\
\text { research, fishing }\end{array}$ \\
\hline & & $22-08$ & Hornsund B & 77.0 .163 & 15.32 .970 & $\begin{array}{l}\text { Tourist (av: } 200, \\
\text { max 1876), } \\
\text { research, fishing }\end{array}$ \\
\hline & & $22-08$ & Hornsund C & 76.59 .915 & 15.31 .766 & $\begin{array}{l}\text { Tourist (av: 200, } \\
\text { max 1876), } \\
\text { research, fishing }\end{array}$ \\
\hline & \begin{tabular}{|l|} 
Bellsund \\
- Calypso \\
Bay
\end{tabular} & 24-08 & Bellsund $A$ & 77.33 .469 & 14.31 .390 & $\begin{array}{l}\text { Tourist (av. 70, } \\
\text { max 882), } \\
\text { research }\end{array}$ \\
\hline & & 24-08 & Bellsund B & 77.31 .996 & 14.46 .278 & $\begin{array}{l}\text { Tourist (av. 70, } \\
\text { max 882), } \\
\text { research }\end{array}$ \\
\hline & Isfjorden & $26-08$ & Longyearbyen A & 77.13 .33 & 15.17 .36 & $\begin{array}{l}\text { Residential, } \\
\text { tourist, fishing }\end{array}$ \\
\hline
\end{tabular}




\begin{tabular}{|c|c|c|c|c|c|c|}
\hline & & $26-08$ & Longyearbyen B & 78.13 .46 & 15.35 .38 & \begin{tabular}{|l} 
Residential, \\
tourist, fishing
\end{tabular} \\
\hline & & 04-09 & Alkepynten & 78.12 .50 & 13.50 .34 & $\begin{array}{l}\text { Tourist (av: } 155 \text {, } \\
\max : 1315 \text { ) }\end{array}$ \\
\hline & $\begin{array}{l}\text { Raudfjord } \\
\text { en }\end{array}$ & $20-08$ & Alicehamna & 79.44 .32 & 12.12 .26 & $\begin{array}{l}\text { Tourist (av: 52, } \\
\text { max: } 931 \text { ), } \\
\text { fishing }\end{array}$ \\
\hline & $\begin{array}{l}\text { Liefdefjor } \\
\text { den }\end{array}$ & $31-08$ & Bockfjorden & 79.27.21 & 13.19 .09 & $\begin{array}{l}\text { Tourist (av: 89, } \\
\operatorname{max:} 1305 \text { ) }\end{array}$ \\
\hline & $\begin{array}{l}\text { Hinlopen } \\
\text { Strait }\end{array}$ & 01-09 & Faksevågen & 79.33 .06 & 17.38 .40 & $\begin{array}{l}\text { Tourist (av: 217, } \\
\text { max: 597) }\end{array}$ \\
\hline UNIS & $\begin{array}{l}\text { Rijpfjorde } \\
n\end{array}$ & $13-08$ & Vindbukta & 80.17 .907 & 22.34 .382 & Research \\
\hline IOPAN & $\begin{array}{l}\text { Prins } \\
\text { Karlforlan } \\
\text { d } 2\end{array}$ & 04-08 & Davidsonpynten & 7821.304 & 1156.784 & Research \\
\hline & $\begin{array}{l}\text { Prins } \\
\text { Karlforlan } \\
\text { d } 3\end{array}$ & 04-08 & Sandbukta & 7815.848 & 1203.637 & Research \\
\hline & \begin{tabular}{|l|} 
Prins \\
Karlforlan \\
d 4
\end{tabular} & 05-08 & Poolepynten & 7826.641 & 1153.747 & $\begin{array}{l}\text { Tourist (av: } 122, \\
\text { max: 360), } \\
\text { research }\end{array}$ \\
\hline & \begin{tabular}{|l|} 
Prins \\
Karlforlan \\
d 5
\end{tabular} & 05-08 & Gordonpynten & 7828.282 & 1134.296 & Research \\
\hline & \begin{tabular}{|l|} 
Prins \\
Karlforlan \\
d 6
\end{tabular} & 06-08 & Ferrierstranda & 7833.926 & 1127.099 & Research \\
\hline & \begin{tabular}{|l|} 
Prins \\
Karlforlan \\
d 7
\end{tabular} & $10-08$ & Mosehjellen & 7852.662 & 1029.983 & Research \\
\hline
\end{tabular}




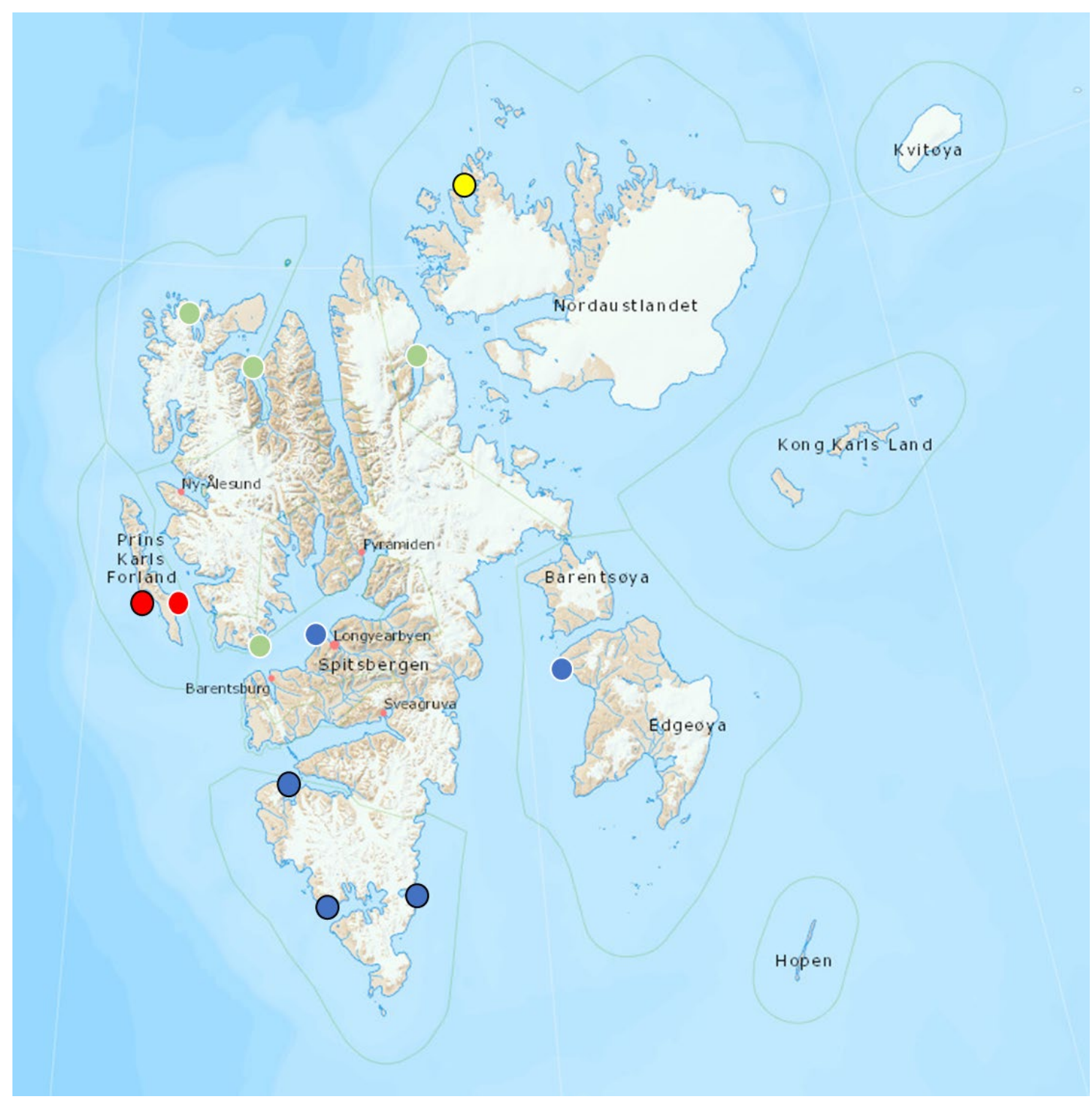

Figure 1 Locations of the sampling campaign in 2018 on Svalbard: red - IOPAN, yellow - UNIS, green and blue - WMR/UNIS; white outline of circle = location frequently visited by tourists, and black outline of circle = locations with less frequent tourist visits. For more detailed maps see Annex 1). Map: www.toposvalbard.npolar.no

\subsection{Beach habitat characteristics}

For each beach, specific habitat characteristics were recorded (Table 2). These were based on quantitative measurements (salinity, temperature), estimates (macroalgae cover, number of debris items per $\mathrm{m}^{2}$ ), comparative impressions (beach slope, exposure), and observations (sediment type, debris type).

Each beach was classified into a beach habitat type based on the visual parameters, morphological and metabarcoding results. Along with beach classification, data on tourist activities and dominant water masses were used to assign a risk level for alien species to each beach. General mitigation strategies will be proposed for high-risk habitats, and monitoring protocols and habitat assessment techniques will be refined. 


\section{Table 2}

Parameters used to characterise beach habitats. Characteristics recorded were site specific and varied according to individual beaches.

\begin{tabular}{|l|l|}
\hline Parameter & Categories and descriptions \\
\hline Beach slope & Gentle, steep, short, variable \\
\hline Potential vectors for alien species & Maritime shipping / tourist shipping / dispersal via anthropogenic (floating) debris \\
\hline Salinity & $\mathrm{mg} / \mathrm{L}, \mathrm{ppt}$ \\
\hline Water temperature & C \\
\hline Beach use & Historical, research, tourist, residential \\
\hline Sediment type & Sand, pebbles, gravel, stones, rock, boulder, ice \\
\hline Exposure & Sheltered, partly exposed, exposed \\
\hline Macroalgae cover & Estimated \% cover \\
\hline Macroalgae distribution & None, patchy, limited to rock \\
\hline Density of natural debris & None, estimated per m ${ }^{2}$, along water line, washed up, live/dead. \\
\hline Natural debris type & Logs, kelp, fucus, barnacles \\
\hline Amount Anthropogenic debris & Number found \\
\hline Type anthropogenic debris & Description \\
\hline
\end{tabular}

\subsection{Sample collection}

For each beach, soft and hard substrate samples were collected to assess dominant and alien marine species living on the beach, using both morphological identification techniques (IOPAN, Poland; UNIS/Akvaplan-niva, Norway) and innovative metabarcoding techniques (Wageningen University, the Netherlands) (Table 3, see Annex 2 for sampling protocol).

The sampling consisted of two samples per transect (low tide, mid tide and high tide) and two transects per beach $(T 1, T 2)$. For the beaches that could only be sampled at the low tide line due to circumstances, four samples were then collected along the water line (T1, T2, T3, T4). See Figure 2 and Figure 3 for the numbers of collected samples per beach for soft and hard substrate.

Hard and soft substrate samples, as well as debris samples for metabarcoding analysis were directly placed in a container or plastic zip-lock and stored frozen or preserved in $>97 \%$ ethanol until extraction and analysis at the WMR lab (the Netherlands).

\section{Table 3}

Type of samples collected at each beach. Samples were subject to change according to what was possible at each location.

\begin{tabular}{|c|c|c|c|}
\hline Sample type & Sample method & Number of samples & Analysis \\
\hline $\begin{array}{l}\text { Soft sediment - } \\
\text { macrofauna }\end{array}$ & $10 \mathrm{~cm} \varnothing$ core, $5-10 \mathrm{~cm}$ deep & $\begin{array}{l}3 \text { replicates at low and mid tide mark where } \\
\text { possible }\end{array}$ & $\begin{array}{l}\text { Morphological identification } \\
\text { Metabarcoding }\end{array}$ \\
\hline $\begin{array}{l}\text { Soft sediment - } \\
\text { meiofauna }\end{array}$ & $3.6 \mathrm{~cm} \varnothing$ core, $5-10 \mathrm{~cm}$ deep & $\begin{array}{l}3 \text { replicates at low and mid tide mark where } \\
\text { possible }\end{array}$ & $\begin{array}{l}\text { Morphological identification } \\
\text { Metabarcoding } \\
\text { Sediment grain size }\end{array}$ \\
\hline Hard substrate & $25 \times 25 \mathrm{~cm}^{2}$ scraping & $\begin{array}{l}3 \text { replicates at low and mid tide mark where } \\
\text { possible }\end{array}$ & $\begin{array}{l}\text { Morphological identification } \\
\text { Metabarcoding }\end{array}$ \\
\hline Debris & $\begin{array}{l}10 \text { min collection along } \\
\text { transect }\end{array}$ & $\begin{array}{l}\text { One } 10 \text { min transect at low and mid tide } \\
\text { mark where possible. }\end{array}$ & $\begin{array}{l}\text { Morphological identification } \\
\text { Metabarcoding }\end{array}$ \\
\hline
\end{tabular}




\subsection{Soft substrate (sediment) samples}

Soft substrate samples for macrofauna analysis were collected using a $10 \mathrm{~cm}$ diameter core $10 \mathrm{~cm}$ deep at three tidal levels (low tide, mid tide and high tide) where possible (Figure 2). The sample was sieved through a $1 \mathrm{~mm}$ mesh on site using ambient seawater and stored in $4 \%$ buffered formaldehyde (with Bengal rose) in plastic sample tubes or jars until further morphological identification of macroorganisms in the Akvaplan-niva lab.

For meiofauna analysis, unsieved sediment samples were directly placed into a plastic jar. Samples were taken with a $3.6 \mathrm{~cm}$ core $10 \mathrm{~cm}$ deep from each of the three tidal heights. The samples were preserved in $4 \%$ buffered formaldehyde (with Bengal rose).

For metabarcoding analysis, a plastic $50-\mathrm{ml}$ tube was filled with the upper $1 \mathrm{~cm}$ of the sediment at each of the three tidal heights. The tubes were stored frozen until extraction and analysis at the WMR lab (the Netherlands). These samples were later also used for grain size analysis.
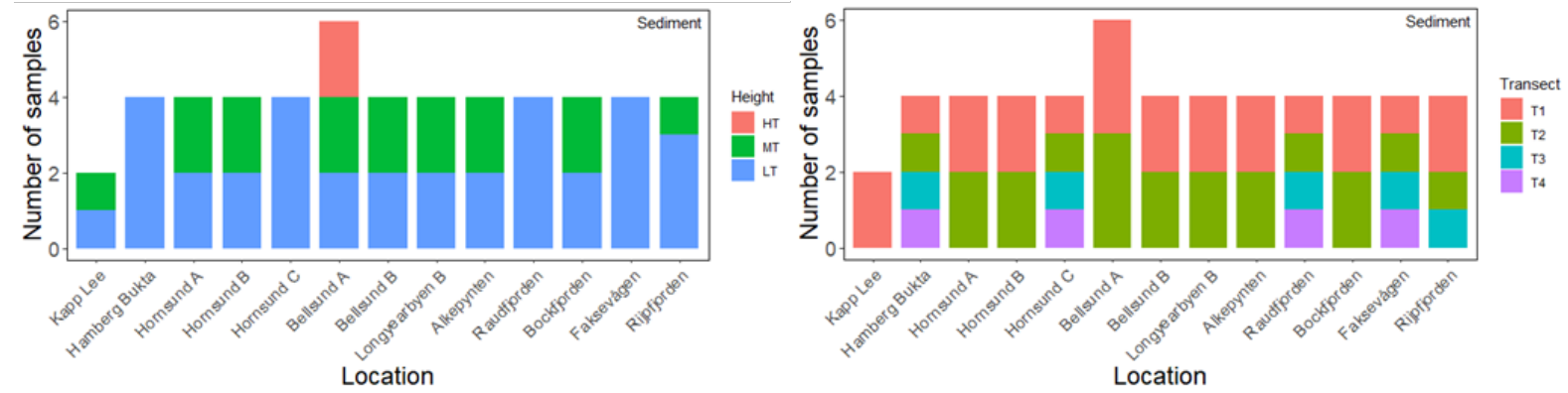

Figure 2 Numbers of soft substrate (sediment) samples collected at the beaches around Svalbard in 2018; per height on the beach (left) and number of transects per beach (right).

\subsection{Hard substrate samples}

Where hard substrate was present, sampling involved scraping the substrate clean with a scalpel within a $25 \times 25 \mathrm{~cm}$ frame, at the tidal heights where possible (Figure 3). Samples were placed into sample tubes or plastic zip-lock bags and placed in $4 \%$ buffered formaldehyde (with Bengal rose) for morphological identification or frozen for eDNA analysis.
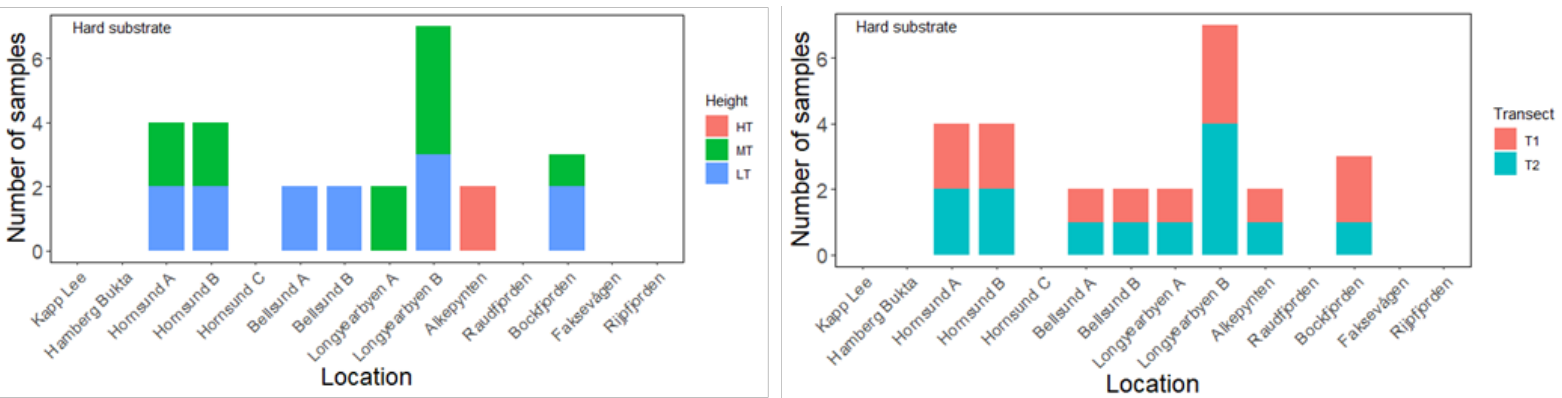

Figure 3 Numbers of hard substrate samples collected at the beaches around Svalbard in 2018, per height on the beach (left) and number of transects per beach (right). 


\subsection{Debris samples}

Debris was collected and analysed from the beaches surveyed by WMR and UNIS. To standardise the survey of debris in 'effort by time', debris was collected and counted at and above the high tide mark during a 10 minute transect across each beach by the three same people. The items were identified and recorded using the OSPAR beach litter classification method (OSPAR, 2010). Plastic and natural debris were sampled for encrusting species. Encrusted species on collected debris were recorded, photographed and a maximum of $5 \times 5 \mathrm{~cm} 2$ surface area of each collected item was collected and frozen for DNA metabarcoding. A selection of the collected debris samples could be processed using metabarcoding techniques (see Figure 4).

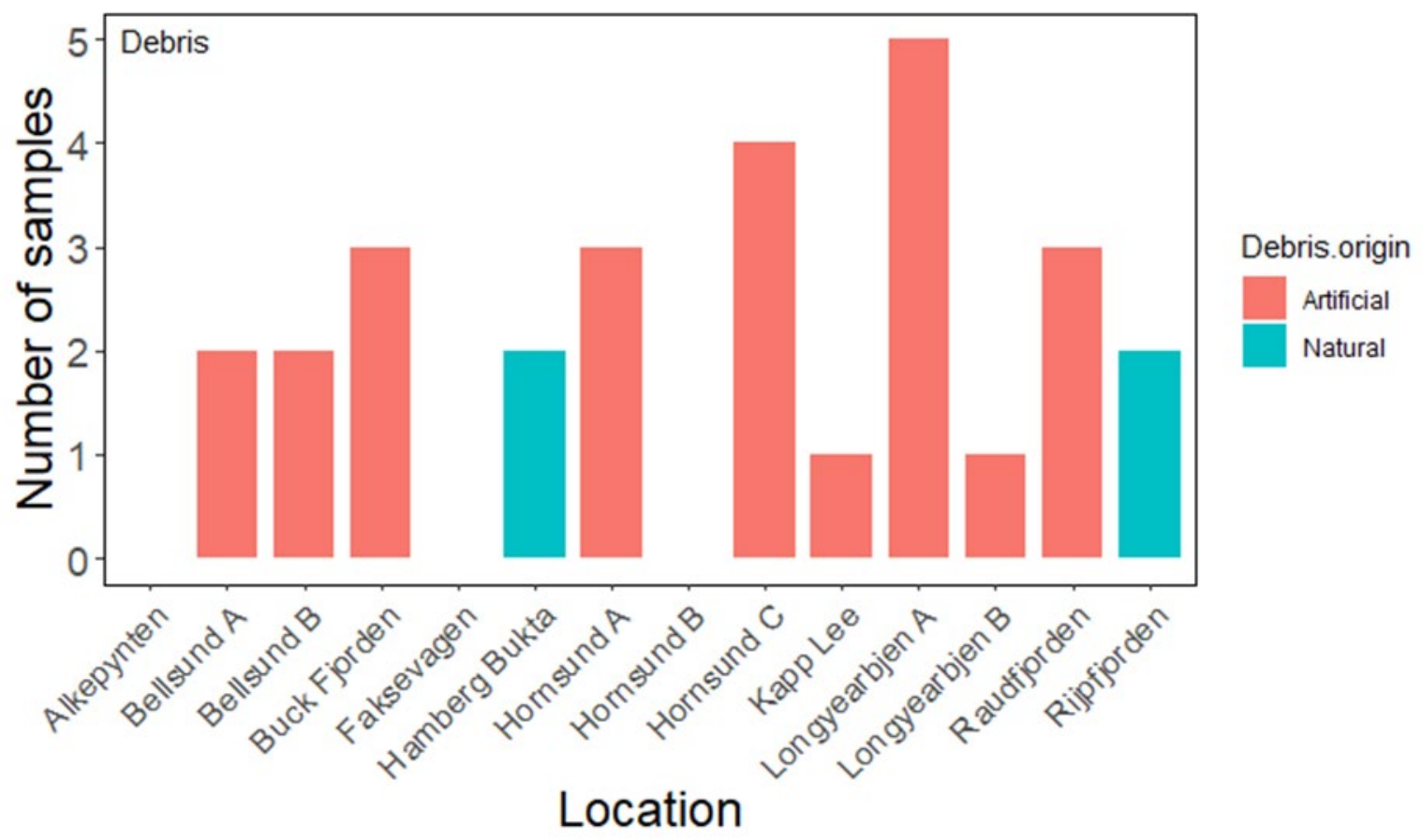

Figure $4 \quad$ Numbers of debris samples collected at the beaches around Svalbard in 2018 that were processed using metabarcoding techniques.

\subsection{Sample analysis and data processing}

\subsubsection{Grain size analysis}

Grain size was analysed by freeze drying the WMR/UNIS collected sediment samples (samples from IOPAN and UNIS were not available for grain size analysis). The samples were then separated through stacked sediment sieves ( $<1 \mathrm{~mm}, 1-2 \mathrm{~mm}, 2-8 \mathrm{~mm},>8 \mathrm{~mm}$ ) on a mechanical sieve shaker. Each size range of sediment was weighed separately to produce an average relative proportion of sediment grain size for each beach. The average proportion of grain size for each beach was calculated using all samples on a beach, regardless of height.

\subsubsection{Morphological identification}

The morphological identification of organisms in the soft and hard substrate samples involved identifying specimens to the lowest possible taxonomic level using standard identification keys. Individuals were counted and weighed to calculate biomass following standard procedures.

IOPAN analysed the meiofauna in the soft sediment and the hard substrate samples, whereas Akvaplan-niva analysed the macrofauna species in the soft sediment samples. 


\subsubsection{DNA metabarcoding}

DNA metabarcoding sample analysis consisted of the analysis of DNA markers 18S and C01 (Slijkerman et al., 2017). Statistical analysis of (meta)barcoding was used to relate results to the international DNA libraries (e.g. Genbank, BOLD) to establish occurrence and possibly distribution of Arctic marine alien species around Svalbard. This method was tested in an earlier project funded by the Svalbard Environmental Protection Fund (17/25) and proved successful in the identification of three potential marine alien species in Kongsfjorden sediment (Van den Heuvel-Greve et al., 2021).

\subsubsection{Data processing}

Community analysis and comparisons were conducted using standard statistical methodology. To determine if species composition changed between sampling locations, sediment types or different heights on the beach at which the samples were collected, a Bray Curtis Dissimilarity Index in binary sense was applied to the dataset. Samples collected were analysed using eDNA metabarcoding and showed the presence (1) and absence (0) (binary data) of different species.

\subsubsection{Assigning risk levels to beaches}

Along with beach classification, data on tourist activities and dominant water masses were used to assign a risk level for alien species to each beach. General mitigation strategies will be proposed for high-risk habitats, and monitoring protocols and habitat assessment techniques will be refined. 


\section{Results and discussion}

\subsection{Beach habitat characteristics}

\subsubsection{Beach descriptions}

The beaches surveyed varied in habitat characteristics, orientation and exposure. Each beach had unique physical attributes relative to the other beaches, even those geographically close by (Table 4).

The beaches had similar salinity (26.9-32.4 ppt). Water temperature ranged from $2.5^{\circ} \mathrm{C}$ at Hornsund $(A)$ to $6.0^{\circ} \mathrm{C}$ at Longyearbyen $(A)$. While most beaches had a gentle or gradual slope, the beaches at Hamberg Bukta, Bellsund B, Raudfjord and Alkepynten were short and steep. Temperature and salinity values in the shallowest water on the beach is extremely changeable and strongly influenced by air temperature. Both may change substantially change within hours. It is therefore recommended to use general Sea Surface Temperature and Salinity for the Svalbard region. This will show "warm" versus "cold" and "marine" versus "brackish" water, hereby forming a more reliable way than a momentary measurement.

All beaches, regardless of whether they were sheltered or exposed had a wide range of observed sediment types from sand to boulders, except Longyearbyen B which was relatively uniformly gravel (Table 5).

Macroalgae appeared to be only present on rocks at Longyearbyen ( $A$ and $B$ ), Bockfjorden and Alkepynten, and subtidally at Bellsund (A and B). Natural debris (predominantly washed up macroalgae) was found along the high tide water line on all beaches except those at Longyearbyen. Felled logs were commonly washed up on Faksevågen (Table 6). Tourists were likely to visit most beaches at some point, and those beaches closed to tourists were visited by researchers. Hamberg Bukta was the only beach with no reported human visitation.

\section{Table 4}

Photos of each beach showing the general habitat characteristics.

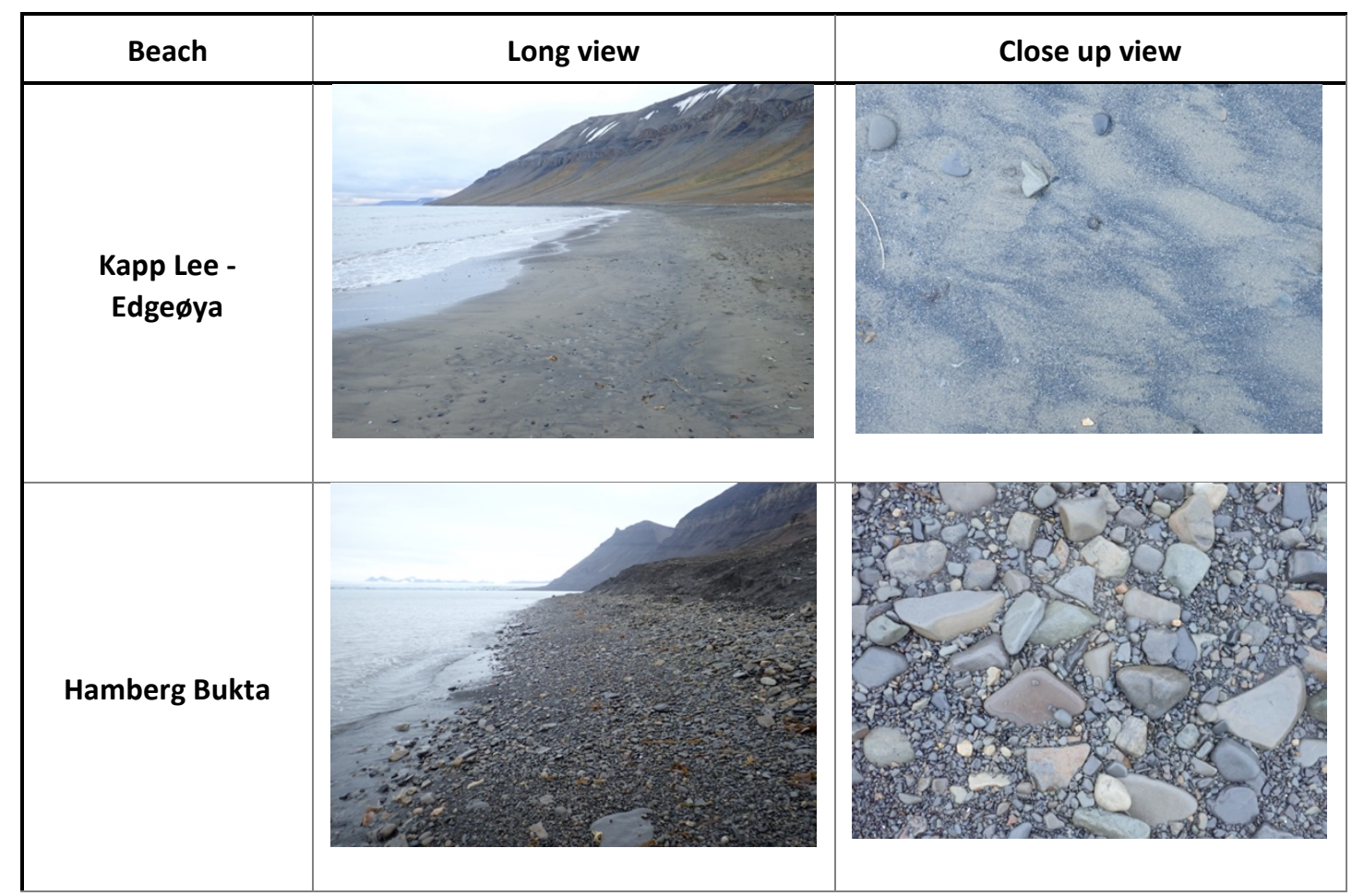




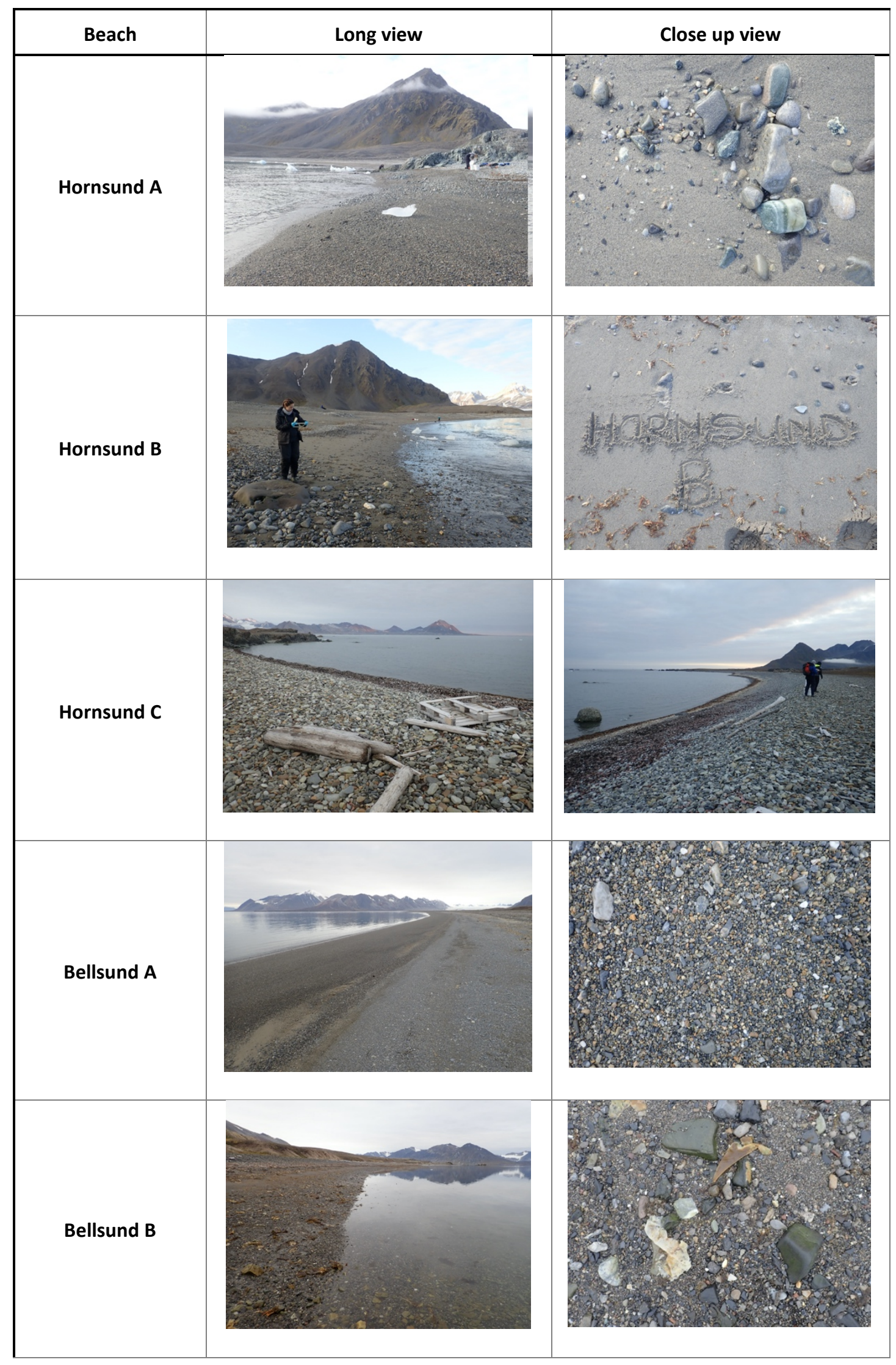




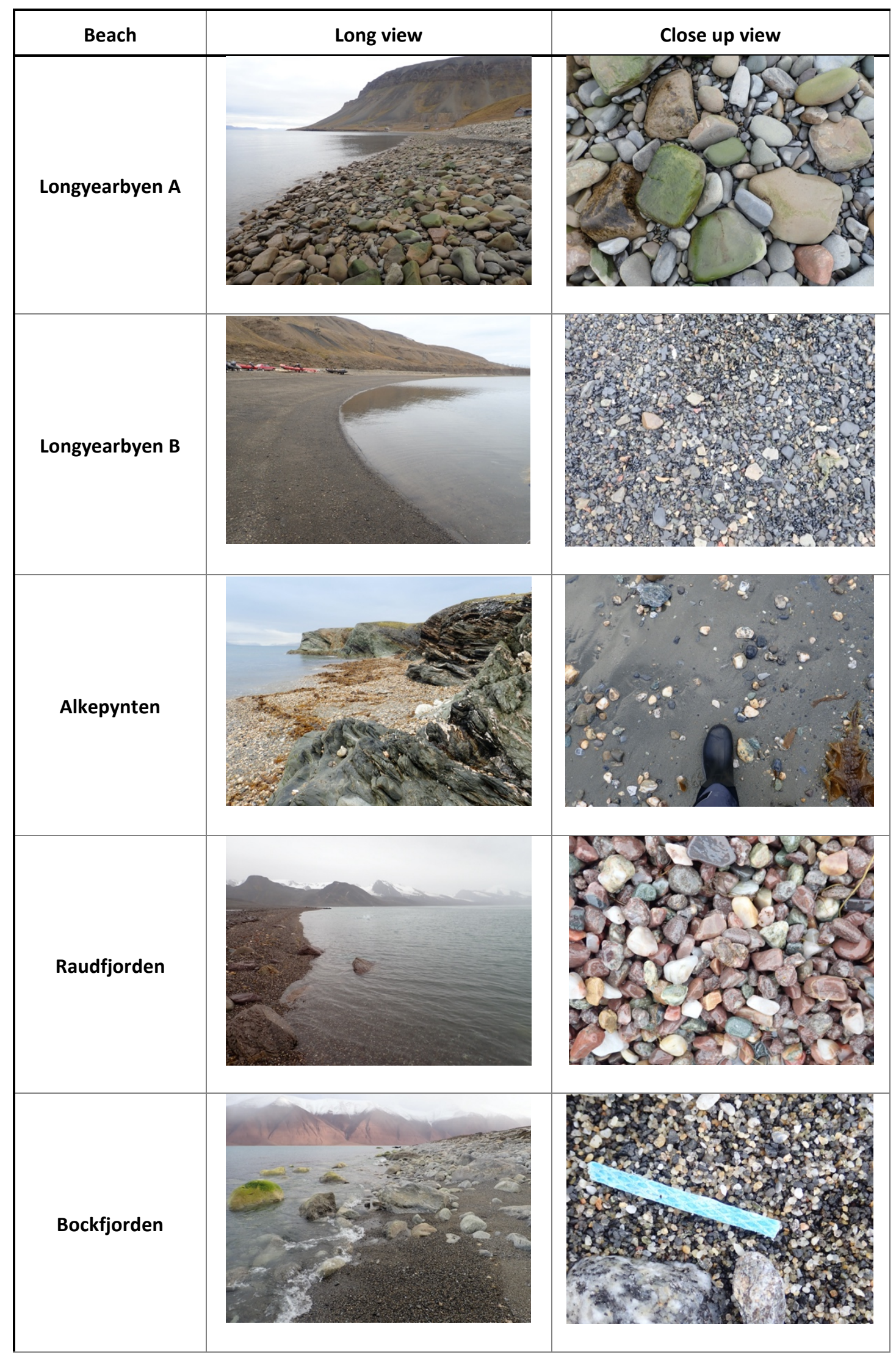




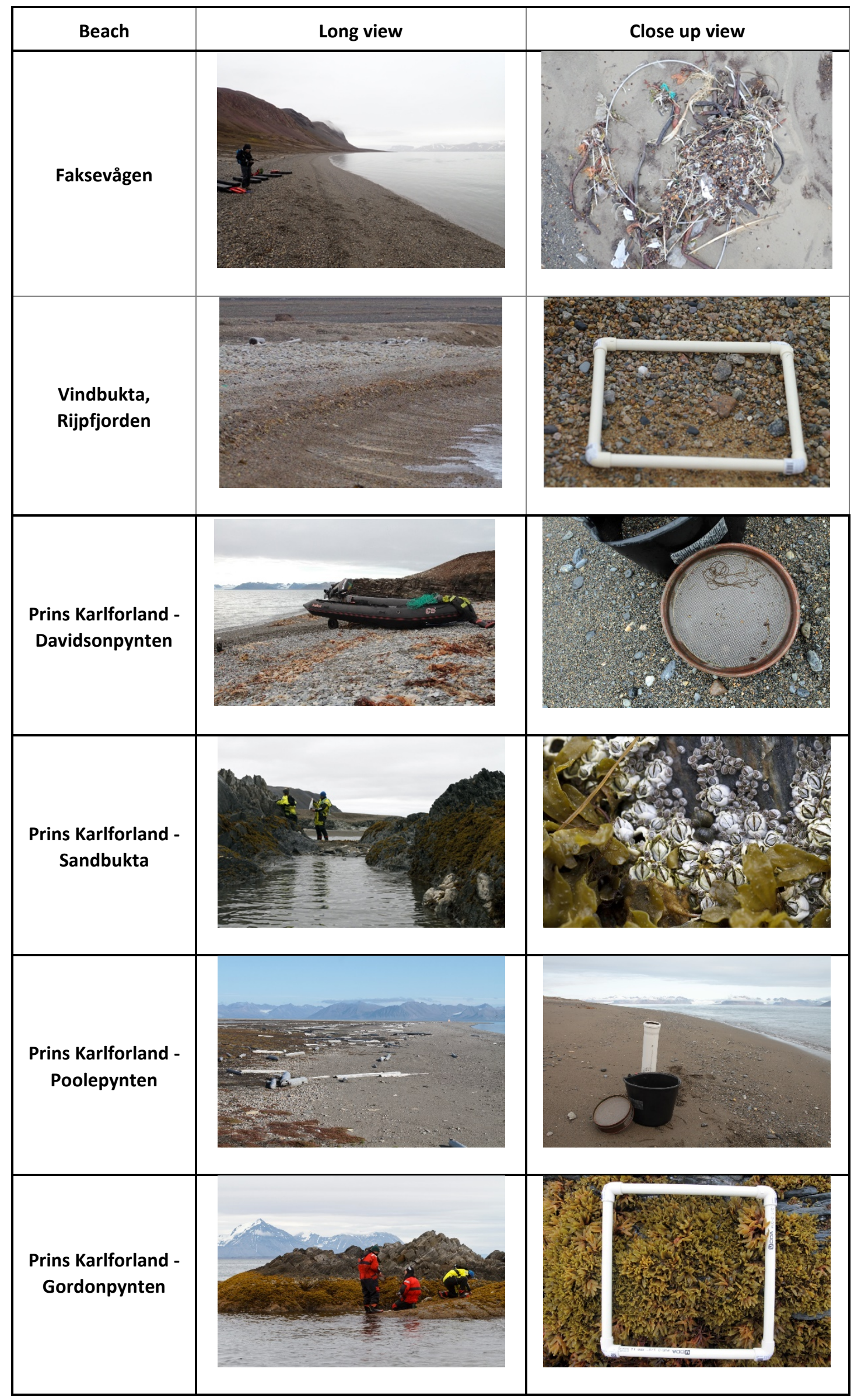




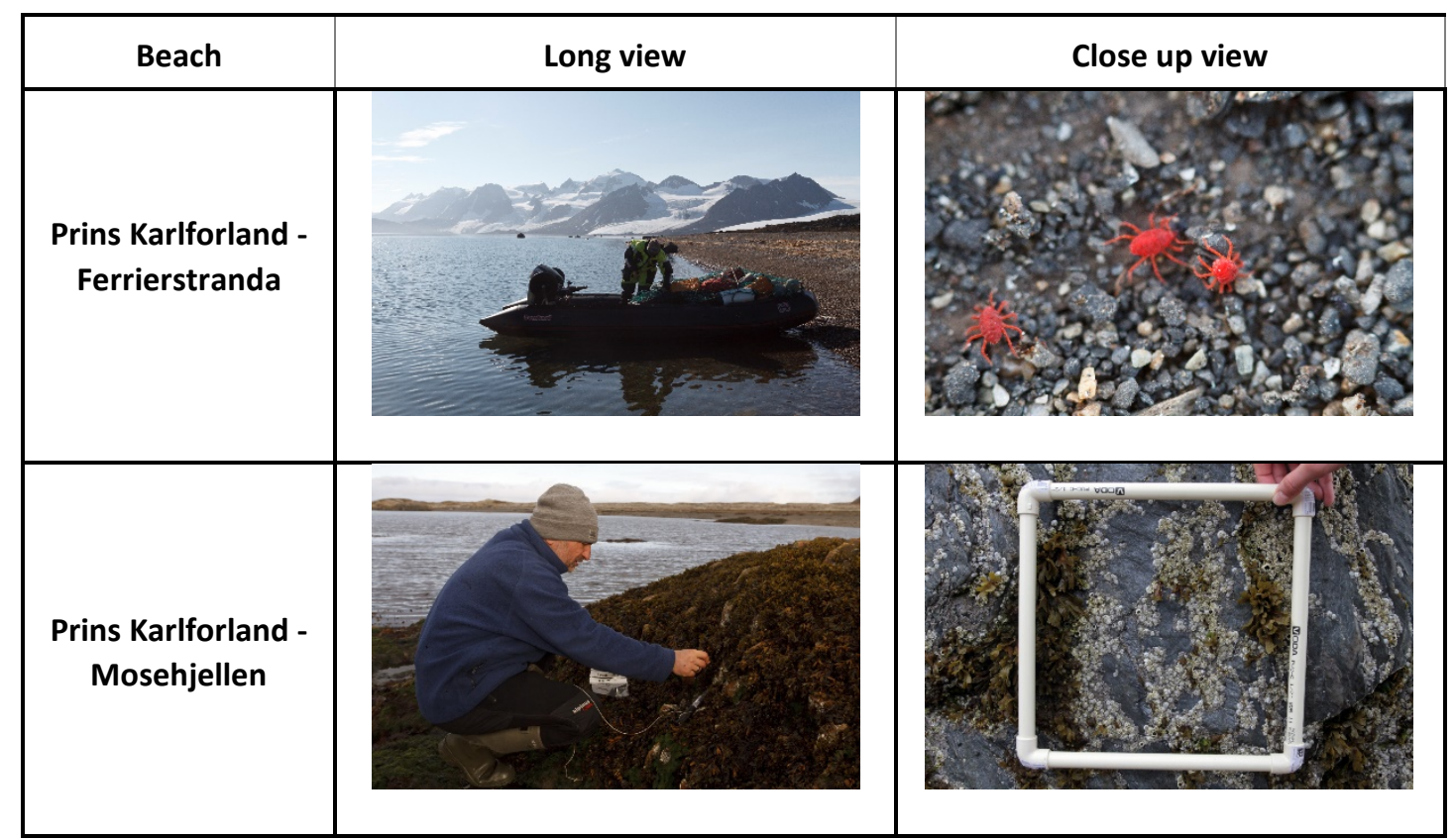

\section{Table 5}

Habitat measurements and observations for the beaches sampled around Svalbard in 2018.

\begin{tabular}{|c|c|c|c|c|c|c|}
\hline Beach & $\begin{array}{l}\text { Beach } \\
\text { slope }\end{array}$ & $\begin{array}{c}\text { Salinity } \\
\text { ppt }\end{array}$ & $\begin{array}{c}\text { Water } \\
\text { temperature } \\
{ }^{\circ} \mathrm{C}\end{array}$ & Type & Exposure & $\begin{array}{c}\text { Exposure } \\
\text { explanation }\end{array}$ \\
\hline $\begin{array}{l}\text { Kapp Lee - } \\
\text { Edgeøya }\end{array}$ & Gentle & 31.8 & 5.4 & $\begin{array}{c}\text { Sand, } \\
\text { pebbles, } \\
\text { gravel }\end{array}$ & $\begin{array}{c}\text { Partly } \\
\text { exposed }\end{array}$ & $\begin{array}{c}\text { Exposed to N, } \\
\text { spit to S }\end{array}$ \\
\hline Hamberg Bukta & $\begin{array}{l}\text { Short, } \\
\text { steep }\end{array}$ & 30.3 & 5.45 & $\begin{array}{c}\text { Pebbles, } \\
\text { stones, } \\
\text { boulders }\end{array}$ & Sheltered & \\
\hline Hornsund A & Gentle & 26.9 & 2.5 & $\begin{array}{c}\text { Sand, } \\
\text { Pebbles, } \\
\text { ice, rock }\end{array}$ & $\begin{array}{c}\text { Partly } \\
\text { exposed }\end{array}$ & $\begin{array}{c}\text { Exposed to } \\
\text { NW }\end{array}$ \\
\hline Hornsund B & Gentle & 26.9 & 2.5 & $\begin{array}{l}\text { Harbour, } \\
\text { sand, } \\
\text { pebbles, } \\
\text { ice }\end{array}$ & Sheltered & \\
\hline Hornsund C & Variable & - & - & $\begin{array}{c}\text { Pebbles, } \\
\text { stones }\end{array}$ & Exposed & \\
\hline Bellsund A & Gentle & 27.9 & 4.35 & $\begin{array}{c}\text { Sand, } \\
\text { pebbles }\end{array}$ & Sheltered & $\begin{array}{c}\text { Entrance to } \\
\text { bay }\end{array}$ \\
\hline Bellsund B & $\begin{array}{l}\text { Short, } \\
\text { steep }\end{array}$ & 27.9 & 4.35 & $\begin{array}{c}\text { Sand, } \\
\text { pebbles, } \\
\text { gravel }\end{array}$ & Sheltered & $\begin{array}{l}\text { Entrance to } \\
\text { bay, in line } \\
\text { with north } \\
\text { west wind }\end{array}$ \\
\hline
\end{tabular}




\begin{tabular}{|c|c|c|c|c|c|c|}
\hline Beach & $\begin{array}{l}\text { Beach } \\
\text { slope }\end{array}$ & $\begin{array}{l}\text { Salinity } \\
\text { ppt }\end{array}$ & $\begin{array}{c}\text { Water } \\
\text { temperature } \\
{ }^{\circ} \mathrm{C}\end{array}$ & Type & Exposure & $\begin{array}{l}\text { Exposure } \\
\text { explanation }\end{array}$ \\
\hline Longyearbyen A & Gradual & 30.5 & 6 & $\begin{array}{c}\text { Stones and } \\
\text { pebbles }\end{array}$ & Exposed & $\begin{array}{l}\text { Open to } \\
\text { Isfjord }\end{array}$ \\
\hline Longyearbyen B & $\begin{array}{l}\text { Gentle- } \\
\text { gradual }\end{array}$ & 31.9 & 5.4 & Gravel & Exposed & $\begin{array}{l}\text { Open to } \\
\text { Isfjord }\end{array}$ \\
\hline Alkepynten & $\begin{array}{l}\text { Gradual- } \\
\text { steep }\end{array}$ & 29.2 & 5.9 & $\begin{array}{l}\text { Sand, } \\
\text { gravel, } \\
\text { stones, } \\
\text { rocks }\end{array}$ & Exposed & $\begin{array}{l}\text { Open to } \\
\text { Isfjord }\end{array}$ \\
\hline Raudfjord & Steep & 30.6 & 5.2 & $\begin{array}{l}\text { Pebbles, } \\
\text { stones, } \\
\text { rocks }\end{array}$ & $\begin{array}{c}\text { Partly } \\
\text { exposed }\end{array}$ & $\begin{array}{l}\text { In fjord, open } \\
\text { to Arctic sea }\end{array}$ \\
\hline Bockfjorden & Gentle & 32.4 & 5.3 & $\begin{array}{c}\text { Sand, } \\
\text { gravel, } \\
\text { stones, } \\
\text { rocks }\end{array}$ & & \\
\hline Faksevågen & Gentle & 32.3 & 4.6 & $\begin{array}{l}\text { Gravel, } \\
\text { pebbles, } \\
\text { stones }\end{array}$ & $\begin{array}{c}\text { Partly } \\
\text { exposed }\end{array}$ & \\
\hline Vindbukta & Gentle & - & $5.6-$ & $\begin{array}{l}\text { Sand, } \\
\text { pebbels, } \\
\text { stones }\end{array}$ & Exposed & $\begin{array}{c}\text { In fjord, long } \\
\text { westward } \\
\text { fetch }\end{array}$ \\
\hline $\begin{array}{l}\text { Prins Karlforland } \\
\text { Davidsonpynten }\end{array}$ & Gentle & - & - & $\begin{array}{l}\text { Gravel, } \\
\text { pebbles, } \\
\text { stones }\end{array}$ & Exposed & $\begin{array}{l}\text { Open to } \\
\text { Forlandsundet }\end{array}$ \\
\hline $\begin{array}{l}\text { Prins Karlforland } \\
\text { - Sandbukta }\end{array}$ & - & - & - & $\begin{array}{l}\text { Rocky } \\
\text { shore }\end{array}$ & Exposed & $\begin{array}{l}\text { Open to } \\
\text { Forlandsundet }\end{array}$ \\
\hline $\begin{array}{l}\text { Prins Karlforland } \\
\text { - Poolepynten }\end{array}$ & Gentle & - & - & $\begin{array}{l}\text { Sand, } \\
\text { pebbles, } \\
\text { gravel }\end{array}$ & Exposed & $\begin{array}{l}\text { Open to } \\
\text { Forlandsundet }\end{array}$ \\
\hline $\begin{array}{l}\text { Prins Karlforland } \\
\text { - Gordonpynten }\end{array}$ & - & - & - & $\begin{array}{l}\text { Rocky } \\
\text { shore }\end{array}$ & Exposed & $\begin{array}{c}\text { Open to } \\
\text { Forlandsundet }\end{array}$ \\
\hline $\begin{array}{l}\text { Prins Karlforland } \\
\text { - Ferrierstranda }\end{array}$ & Steep & - & - & $\begin{array}{l}\text { Gravel, } \\
\text { pebbles, } \\
\text { stones }\end{array}$ & Exposed & $\begin{array}{l}\text { Open to } \\
\text { Forlandsundet }\end{array}$ \\
\hline $\begin{array}{l}\text { Prins Karlforland } \\
\text { - Mosehjellen }\end{array}$ & - & - & - & $\begin{array}{l}\text { Rocky } \\
\text { shore }\end{array}$ & $\begin{array}{c}\text { Partly } \\
\text { exposed }\end{array}$ & $\begin{array}{l}\text { Open to } \\
\text { Forlandsundet }\end{array}$ \\
\hline
\end{tabular}




\section{Table 6}

Habitat observations for the sample beached around Svalbard in 2018. Numbers given for tourism indicate the average number of visitors to the general region per year between 1996-2010 and the maximum number of visitors per year during that time (data from Governor of Svalbard).

\begin{tabular}{|c|c|c|c|c|c|c|}
\hline Beach & $\begin{array}{c}\text { Macroalgae } \\
\% \text { cover }\end{array}$ & $\begin{array}{l}\text { Macroalgae } \\
\text { distribution }\end{array}$ & $\begin{array}{c}\text { Density of } \\
\text { natural } \\
\text { debris }\end{array}$ & $\begin{array}{c}\text { Natural } \\
\text { debris } \\
\text { type }\end{array}$ & $\begin{array}{c}\text { Suggested } \\
\text { potential } \\
\text { vectors for } \\
\text { alien species }\end{array}$ & $\begin{array}{c}\text { General } \\
\text { observations }\end{array}$ \\
\hline $\begin{array}{l}\text { Kapp Lee - } \\
\text { Edgeøya }\end{array}$ & 0 & & $>20$ & $\begin{array}{l}\text { Old drift } \\
\text { logs }\end{array}$ & $\begin{array}{c}\text { Tourism (av: } \\
\text { 428, max: } \\
2008 \text { ) }\end{array}$ & $\begin{array}{l}\text { Historical } \\
\text { research huts. } \\
\text { Lots of bones. } \\
\text { Walruses. }\end{array}$ \\
\hline Hamberg Bukta & $<1$ & Patchy & 2 per $\mathrm{m}^{2}$ & Kelp & & $\begin{array}{c}\text { Edge of glacial } \\
\text { waters. Beach } \\
\text { too narrow for } \\
\text { transects, so } 4 \\
\text { points along } \\
\text { beach } 10 \mathrm{~m} \\
\text { apart were } \\
\text { sampled }\end{array}$ \\
\hline Hornsund A & 0 & $\begin{array}{c}\text { Patchy, } \\
\text { along water } \\
\text { line }\end{array}$ & $\begin{array}{c}\text { All along } \\
\text { water line }\end{array}$ & Kelp & $\begin{array}{l}\text { Atlantic } \\
\text { current, } \\
\text { fishing, } \\
\text { tourism (av: } \\
\text { 200, max } \\
\text { 1876), } \\
\text { research }\end{array}$ & $\begin{array}{l}\text { Rocks covered } \\
\text { in green } \\
\text { filamentous } \\
\text { algae }\end{array}$ \\
\hline Hornsund B & $<1$ & $\begin{array}{l}\text { Patchy on } \\
\text { rocks }\end{array}$ & $\begin{array}{c}\text { All along } \\
\text { water line }\end{array}$ & Kelp & $\begin{array}{l}\text { Atlantic } \\
\text { current, } \\
\text { fishing, } \\
\text { tourism (av: } \\
\text { 200, max } \\
\text { 1876), } \\
\text { research }\end{array}$ & $\begin{array}{c}\text { Beach } \\
\text { travelled a lot } \\
\text { by researchers } \\
\text { from the } \\
\text { adjacent } \\
\text { station }\end{array}$ \\
\hline Hornsund C & 0 & - & $\begin{array}{c}\text { All along } \\
\text { water line }\end{array}$ & Kelp & $\begin{array}{l}\text { Atlantic } \\
\text { current, } \\
\text { fishing, } \\
\text { tourism (av: } \\
\text { 200, max } \\
\text { 1876), } \\
\text { research }\end{array}$ & $\begin{array}{l}\text { A lot of small } \\
\text { debris - only } \\
100 \mathrm{~m} \text { walked } \\
\text { in } 10 \text { min }\end{array}$ \\
\hline Bellsund A & $\begin{array}{c}\text { Some } \\
\text { subtidal }\end{array}$ & & $\begin{array}{l}\text { Washed } \\
\text { up. Some } \\
\text { live }\end{array}$ & Kelp & $\begin{array}{l}\text { Atlantic } \\
\text { current, } \\
\text { fishing, } \\
\text { tourism (av. } \\
\text { 70, max 882), } \\
\text { research }\end{array}$ & \\
\hline Bellsund B & $\begin{array}{c}\text { Subtidal } \\
\text { only }\end{array}$ & & $\begin{array}{l}\text { Washed } \\
\text { up. Some } \\
\text { live }\end{array}$ & Kelp, fucus & $\begin{array}{l}\text { Atlantic } \\
\text { current, } \\
\text { fishing, } \\
\text { tourism (av. } \\
\text { 70, max 882), } \\
\text { research }\end{array}$ & \\
\hline
\end{tabular}




\begin{tabular}{|c|c|c|c|c|c|c|}
\hline Beach & $\begin{array}{c}\text { Macroalgae } \\
\text { \% cover }\end{array}$ & $\begin{array}{l}\text { Macroalgae } \\
\text { distribution }\end{array}$ & $\begin{array}{c}\text { Density of } \\
\text { natural } \\
\text { debris }\end{array}$ & $\begin{array}{l}\text { Natural } \\
\text { debris } \\
\text { type }\end{array}$ & $\begin{array}{l}\text { Suggested } \\
\text { potential } \\
\text { vectors for } \\
\text { alien species }\end{array}$ & $\begin{array}{c}\text { General } \\
\text { observations }\end{array}$ \\
\hline Longyearbyen A & $\begin{array}{c}\text { Green } \\
\text { filamentous } \\
\text { on rocks }\end{array}$ & $\begin{array}{l}\text { Only on } \\
\text { rocks, } \\
\text { located } \\
\text { over the } \\
\text { beach }\end{array}$ & 0 & & $\begin{array}{l}\text { In the entry } \\
\text { towards } \\
\text { Isfjord }\end{array}$ & $\begin{array}{c}\text { No soft } \\
\text { substrate. } \\
\text { Gammarids } \\
\text { present under } \\
\text { the rocks }\end{array}$ \\
\hline Longyearbyen B & $\begin{array}{l}\text { Only on } \\
\text { half } \\
\text { submerged } \\
\text { rocks }\end{array}$ & $\begin{array}{l}\text { Only on } \\
\text { rocks on } \\
\text { edge of } \\
\text { beach }\end{array}$ & 0 & Fucus & $\begin{array}{l}\text { A sheltered } \\
\text { beach near } \\
\text { the harbour }\end{array}$ & $\begin{array}{l}\text { Some debris } \\
\text { might be land } \\
\text { based. }\end{array}$ \\
\hline Alkepynten & $\begin{array}{l}\text { Up to } 80 \% \\
\text { on rocks }\end{array}$ & $\begin{array}{l}\text { Only on } \\
\text { rocks }\end{array}$ & $\begin{array}{c}\text { All along } \\
\text { water line }\end{array}$ & Kelp & $\begin{array}{c}\text { Tourists (av: } \\
\text { 155, max: } \\
\text { 1315) }\end{array}$ & $\begin{array}{l}\text { Small beach } \\
\text { with strong } \\
\text { tidal influence }\end{array}$ \\
\hline Raudfjord & 0 & 0 & $\begin{array}{c}\text { Some } \\
\text { along } \\
\text { water line }\end{array}$ & Fucus & $\begin{array}{l}\text { Atlantic sea, } \\
\text { Tourism (av: } \\
\text { 52, max: 931), } \\
\text { fishing }\end{array}$ & \\
\hline Bockfjorden & $\begin{array}{l}\text { Only on } \\
\text { half } \\
\text { submerged } \\
\text { rocks }\end{array}$ & $\begin{array}{l}\text { Only on half } \\
\text { submerged } \\
\text { rocks }\end{array}$ & $\begin{array}{c}\text { Some } \\
\text { along } \\
\text { water line }\end{array}$ & $\begin{array}{l}\text { Washed } \\
\text { up kelp }\end{array}$ & $\begin{array}{c}\text { Tourism (av: } \\
\text { 89, max: } \\
1305)\end{array}$ & \\
\hline Faksevågen & 0 & 0 & $\begin{array}{l}\text { Washed up } \\
\text { macroalgae } \\
\text { and logs }\end{array}$ & & $\begin{array}{c}\text { Tourism (av: } \\
\text { 217, max: } \\
597 \text { ) }\end{array}$ & $\begin{array}{c}\text { A lot of fine } \\
\text { debris tangled } \\
\text { in algae }\end{array}$ \\
\hline Vindbukta & $<1$ & $\begin{array}{l}\text { Patches all } \\
\text { over beach }\end{array}$ & $\begin{array}{c}\text { Narrow } \\
\text { bands with } \\
30 \% \text { cover, } \\
\text { overall 5- } \\
10 \%\end{array}$ & $\begin{array}{l}\text { Logs, } \\
\text { algae }\end{array}$ & Research & $\begin{array}{l}\text { Wood } \\
\text { (Siberian } \\
\text { larch), drift } \\
\text { algae, fishing } \\
\text { gear (rope, } \\
\text { floats), crab } \\
\text { pot bait boxes }\end{array}$ \\
\hline $\begin{array}{l}\text { Prins } \\
\text { Karlforland - } \\
\text { Davidsonpynten }\end{array}$ & $<1$ & $\begin{array}{l}\text { Only on half } \\
\text { submerged } \\
\text { rocks }\end{array}$ & $\begin{array}{l}\text { Washed up } \\
\text { macroalgae } \\
\text { and logs }\end{array}$ & Kelp & $\begin{array}{l}\text { Floating } \\
\text { plastic / } \\
\text { currents }\end{array}$ & Plastic litter \\
\hline $\begin{array}{l}\text { Prins } \\
\text { Karlforland - } \\
\text { Sandbukta }\end{array}$ & 70 & $\begin{array}{l}\text { Rocky tidal } \\
\text { shore } \\
\text { Swash zone }\end{array}$ & - & - & $\begin{array}{l}\text { Floating } \\
\text { plastic / } \\
\text { currents }\end{array}$ & Plastic litter \\
\hline $\begin{array}{l}\text { Prins } \\
\text { Karlforland - } \\
\text { Poolepynten }\end{array}$ & 0 & - & Numerous & $\begin{array}{l}\text { Old drift } \\
\text { logs }\end{array}$ & Tourism & $\begin{array}{l}\text { Small plastic } \\
\text { debris }\end{array}$ \\
\hline $\begin{array}{l}\text { Prins } \\
\text { Karlforland - } \\
\text { Gordonpynten }\end{array}$ & 90 & $\begin{array}{l}\text { Rocky tidal } \\
\text { shore } \\
\text { Swash zone }\end{array}$ & - & - & $\begin{array}{l}\text { Floating } \\
\text { plastic / } \\
\text { currents }\end{array}$ & $\begin{array}{l}\text { Small plastic } \\
\text { debris }\end{array}$ \\
\hline
\end{tabular}




\begin{tabular}{|l|c|c|c|c|c|c|}
\hline \multicolumn{1}{|c|}{ Beach } & $\begin{array}{c}\text { Macroalgae } \\
\text { \% cover }\end{array}$ & $\begin{array}{c}\text { Macroalgae } \\
\text { distribution }\end{array}$ & $\begin{array}{c}\text { Density of } \\
\text { natural } \\
\text { debris }\end{array}$ & $\begin{array}{c}\text { Natural } \\
\text { debris } \\
\text { type }\end{array}$ & $\begin{array}{c}\text { Suggested } \\
\text { potential } \\
\text { vectors for } \\
\text { alien species }\end{array}$ & $\begin{array}{c}\text { General } \\
\text { observations }\end{array}$ \\
\hline $\begin{array}{l}\text { Prins } \\
\text { Karlforland - }\end{array}$ & 0 & 0 & $\begin{array}{c}\text { Washed up } \\
\text { macroalgae } \\
\text { and logs }\end{array}$ & Kelp & $\begin{array}{l}\text { Floating } \\
\text { plastic / } \\
\text { currents }\end{array}$ & Plastic litter \\
\hline $\begin{array}{l}\text { Krins } \\
\text { Morlforland - }\end{array}$ & 80 & $\begin{array}{c}\text { Rocky tidal } \\
\text { shore } \\
\text { Swash zone }\end{array}$ & & - & Floating & plastic / \\
currents & Plastic litter \\
\hline
\end{tabular}

\subsubsection{Grain size analysis}

Sediment grain size varied between beaches. Most of the beaches predominantly consisted of grain sizes between $500 \mu \mathrm{m}$ and $2 \mathrm{~mm}$. Sediments from Bellsund A, Faksevågen, Raudfjord, and Rijpfjorden contained predominantly larger grain sizes. Raudfjord in particular was made up only of grain sizes $>1$ $\mathrm{mm}$ and primarily of grain size $>8 \mathrm{~mm}$ (Figure 5 ).

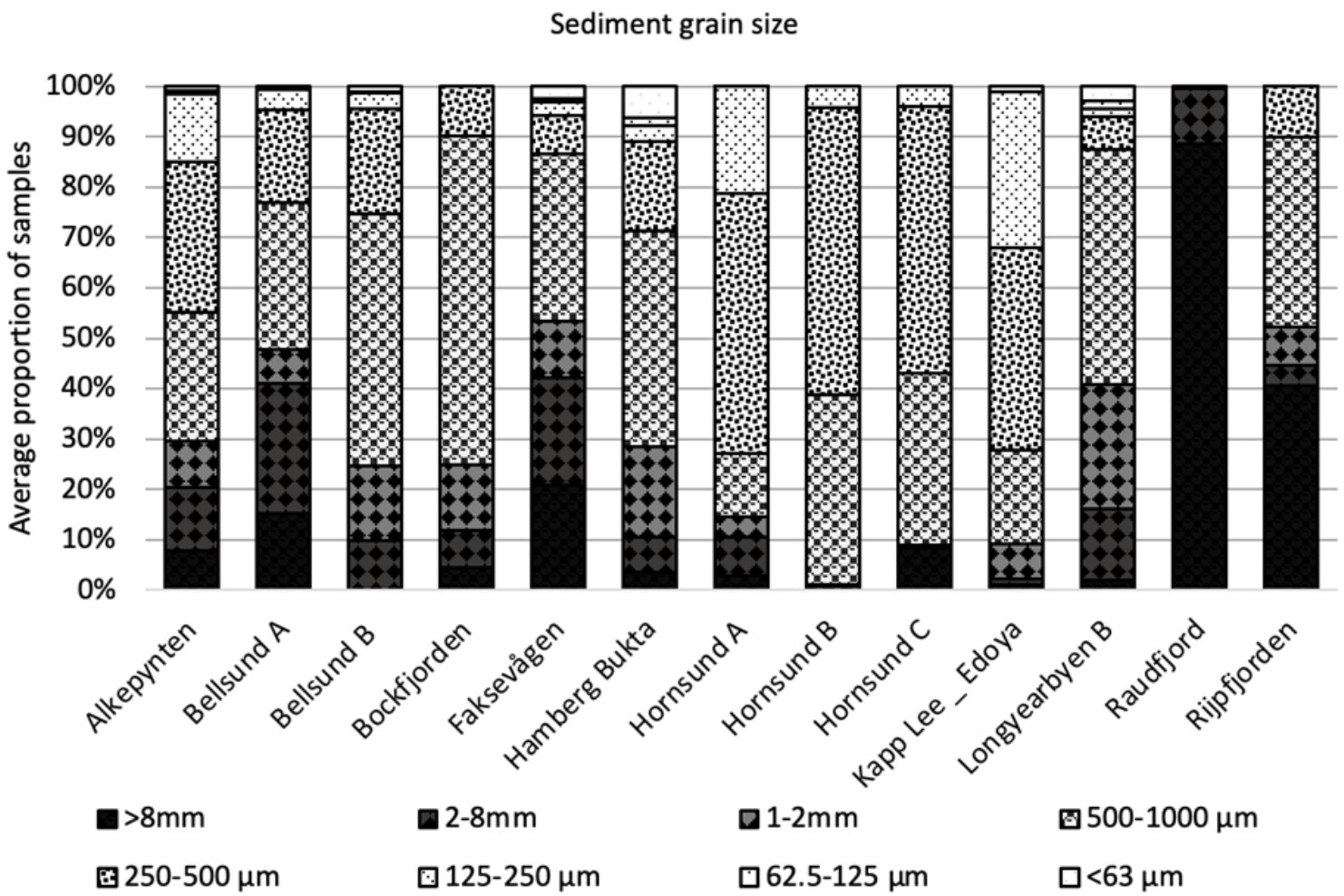

Figure 5 Average relative proportion of different grain sizes in the sediment samples taken by WMR from different beaches around Svalbard. No data were available for Prins Karlsforland. 


\subsection{Soft substrate (sediment)}

\subsubsection{Morphological identification}

Intertidal macrofaunal samples exhibited low apparent diversity, although due to their small size and (for some groups) poorly known taxonomy, identification did not go below family level. Using morphological identification methods for macrofauna, only an annelid and three arthropods were recorded (Table 7). Clearly the most common, and frequently quite abundant, taxon was Oligochaeta. Oligochaetes, though often restricted to meiofaunal samples, were found in 51 of the 52 macrofaunal samples at all tidal levels and in all of the 13 beaches sampled. Other taxa were found sparsely. Terrestrial Collembola (springtails) were found on three beaches and in five samples. Numbers per sample ranged from 1 to 18. A single unidentified coastal Acarina (mite) was found in one sample. Marine calanoid (or unidentified) copepods were found in four macrofaunal samples. Either one or two individuals were recorded, but since these taxa are pelagic, it is likely they were washed in with waves and not sediment residents.

In the intertidal meiofauna samples, the top ten meiofauna species recorded using morphological identification were from the phyla Arthropoda (4), Annelida (2), Turbellaria (1), Nematoda (1) and Bivalvia (1) (Table 8). Abundances were generally 1-20 per core, but reached as high as 255 per sample, with 11 samples having over 40 individuals each. Most common in the samples were the Turbellaria ( $91 \%$ of the samples), followed by the Nematoda ( $86 \%$ of the samples), Copepoda ( $68 \%$ of samples), Oligochaeta (65\%) and Harpacticoida (56\% of samples).

Macrofauna and meiofauna results supported each other, with both identifying Oligochaeta, Collembola, Copepoda and Acari as important phyla. Not all groups found in the meiofauna samples were observed in the macrofauna samples as these samples were sieved, thereby removing most of the organisms smaller than $1 \mathrm{~mm}$.

\section{Table 7}

Most abundant macrofauna with positive identification in the 52 collected soft sediment samples based on morphological identification, Svalbard in 2018.

\begin{tabular}{|c|l|c|c|}
\hline $\begin{array}{l}\text { Lowest taxonomic } \\
\text { level of identification }\end{array}$ & Phylum & $\begin{array}{l}\text { \# samples } \\
\text { identified }\end{array}$ & \%samples \\
\hline Oligochaeta & Annelida & 51 & 98 \\
\hline Collembola & Arthropoda & 5 & 10 \\
\hline Copepoda & Arthropoda & 4 & 7 \\
\hline Acarina & Arthropoda & 1 & 2 \\
\hline
\end{tabular}




\section{Table 8}

Most abundant meiofauna with positive identification in the 57 collected soft sediment samples based on morphological identification, Svalbard in 2018.

\begin{tabular}{|c|l|c|c|}
\hline $\begin{array}{l}\text { Lowest taxonomic level } \\
\text { of identification }\end{array}$ & Phylum & $\begin{array}{l}\text { \# samples } \\
\text { identified }\end{array}$ & \%samples \\
\hline Turbellaria & Turbellaria & 52 & 91 \\
\hline Nematoda & Nematoda & 49 & 86 \\
\hline Copepoda nauplii & Arthropoda & 39 & 68 \\
\hline Oligochaeta & Annelida & 37 & 65 \\
\hline Harpacticoida & Arthropoda & 32 & 56 \\
\hline Collembola & Arthropoda & 19 & 33 \\
\hline Acari & Arthropoda & 19 & 33 \\
\hline Bivalvia & Bivalvia & 18 & 32 \\
\hline Ostracoda & Arthropoda & 3 & 5 \\
\hline Polychaeta & Annelida & 1 & 2 \\
\hline
\end{tabular}

\subsubsection{Metabarcoding}

Based on the metabarcoding technique, a total of 147 species were detected in the soft sediment. The number of species varied between locations with the highest number detected in Hamberg Bukta $(>40)$ and the fewest in Hornsund A and B (<22) (Figure 6 and Figure 7). Species composition was not significantly affected by the height of the beach where the sample was taken (at the tide mark, middle of the beach or top part of the beach).

The species detected belonged to a total of 20 different phyla (Figure 7). The phylum with the largest number of species present in the samples was the Ciliophora (36 species), followed by the Ochrophyta (34 species), Nematoda (11 species) and Myzozoa (10 species).

The top ten species identified using eDNA metabarcoding in the soft sediment samples belong to the phylums Ochrophyta (4), Ciliophora (2), Myzozoa (2), Rhodophyta (1) and Arthropoda (1) (Table 9).

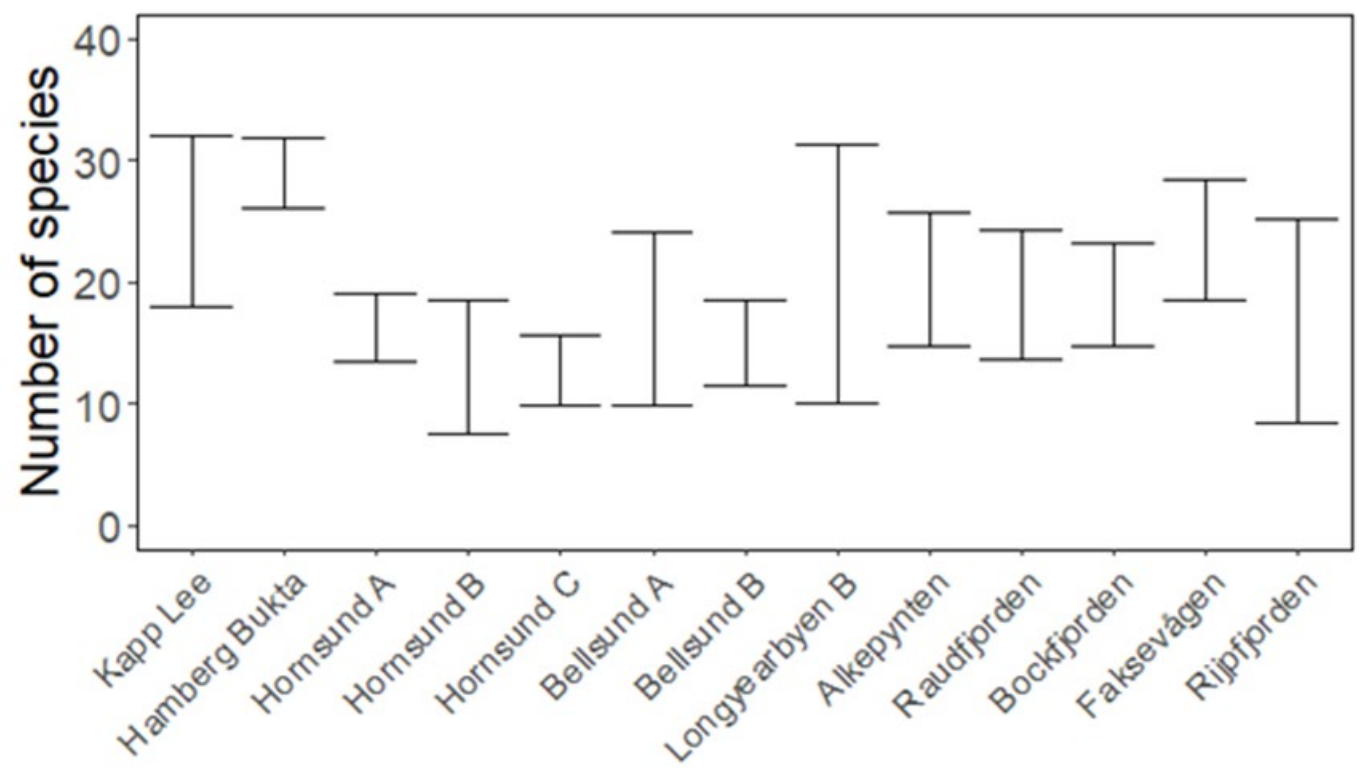

Figure 6 Number of species detected per sample in the soft sediment using eDNA metabarcoding of the beaches sampled around Svalbard in 2018 per location. 


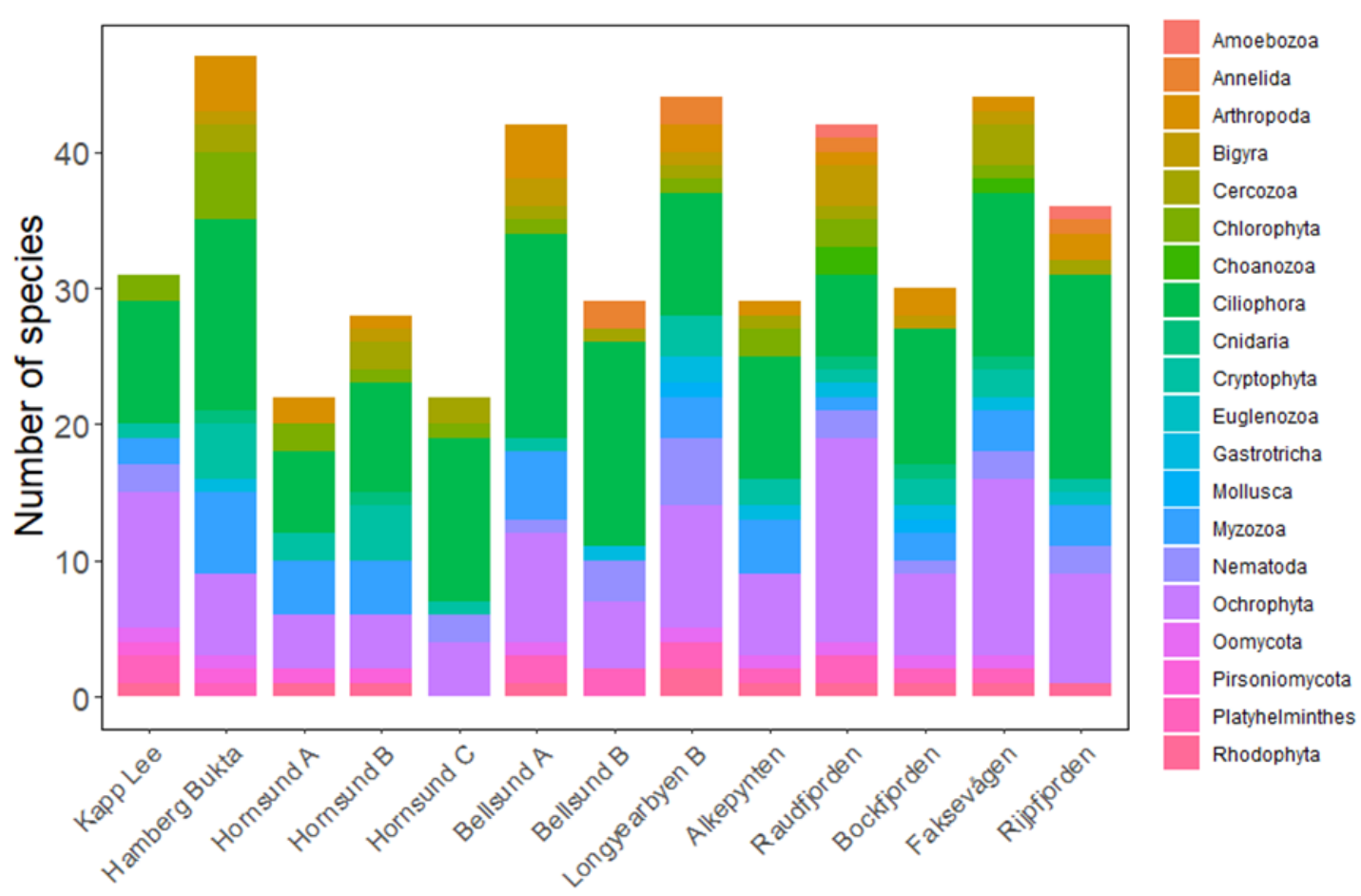

Figure $7 \quad$ Number of species from different phyla and family detected using eDNA metabarcoding in the soft sediment of the beaches sampled around Svalbard in 2018 per location (note that the number of transects and samples differed between locations).

\section{Table 9}

Top ten species with positive identification in the 52 collected sediment samples based on eDNA metabarcoding, Svalbard in 2018.

\begin{tabular}{|l|l|c|c|}
\hline Latin name & Phylum & $\begin{array}{l}\text { \# samples } \\
\text { identified }\end{array}$ & \%samples \\
\hline Paraphysomonas sp. & Ochrophyta & 38 & 73 \\
\hline Pylaiella littoralis & Ochrophyta & 37 & 71 \\
\hline Pyropia sp. & Rhodophyta & 30 & 58 \\
\hline Pseudochilodonopsis fluviatilis & Ciliophora & 30 & 58 \\
\hline Bacillaria sp. & Ochrophyta & 29 & 56 \\
\hline Phialina sp. & Ciliophora & 28 & 54 \\
\hline Gyrodinium helveticum & Myzozoa & 26 & 50 \\
\hline Skeletonema sp. & Ochrophyta & 24 & 46 \\
\hline Aduncodinium glandula & Myzozoa & 24 & 46 \\
\hline Oithona similis & Arthropoda & 23 & 44 \\
\hline
\end{tabular}

\subsubsection{Comparison of morphological techniques for soft substrate}

There was an obvious difference in the results produced by the morphological identification and metabarcoding techniques. The morphological technique primarily identified larger, singular organisms; annelids, arthropods, turbellarians, nematodes and bivalves. The metabarcoding technique identified smaller organisms including algae (Ochrophyta and Rhodophyta), microbes such as (Ciliophora, and Myzozoa), and just a single arthropod. 


\subsection{Hard substrate}

\subsubsection{Morphological identification}

Most of the beaches had limited, or no hard substrate to sample. The hard substrate that could be sampled consisted primarily of rocks/boulders which could be surface scraped. Based on morphological identification the top ten species recorded were from the phyla Ochrophyta (3), Arthropoda (3), Annelida (2), and Mollusca (2) (Table 10).

\section{Table 10}

Top ten organisms with positive identification in the 49 collected hard substrate samples based on morphological identification, Svalbard in 2018.

\begin{tabular}{|l|l|l|l|}
\hline $\begin{array}{l}\text { Lowest taxonomic } \\
\text { level of identification }\end{array}$ & Phylum & $\begin{array}{l}\text { \# } \\
\text { samples } \\
\text { identified }\end{array}$ & \%samples \\
\hline $\begin{array}{l}\text { filamentous algae } \\
\text { Pylaiella/Ectocarpus }\end{array}$ & Ochrophyta & 38 & 78 \\
\hline Fucus distichus & Ochrophyta & 28 & 57 \\
\hline Amphipoda & Arthropoda & 25 & 51 \\
\hline Oligochaeta & Annelida & 23 & 47 \\
\hline Balanus sp. & Arthropoda & 22 & 45 \\
\hline Insecta & Arthropoda & 9 & 18 \\
\hline Gastropoda & Mollusca & 9 & 18 \\
\hline Saccharina latissima & Ochrophyta & 5 & 10 \\
\hline Bivalvia & Mollusca & 5 & 10 \\
\hline Fabricia sp. & Annelida & 5 & 10 \\
\hline
\end{tabular}

\subsubsection{Metabarcoding}

Based on the metabarcoding technique, a total of 71 species were detected in the hard substrate samples. In general, the number of species found per sample was highest in Bellsund $A$ and $B$ and lowest in Longyearbyen $A$ (Figure 8 ). The number of species varied between locations with the highest number detected in Longyearbyen $B(>30)$ and the fewest in Longyearbyen A $(<8)$ (Figure 9). Species composition was not significantly affected by the height of the beach where the sample was taken (at the tide mark, middle of the beach or top part of the beach).

The top ten species that were identified in the hard substrate samples were belonging to the phyla Ochrophyta (8), Ciliophora (1), and Arthropoda (1) (Table 11). 


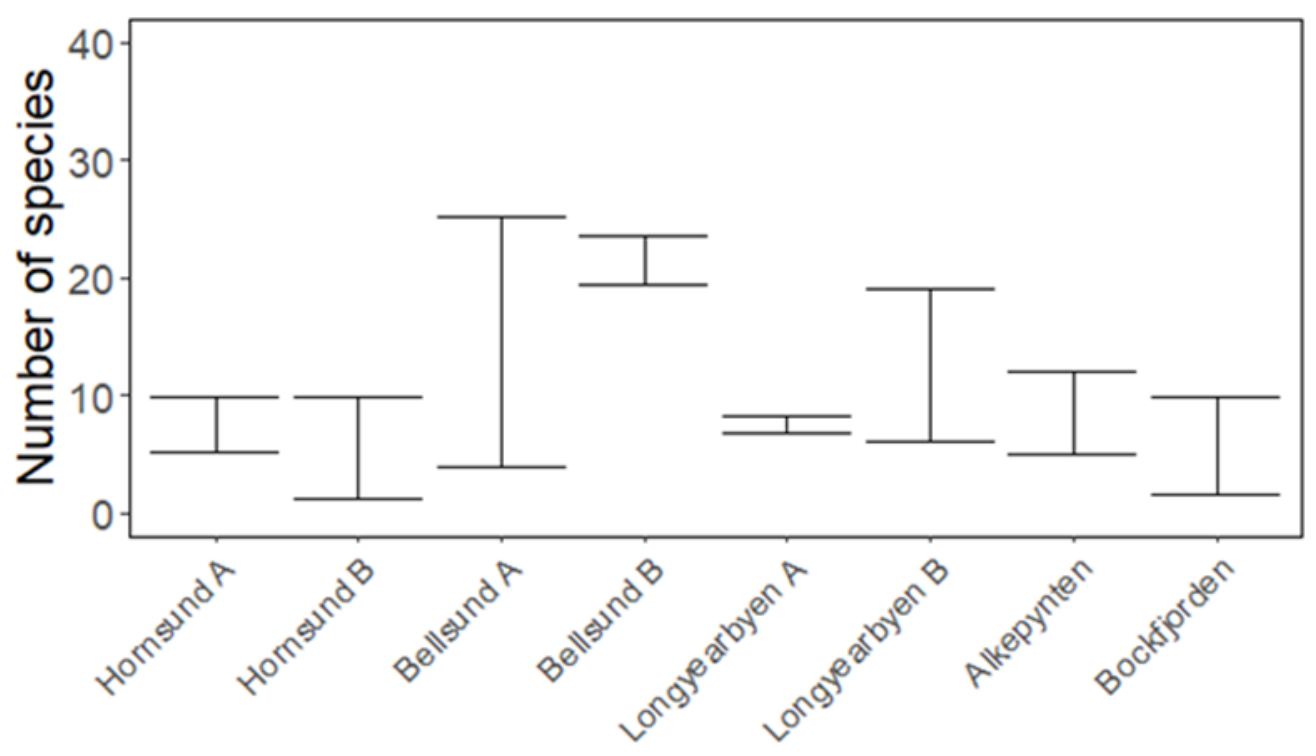

Figure $8 \quad$ Number of species detected using eDNA metabarcoding in the hard substrate from different tidal heights of the beaches sampled around Svalbard in 2018 per location (note that samples depended on the presence of hard substrate).

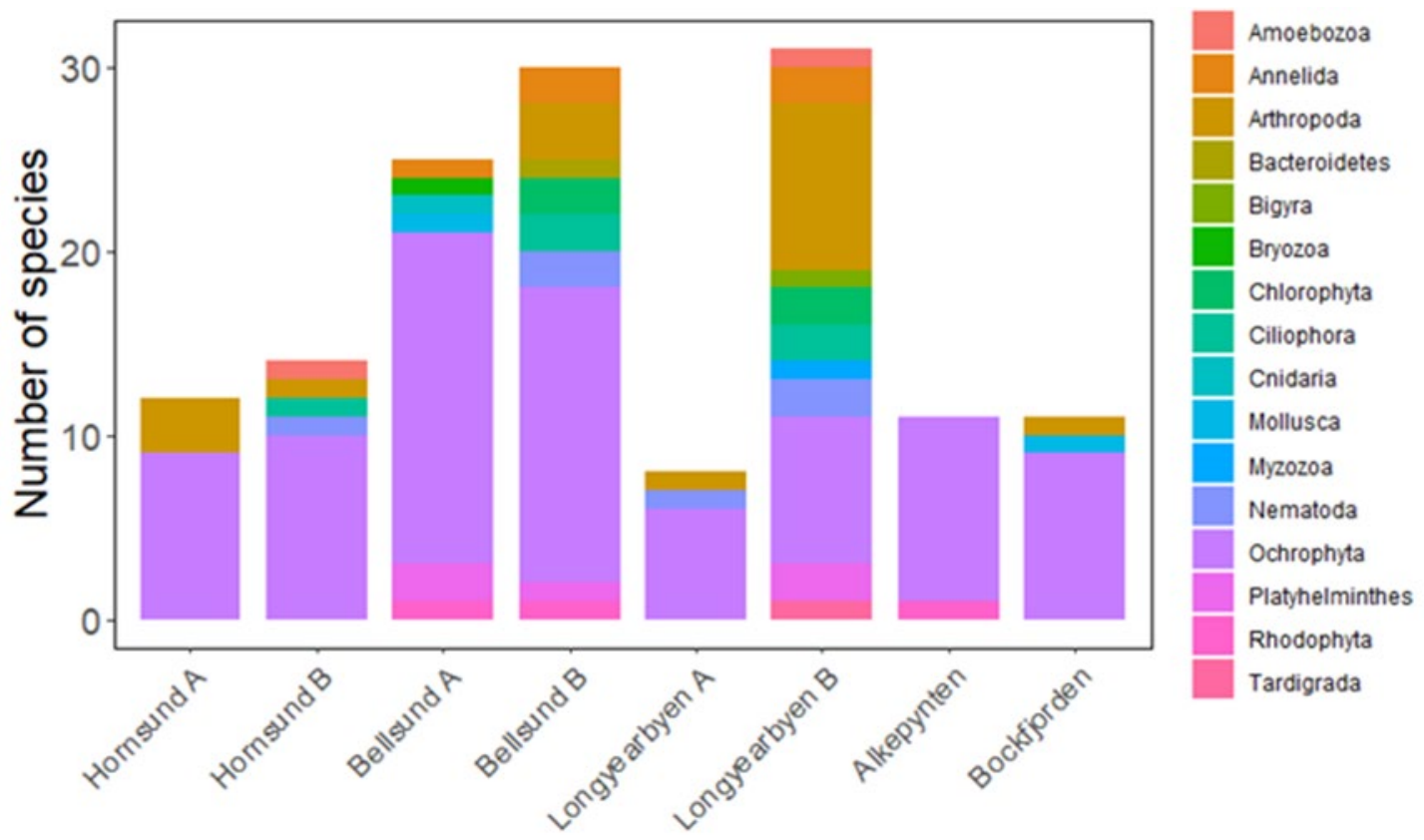

Figure $9 \quad$ Number of species from different phyla and family detected using eDNA metabarcoding in the hard substrate from different transects of the beaches sampled around Svalbard in 2018 per location (note that the number of transects differed between locations that samples depended on the presence of hard substrate). 
Table 11

Top ten species with positive identification in the 26 collected hard substrate samples based on eDNA metabarcoding, Svalbard in 2018.

\begin{tabular}{|l|l|c|c|}
\hline Latin name & Phylum & $\begin{array}{l}\text { \# samples } \\
\text { identified }\end{array}$ & \%samples \\
\hline Licmophora juergensii & Ochrophyta & 19 & 73 \\
\hline Pylaiella littoralis & Ochrophyta & 18 & 69 \\
\hline Fucus sp. & Ochrophyta & 15 & 58 \\
\hline Pylaiella washingtoniensis & Ochrophyta & 13 & 50 \\
\hline Chordaria flagelliformis & Ochrophyta & 13 & 50 \\
\hline Grammonema striatula & Ochrophyta & 10 & 38 \\
\hline Ectocarpus sp. & Ochrophyta & 10 & 38 \\
\hline Dysteria lanceolata & Ciliophora & 10 & 38 \\
\hline Euterpina sp. & Arthropoda & 8 & 31 \\
\hline Tabularia sp. & Ochrophyta & 7 & 27 \\
\hline
\end{tabular}

\subsubsection{Comparison of identification techniques for hard substrate}

There was an obvious difference in the results produced by the morphological identification and metabarcoding techniques for the hard substrate samples. The visual morphological technique primarily identified larger, singular organisms; arthropods, annelids, molluscs and some Ochrophyta. The metabarcoding technique identified smaller organisms; eight species of Ochrophyta, a Ciliophora, and a single arthropod species.

For both the soft and hard substrate samples, the metabarcoding technique produced taxonomic identification at a higher resolution (often identifying to genus or species level), compared with the morphological method. Identification to species level is important because NIS may share a genus with native species and could therefore be missed in identification when using techniques that provide less detailed information.

The use of both morphological identification and DNA metabarcoding together produces complimentary data and a more thorough insight into the biodiversity of the beach than using one method exclusively.

\subsection{Debris}

\subsubsection{Collected debris}

Anthropogenic debris was found on all surveyed beaches, except Alkepynten and Hamberg Bukta. All other beaches had varying amounts of debris, from Kapp Lee, where only a single piece of neoprene was found, to Faksevågen, where 61 items were recorded, and Longyearbyen B, where 49 items were recorded during a 10-minute stroll along the high tide level (Figure 10). At Faksevågen much of the debris was found in the form of small, unidentified plastic pieces entangled in bunches of washed-up kelp and seaweed, while at Longyearbyen $B$ the debris was generally unattached and strewn across the beach. Debris found at Hamberg Bukta and Rijpfjorden were classified as natural debris (kelp and seaweed). At Rijpfjorden anthropogenic debris was also observed at the beach, though not at the high tide and transects that were sampled for this study (Table 6).

There was no, or very little debris found on Storfjord beaches (Kapp Lee and Hamberg Bukta). Hamberg Bukta is not connected to the North Atlantic current and it is not visited by tourists. For these reasons Hamberg Bukta may not be a sink location for litter. 
Alkepynten is a narrow, short beach surrounded by cliffs. Located near the mouth of the Kongsfjorden, it is exposed to high currents. The small size of the beach and strong currents may have given litter no chance to accumulate.

At Faksevågen the high number of very small plastic items, often entangled in floating weed washed ashore may be a result of the beach acting as a sink for marine litter. Faksevågen is located near the final part of the North Atlantic (NA) current. It is possible that floating debris (both natural and anthropogenic) collects on this beach after being broken into smaller pieces during transport on the NA current.

Plastic was the dominant form of anthropogenic debris found. This included small plastic or polystyrene pieces, nets and pieces of net, industrial packaging, caps and lids, and strapping bands (Figure 11). Most of these items appear to be related primarily to the fisheries industry, an observation that has been reported before (Falk-Andersson et al., 2019). Identifying the source of litter is essential for developing appropriate and effective policies to reduce and prevent the amount of litter currently entering the environment.

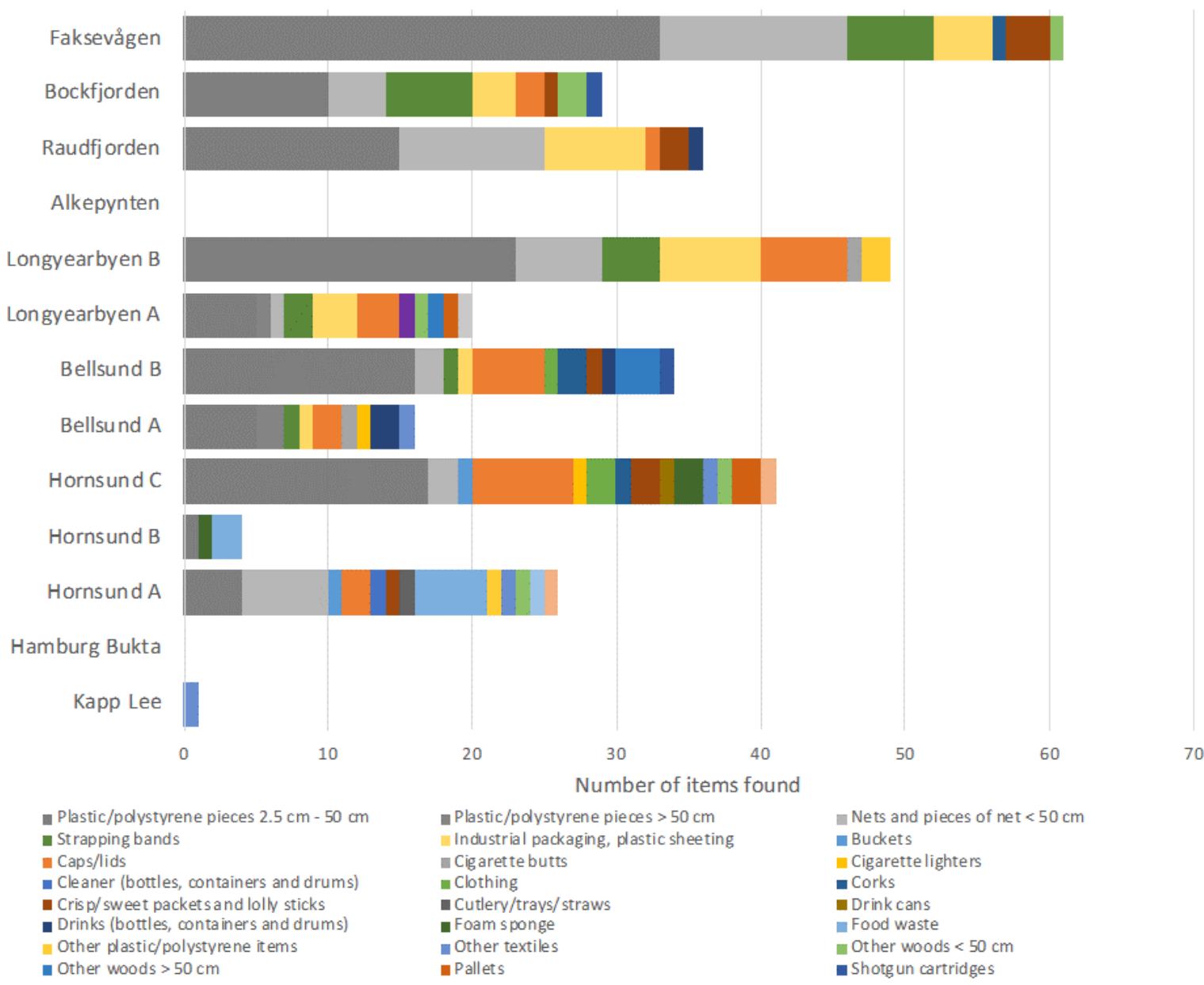

Figure 10 Number and types of anthropogenic debris found on different beaches during a standardised 10 minute transect search by three people, as surveyed on the beaches of Svalbard in 2018. Debris categories were taken from the OSPAR beach litter classification method (OSPAR, 2010). 


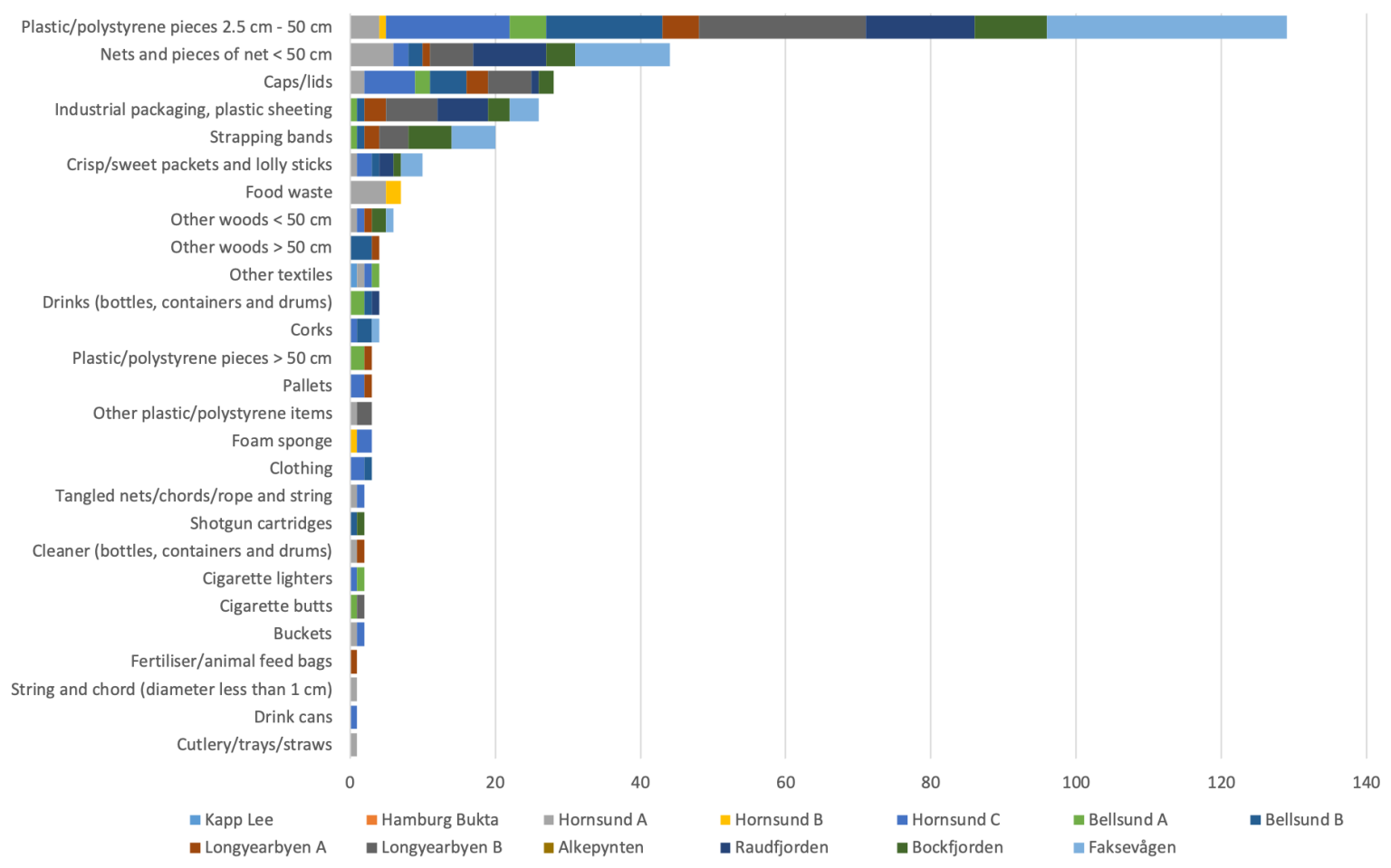

Figure 11 Total number and type of anthropogenic debris found during a standardised 10 minute transect search by three people, and the beaches on which they were found, as surveyed on the beaches of Svalbard in 2018. Debris categories were taken from the OSPAR beach litter classification method (OSPAR, 2010).

\subsubsection{Metabarcoding}

A total of 164 species were detected on the debris using the metabarcoding technique. Debris found at Hamberg Bukta and Rijpfjorden was classified as natural debris, while debris at all other locations was classified as artificial debris.

In general, the number of species found per sample was highest in Kapp Lee and lowest in Hamberg Bukta (natural debris), Hornsund A, Bellsund A, Longyearbyen A and Rijpfjorden (natural debris) (Figure 12). The items found on Kapp Lee was a piece of neoprene, located at a spot where beach sediment connects to terrestrial soil. The number of species varied between locations with the highest number detected in Hornsund $\mathrm{C}$ and the fewest in Hamberg Bukta (natural debris) (Figure 13).

The top ten most abundant species consisted of five species from the phylum Ochrophyta, and one species from each of the phyla Nematoda, Cercozoa, Platyhelminthes, Arthropoda and Basidiomycota (Table 12). 


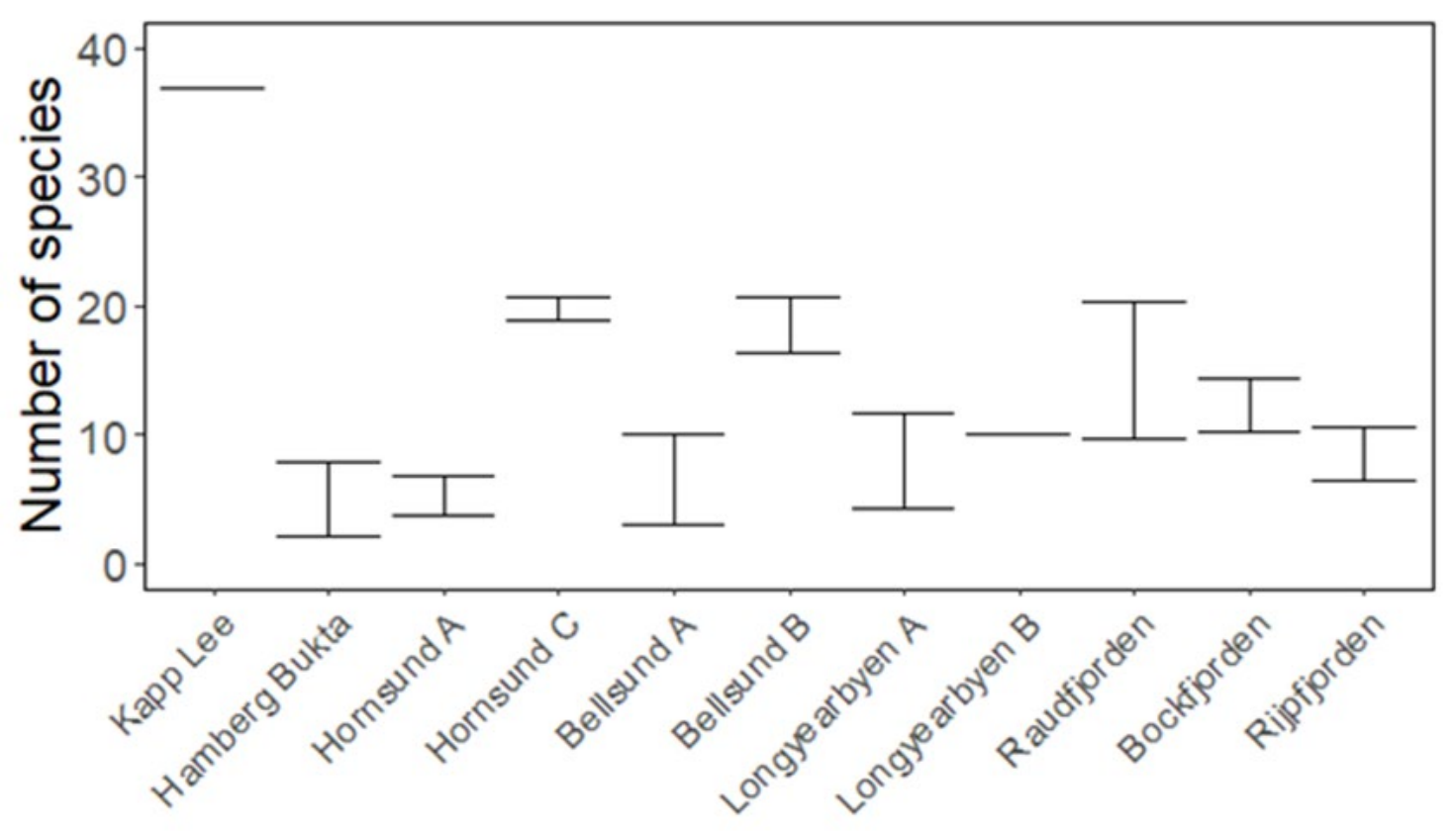

Figure 12 Number of species from different phyla and family detected using eDNA metabarcoding on selected debris items as surveyed on the beaches of Svalbard in 2018 (note that type and number of debris items varied between locations, and debris found at Hamberg Bukta and Rijpfjorden were classified as natural debris while debris at all other locations was classified as artificial debris).

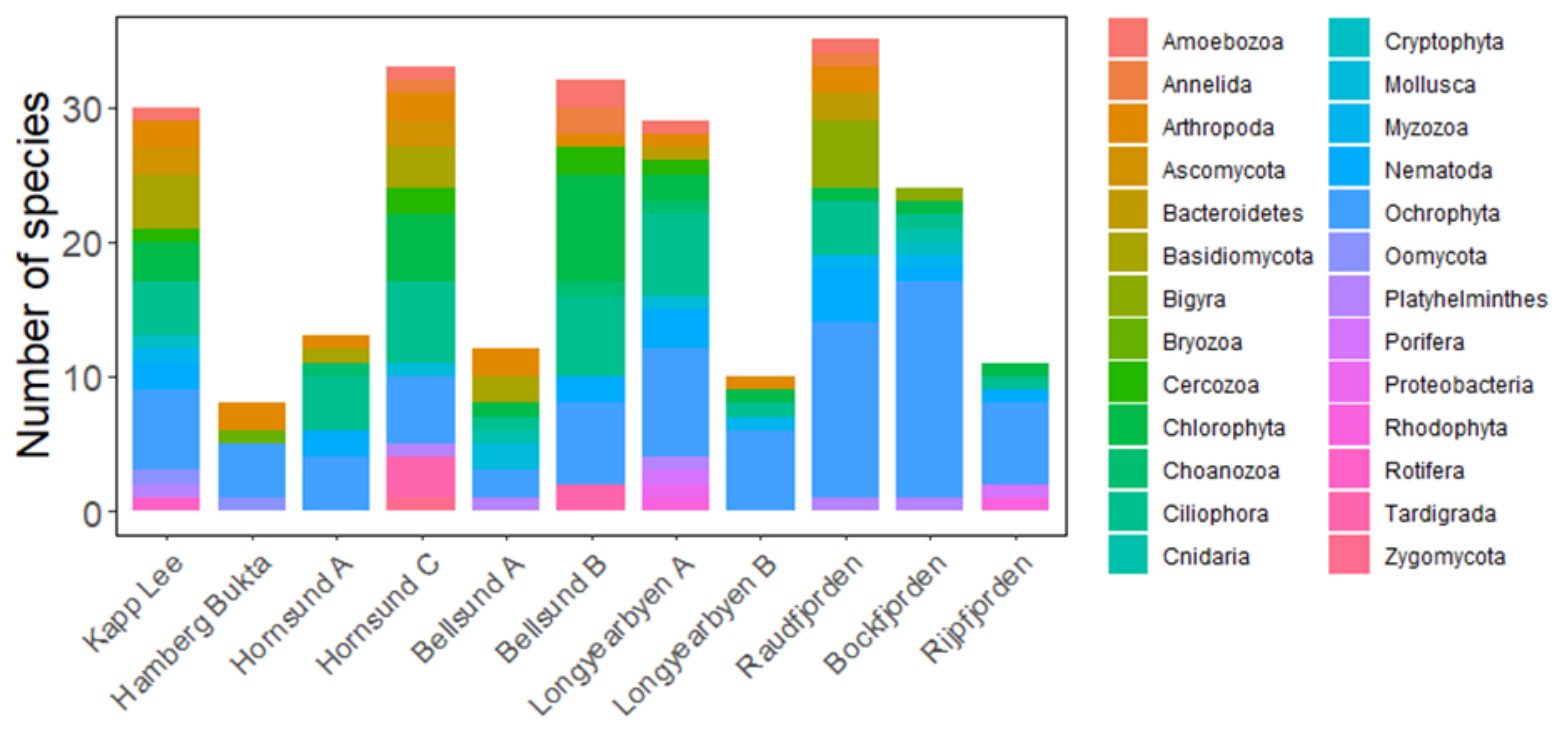

Figure 13 Number of species from different phyla and family detected using eDNA metabarcoding on selected debris items as surveyed on the beaches of Svalbard in 2018 (note that type and number of debris items varied between locations, and debris found at Hamberg Bukta and Rijpfjorden were classified as natural debris while debris at all other locations was classified as artificial debris). 


\section{Table 12}

Top ten species with positive identification in 28 collected debris samples based on eDNA metabarcoding, Svalbard in 2018.

\begin{tabular}{|l|l|c|c|}
\hline Latin name & Phylum & $\begin{array}{l}\text { \# samples } \\
\text { identified }\end{array}$ & \%samples \\
\hline Halomonhystera sp. & Nematoda & 14 & 50 \\
\hline Pylaiella littoralis & Ochrophyta & 9 & 32 \\
\hline Fucus sp. & Ochrophyta & 8 & 29 \\
\hline Saccharina sp. & Ochrophyta & 7 & 25 \\
\hline Ectocarpus sp. & Ochrophyta & 7 & 25 \\
\hline Bodomorpha sp. & Cercozoa & 7 & 25 \\
\hline Astrotorhynchus sp. & Platyhelminthes & 7 & 25 \\
\hline Semibalanus balanoides & Arthropoda & 6 & 21 \\
\hline Licmophora juergensii & Ochrophyta & 6 & 21 \\
\hline Dioszegia sp. & Basidiomycota & 6 & 21 \\
\hline
\end{tabular}

\subsection{Metabarcoding data combined}

A total of 293 species in 106 samples from sediment, hard substrate and debris were identified using the eDNA metabarcoding technique. The locations with highest number of species were Bellsund $B, A$ and Longyearbyen B (Figure 14). Per sample more species were found in soft sediment samples than in hard substrate and debris (Figure 15).

Sampling the intertidal sediment may have limited the number of species detected. Some DNA in the samples had undergone degradation, probably as a result of regular exposure to air and UV light, and could therefore not be analysed in as much detail as if the samples had been from sublittoral sediment. In an earlier study in sublittoral sediment samples of Kongsfjorden, Svalbard, 299 species were identified from 30 samples using similar DNA metabarcoding techniques (Van den Heuvel-Greve et al., 2021), whereas in our study 157 species were identified in 52 intertidal sediment samples.

Future sampling should therefore focus on sublittoral sediment samples to be able to obtain sediment samples with less degraded DNA.

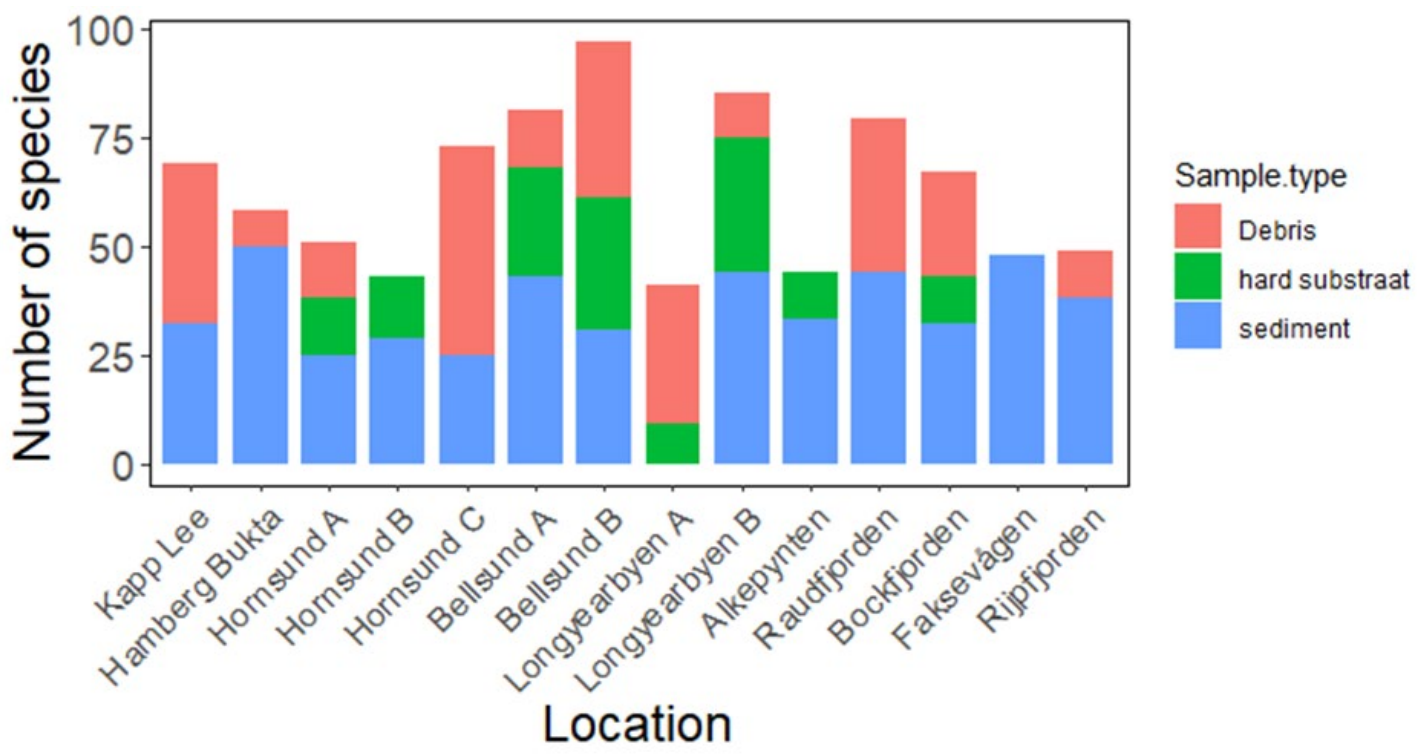

Figure 14 Number of species found on and in debris, hard substrate and sediment samples that were collected on beaches around Svalbard in 2018, per sampled location. 


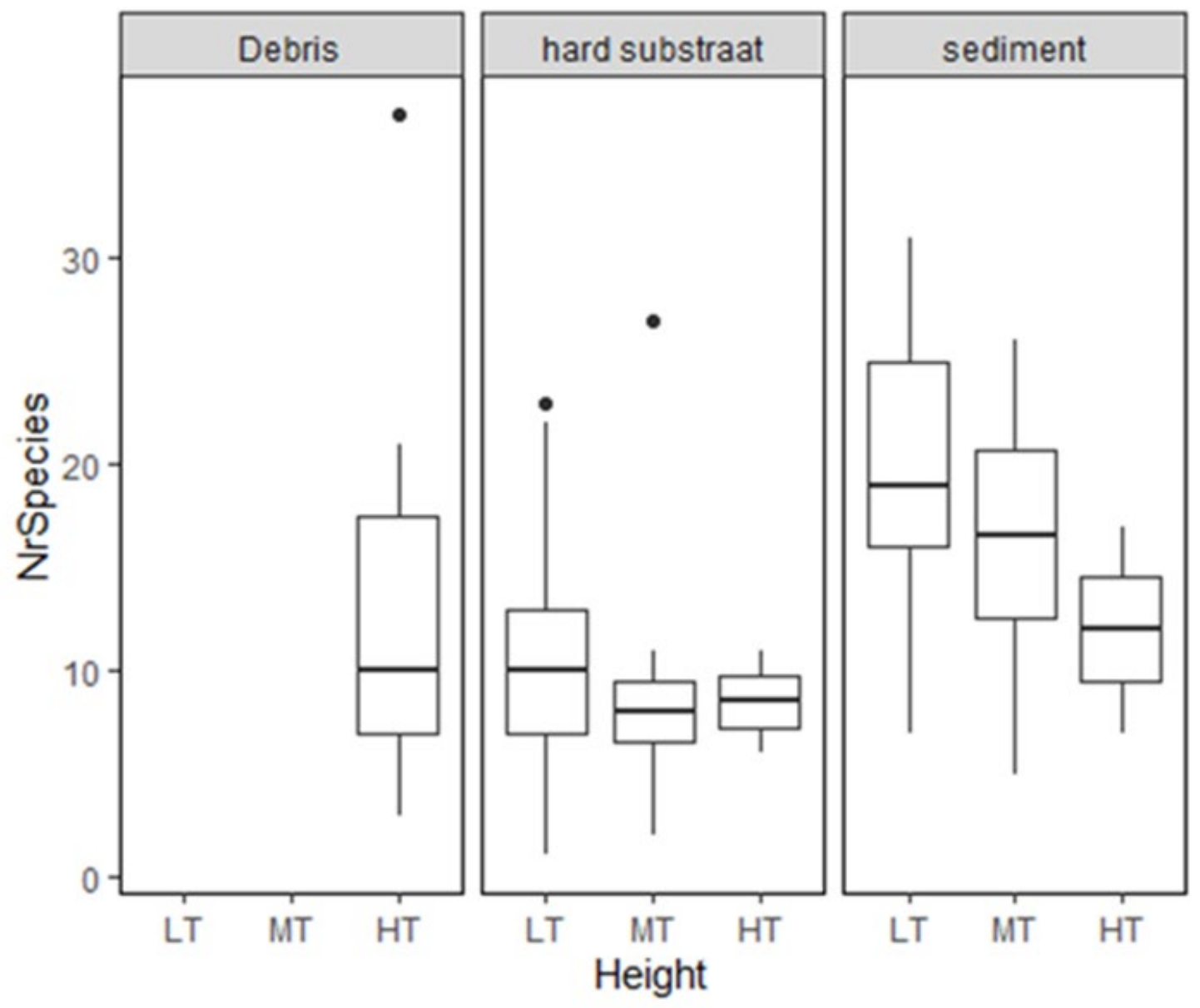

Figure 15 Number of species found on and in debris, hard substrate and sediment samples that were collected on beaches around Svalbard in 2018, divided per substrate type and height on the beach.

\subsubsection{Species composition}

When combining all metabarcoding data, regardless of sediment type or height, there was a significant difference $(p<0.05)$ in species composition between locations. Several locations were similar in species composition, except Hornsund C, Longyearbyen A, Bellsund B and Kapp Lee which seem to differ from the rest (Figure 16), but due to strong correlations in the data, multivariate analyses were not possible to test which locations differed from each other. There was no obvious factor in physical characteristics of these beaches explaining the differences in species composition compared with the other beaches. There were no characteristics common to only these four beaches and not the others (Table 5 and Table 6).

Graphs were also plotted separately for soft sediment, hard substrate and debris. As sample sizes were small, no statistical analyses could be performed. However, when analysing soft sediment $(n=52)$, species composition appeared to be particularly different in Longyearbyen $B$ and Bellsund $B$. Species composition was more similar in Hornsund $C$ and Rijpfjorden. Hard substrate $(n=26)$ showed that Longyearbyen B and Bellsund B were slightly similar, but differed from the rest. The other locations differed slightly where the species composition was most similar in Bockfjorden and Alkenpynten. The species composition found on debris $(n=28)$ was most similar between Rijpfjorden and Longyearbyen A, or Bellsund A and Hornsund A, or Bellsund B and Hornsund C. Locations at which the composition differed from the rest were Kapp Lee and Hamberg Bukta.

Species composition was significant different $(p<0.05)$ among substrate types. Species composition found on debris and hard substrate were more similar, as they were closer together on the graph compared to soft sediment (Figure 17). 


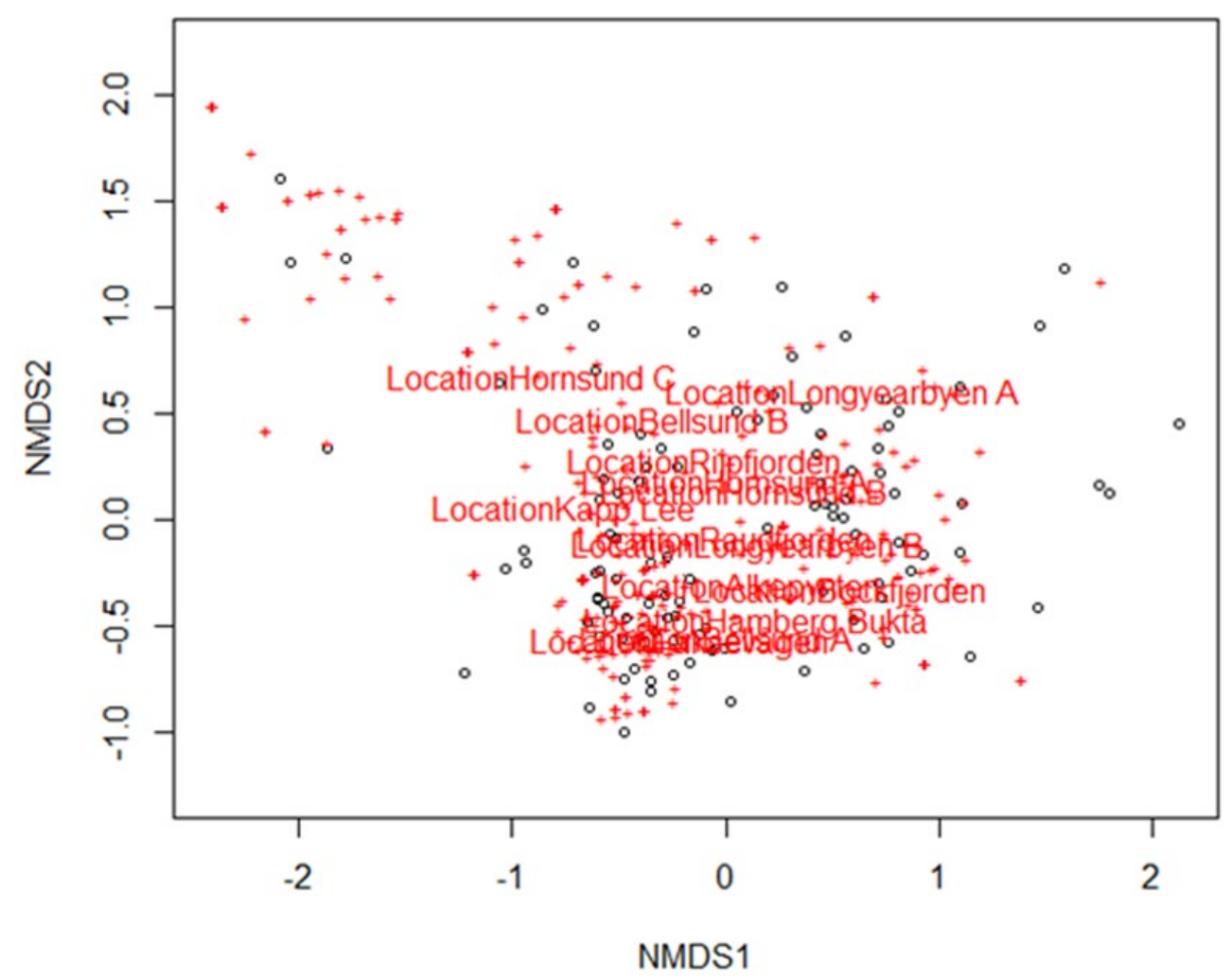

Figure 16 nMDS plot for species composition of samples (based on eDNA) that were collected on Svalbard beaches in 2018 ( $n=106)$. Locations that are on top of each other are very similar in species composition. The farther apart two locations are on the graph, the greater the difference in species composition.

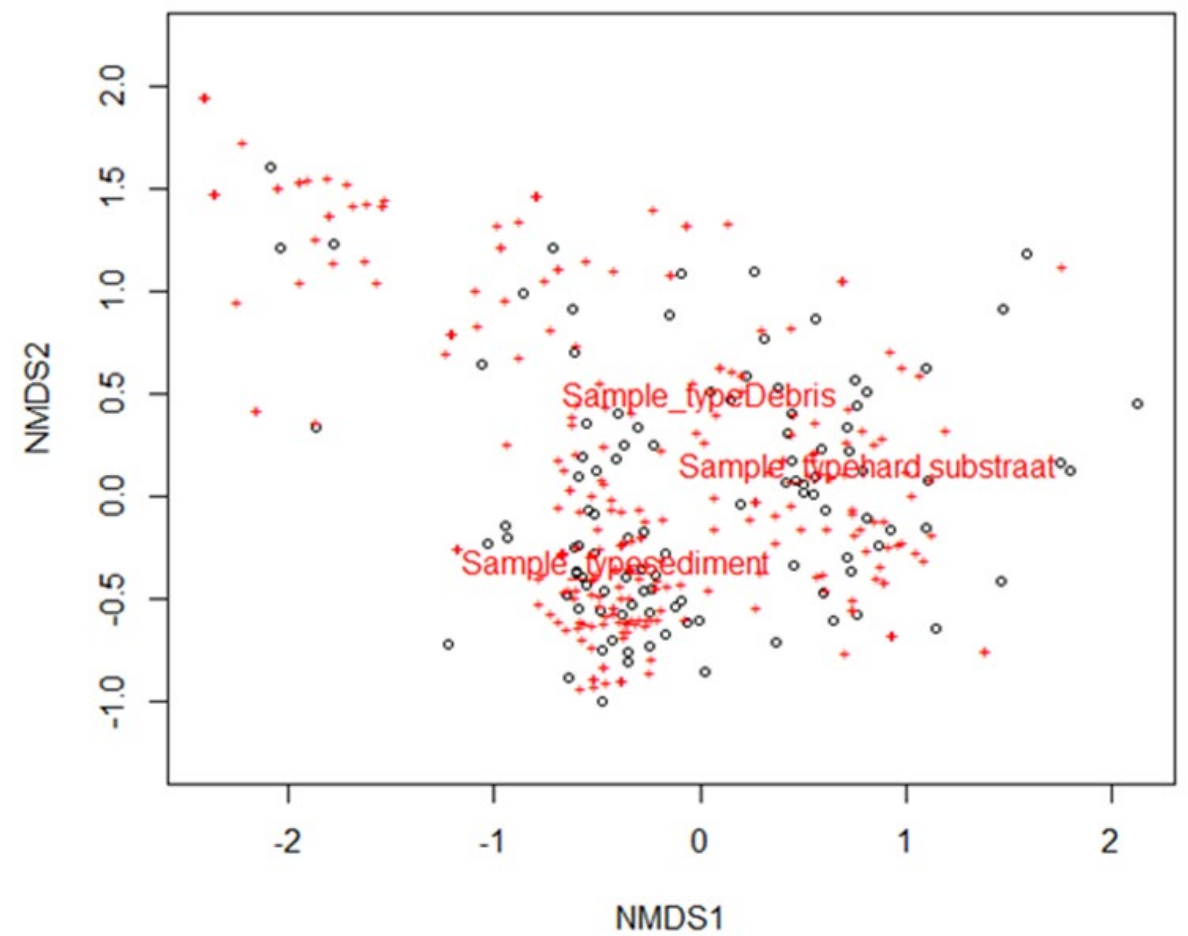

Figure 17 nMDS plot for species composition of samples (based on eDNA) that were collected on Svalbard beaches in 2018 from debris, hard substrate and soft sediment $(n=106)$. 
Species composition was significantly different $(p<0.05)$ between the heights at which the samples were collected (Figure 18). Species composition seemed more similar between LT and MT and more different at HT. These differences between HT and the other tidal heights might also be a result of the sampling scheme as fewer samples were collected on this line. In addition, debris was only found at the high-water line and not at the low or mid-water line which might also have an effect on the results.

\subsection{Non-indigenous species}

In all substrate types only Euterpina acutifrons could be linked to the list of potential NIS for Svalbard (Table 13) (Van den Heuvel-Greve at al., 2021). These were also identified in sediments in an earlier study using metabarcoding techniques (Van den Heuvel-Greve, 2021). Five others were identified to the genus level, but for four of these genera, native species were also reported, so no further identification could be made based on these results. For instance, Calanus sp. have three native species in the region, Fucus distichus is the native Fucus species in the Arctic, and Pseudocalanus minutus, Pseudocalanus acuspes, and Pseudocalanus major are very common to rarely found in Svalbard fjords (Table 13). Mytilus sp. has been reintroduced to Svalbard and is therefore considered an NIS (Van den Heuvel-Greve et al., 2021).

Euterpina acutifrons was detected in a hard substrate sample in Hornsund A. More frequently Euterpina sp. was observed in hard substrate samples. The Mytilus sp. was found on two debris samples in Bellsund A and Longyearbyen A.

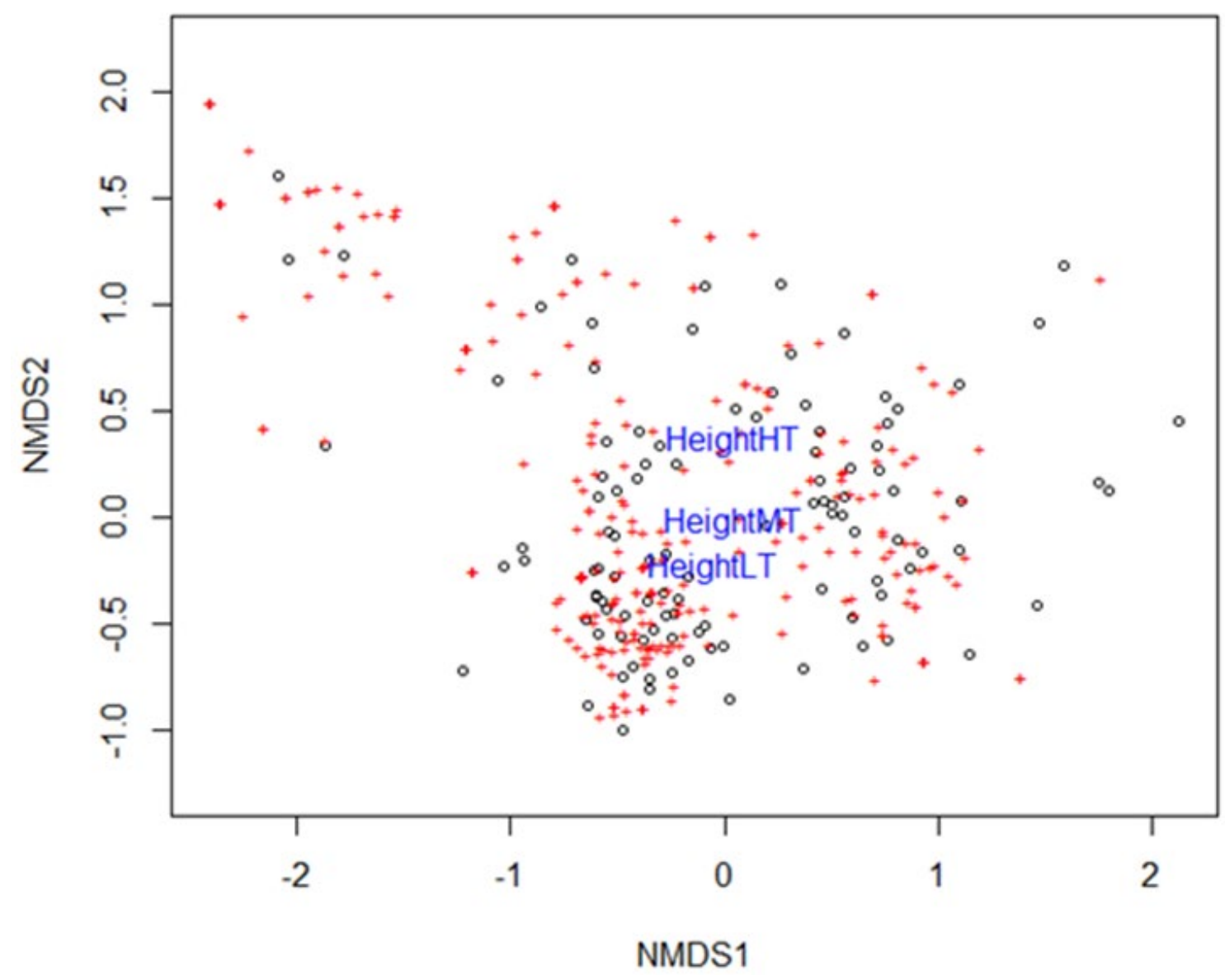

Figure 18 nMDS plot for species composition of samples (based on eDNA) that were collected on Svalbard beaches in 2018 at different heights on the beach: HT (high water line), MT (mid-water line) and LT (low water line) ( $n=106)$. 


\section{Table 13}

Detection of potential NIS for Svalbard in the samples collected at a variety of beaches around Svalbard in 2018. The column of potential NIS is based on the list of potential marine NIS for Svalbard (Van den Heuvel-Greve et al., 2021).

\begin{tabular}{|c|c|c|c|}
\hline Phylum & Potential NIS & $\begin{array}{l}\text { Identified in the } \\
\text { samples }\end{array}$ & Native species \\
\hline Arthropoda & Calanus helgolandicus & Calanus sp. & $\begin{array}{l}\text { C. glacialis, C. hyperboreus, } \\
\text { C. finmarchicus }\end{array}$ \\
\hline Arthropoda & Euterpina acutifrons & $\begin{array}{l}\text { Euterpina acutifrons, } \\
\text { Euterpina sp. }\end{array}$ & - \\
\hline Arthropoda & Pseudocalanus & Pseudocalanus sp. & Pseudocalanus spp. \\
\hline Ochrophyta & Fucus cottonii & Fucus sp. & Fucus distichus \\
\hline Ochrophyta & Fucus serratus & Fucus sp. & Fucus distichus \\
\hline Mollusca & Mytilus galloprovincialis & Mytilus sp. & - \\
\hline
\end{tabular}

\subsection{General discussion}

\subsubsection{Habitat classification of Svalbard beaches}

Habitat classification of beaches around Svalbard was conducted according to a newly developed methodology:

- The beaches around Svalbard vary greatly in physical characteristics, particularly in sediment grain size, orientation, slope and exposure. Based on the observed variety in beach type, no characteristics could yet be reliably associated with a risk of introducing NIS.

- The intensity and type of human visitation also varied greatly between beaches. All beaches had human activities in the neighbourhood (tourism, research, fishing), except Hamberg Bukta. As the glacier at Hamberg Bukta is declining and is expected to open up a passage to the Hornsund on the west in the future, this area will likely become a thoroughfare for shipping.

- The beach descriptions presented here emphasise the wide range of beach types and have formed a good basis for further beach description. More information on the beaches around Svalbard is necessary to produce a useful standardised classification.

- $\quad$ Further research should focus on describing a higher number of beaches so that commonalities can be further identified and associated with risk levels for introducing NIS. The need for Svalbard-specific standard coastscapes was also recommended by a recent workshop on Sustainable Svalbard Coasts (Soreide et al., 2020). The frequency, and type of human traffic should be taken into account along with the physical characteristics of the beach to identify potential hotspots and focus areas for monitoring and mitigation efforts.

\subsubsection{Aliens on the beach}

- The only potential 'aliens' on the beach were Euterpina acutifrons (on hard substrate), Mytilus sp. (on two debris items) as well as possibly a few other species that were identified to a genus level. These were also identified in sediments in an earlier study using metabarcoding techniques (Van den Heuvel-Greve, 2021). Most of the species detected during the survey using metabarcoding were Arctic species.

- Monitoring efforts for NIS should preferably focus on hotspots and points of entry such as harbours and the entrance of major fjord systems on the North Atlantic side of Svalbard (Hornsund, Van Mijenfjorden, Isfjorden and Kongsfjorden) (Van den Heuvel-Greve et al., 2021). 
- The identified species on various types of debris via metabarcoding techniques suggest that floating debris can play a role as a vector of dispersal and introduction of NIS and repatriates (such as blue mussels) (Węsławski \& Kotwicki, 2018; Van den Heuvel-Greve et al., 2021). The exposition - or fetch - is a critical parameters for the debris on the beach. Generally especially in autumn - the wrack (macroalgae) can be deposited anywhere, yet there are some sites where the debris accumulates (such as the driftwood and plastic). These are strongly influenced by currents, fetch, or exposure and a combination of these. Although it does not tell that those biological objects are living nearby (for instance since many years Ascophyllum bladder wrack is found on Svalbard, though it has not been seen alive and growing on the coast), is provides information on what arrives on the coast and has the potential to settle. It is therefore also recommended to monitor beaches where marine litter is washed upon the shore in high numbers, such as Prins Karlforland (Węsławski \& Kotwicki, 2018; Van den Heuvel-Greve et al., 2021).

\subsubsection{Metabarcoding as monitoring tool}

- $\quad$ eDNA metabarcoding is a suitable tool for monitoring the presence of NIS in the Arctic provided the DNA barcode database covers the relevant barcodes for potential NIS. The use of eDNA metabarcoding provides an opportunity to sample many locations and produce a large dataset of organism identification en masse without the labour and expertise necessary for morphological identification. The metabarcoding method also generally allowed identification of organisms to a lower taxonomic level than morphological identification, which is important as NIS may share the same genus as native species. However morphological identification of species produced a different list of organisms present in the same area. The use of both morphological identification and DNA metabarcoding together is therefore recommended to produce complimentary data and a more thorough insight into the biodiversity of the beach than using one method exclusively.

- The results of this pilot study provide a baseline of the species present in the area at this point in time, and can be used for comparison with future monitoring data sets. This will be especially useful as the changes in the Arctic environment continue and shipping and other human activities increase.

- The samples of intertidal beach sediment show some degradation of DNA, likely due to the exposure to air and UV light. This made metabarcoding more difficult and less accurate. Further research should therefor ideally focus on subtidal sediment samples to reduce this DNA degradation.

\subsubsection{Tourism and NIS}

- Given the nature of the tourist industry on Svalbard, tourists are likely to land at beaches almost all-around Svalbard (Figure 19). The only area sampled during this study where tourists were unlikely to land was Hamberg Bukta. This ubiquity of destinations around Svalbard suggests that an introduction of NIS is likely to be spread around the archipelago through boating activities, unless quickly mitigated.

- No recent data on tourist visits were available for this study. More specific information on tourist numbers, activities and visits provided by the tourist industry would enable a further association between the influence of tourism and the potential risk or causes of NIS introduction.

- With the ubiquity of tourist landings around Svalbard in mind, tourists should be well informed about the risk on introducing NIS. Anecdotal evidence during our expedition suggested that even though the tourists were instructed to clean their boots prior to and after landing, they were not effectively informed why. Visitors taking part in ecotourism should be, and are by nature, open to being educated on the reasons for preventing the introduction of terrestrial and marine NIS.

- Additionally, tourist companies should be well educated about the risk of introducing marine NIS. These companies should be encouraged to be vigilant about their activities and locations 
of visits, and conduct regular thorough vessel cleaning, including zodiacs (fouling of hull and ballast water). More detailed and publicly available recordkeeping of tourist landings would help policy makers identify locations within which to focus monitoring and mitigation efforts.

- Similar risks for the introduction and spread of marine NIS apply to other, non-tourism boating companies, such as shipping companies and fishing fleets. Due to these risks, the personnel in these companies should also be educated on the risks and mitigation potential for marine NIS.

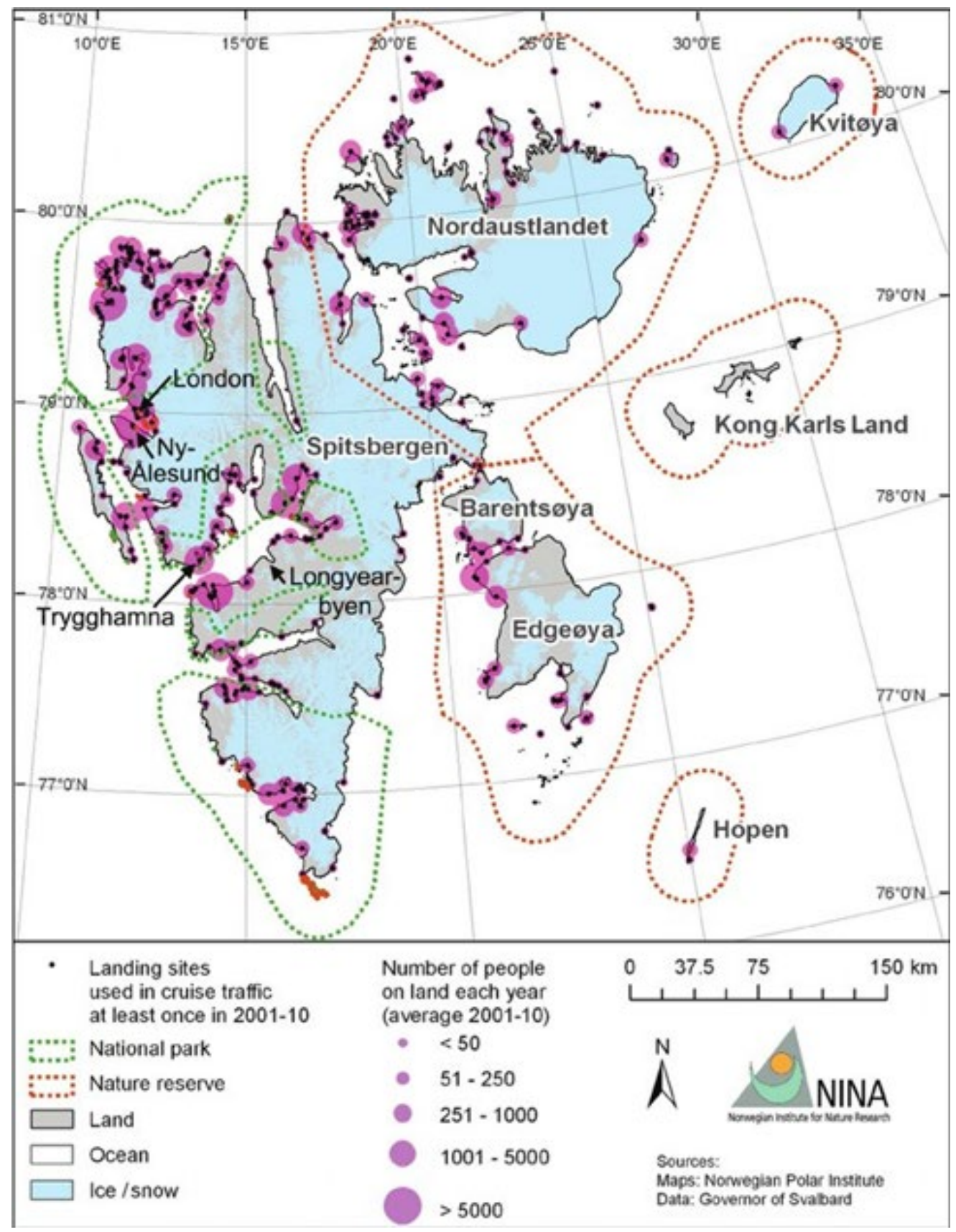

Figure 19 Areas around Svalbard visited by tourists between 2001-2010 (Hagen et al., 2012). 


\section{Conclusion and recommendation}

- Svalbard contains a wide range of beach types (sediment grain size, orientation, slope and exposure). Information on additional beaches around Svalbard is needed to produce a more holistic standardised classification.

- The Svalbard archipelago is perhaps not as remote as it is often thought to be. Regular human activities occur all around the archipelago, mainly in the form of tourism, research, fishery or shipping. The risk of NIS being introduced via human transport is therefore a concern for the local environment. As climate change causes sea-ice to melt and previously blocked passages to open up, human visitation will only increase, and with it, the likelihood of introducing NIS.

- The differences in organisms identified between eDNA metabarcoding and morphological identification indicate that both techniques are highly suitable to be used in combination, hereby producing complimentary data.

- Improving awareness of the vectors, risks and potential impacts of NIS in the tourism, fishery, shipping and research industries is necessary to most effectively implement mitigation measures.

- Regular and thorough monitoring is needed to be able to mitigate the introduction and spread of NIS. Monitoring should focus on:

- Subtidal sampling

- Hotspots and points of entry

- Complementary tools: metabarcoding providing an alternative perspective in monitoring to morphological identification. Using both tools together produce more thorough information than using either tool exclusively.

Regular monitoring will reduce the risk of missing an introduction and an NIS becoming established before mitigation efforts become redundant. 


\section{Acknowledgements}

Relevant RIS project registrations for field work on Svalbard for this proposal are:

- $\quad$ RiS project 11004 - Svalbard beach life; assessing beach habitats around Svalbard (Wageningen Marine Research)

- $\quad$ RiS number 3423 - Svalbard Intertidal Project (SIP ) (IOPAN) (the Svalbard Intertidal Project of IOPAN with a support from Miljovernfond Sysselmannen http://water.iopan.gda.pl/projects/SIP/index.html)

This project was funded by:

- $\quad$ Svalbard Environmental Protection Fund (18/35 - Aliens on the beach)

- Knowledge Base Programme of Wageningen University and Research (431.830.0049)

- Norwegian Environmental Department, University Centre in Svalbard, Akvaplan-niva

- $\quad$ ADAMANT project - DAINA - Polish-Lithuanian Funding Initiative, NCN 2017/27/L/NZ8/03331 Sampling permission was granted by the Governor of Svalbard (11.07.201, 18/00834-2). 


\section{Quality Assurance}

Wageningen Marine Research utilises an ISO 9001:2015 certified quality management system. This certificate is valid until 15 December 2021. The organisation has been certified since 27 February 2001. The certification was issued by DNV GL. 


\section{References}

- $\quad$ Blackburn, T.M., F. Essl, T. Evans, P.E. Hulme, J.M. Jeschke, I. Kühn, S. Kumschick et al (2014). A unified classification of alien species based on the magnitude of their environmental impacts. PLoS biology 12, no. 5: e1001850.

- Evenset, A., P.E. Renaud, G.N. Christensen. 2017. Stasjonsnett for basisovervåking på Svalbard. Akvaplan-niva rapport nr. $9127-1.26 \mathrm{~s}$.

- Falk-Andersson, J., W.J. Strietman, R.B. Larsen, G.W. Gabrielsen, E. Leemans, A. Schadeberg, E.R. Johannessen (2019). Svalbard Beach Litter Deep Dive. SALT report 1033, 57 pp.

- Hagen, D., Vistad, O., Eide, N.E., Flyen, A. and Fangel, K. (2012). Managing visitor sites in Svalbard: from a precautionary approach towards knowledge-based management. Polar Research 31(1): p.18432.

- Hopkins, C.C.E (2001). Actual and potential effects of introduced marine organisms in Norwegian waters, including Svalbard. Research report 2001-1. Directorate for Nature Management.

- Molnar, J., Gamboa, R., Revenga, C., Spalding, M. (2008). Assessing the Global Threat of Invasive Species to Marine Biodiversity. Frontiers in Ecology and the Environment, 6(9), 485-492.

- OSPAR Commission (2010). Guideline for Monitoring Marine Litter on the Beaches in the OSPAR Maritime Area.

- Renaud, P.E., Sejr, M.K., Bluhm, B.A., Sirenko, B., Ellingsen, I.H. (2015). The future of Arctic benthos: Expansion, invasion, and biodiversity. Progress in Oceanography 139: 244-257.

- Renaud, P.E., M. Forwick, H. Andrade, E.-C. Refit (2016). A metadata atlas for Svalbard benthos: scoping for habitat mapping studies. Akvaplan-niva Report 8057-02; Troms $\emptyset$ Norway.

- Slijkerman, D.M.E., S.T. Glorius, A. Gittenberger, B.E. van der Weide, O.G. Bos, M. Rensing, G.A. de Groot (2017). Monitoring Groningen Sea Ports, Non-indigenous species and risks from ballast water in Eemshaven and Delfzijl; Wageningen, Wageningen Marine Research report C045/17 A. 81 pp.

- Søreide, J.E. et al. (2020). Environmental status of Svalbard coastal waters; coastscapes and coastal ecosystem components (SvalCoast).

- Thomassen, J., Dahle, S., Hagen, D., Hendrichsen, D., Husa, V., Miller, A., Moe, B., Ravolainen, V., Renaud, P. E. \& Westergaard, K.B. (2017). Fremmede arter i Arktis - med fokus på Svalbard og Jan Mayen. NINA Rapport 1413, 48 pp.

- Van den Brink, A., M. Van den Heuvel-Greve, O. Bos, E. Paree, J. Wijsman, T. Ysebaert (in prep). Hospitable habitats: a case study comparison of two tidal bays.

- Van Den Heuvel-Greve, M.J., Szczybelski, A.S., van Den Brink, N.W., Kotterman, M.J., Kwadijk, C.J., Evenset, A., Murk, A.J. (2016). Low organotin contamination of harbour sediment in Svalbard. Polar Biology 39(10): 1699-1709.

- Van den Heuvel-Greve, M., A. Van den Brink, A. Blanco, S. Glorius, J. Bovenschen, A. de Groot, I. Laros, P.E. Renaud, R. Pettersen, J.M. Węsławski, P. Kuklinski, A.J. Murk (2021). Early detection of marine non-indigenous species on Svalbard by DNA metabarcoding of sediment. Polar Biology, 1-13, https://doi.org/10.1007/s00300-021-02822-7.

- Ware, C., Berge, J., Sundet, J.H., Kirkpatrick, J.B., Coutts, A.D.M., Jelmert, A., Olsen, S.M., Floerl, O., Wisz, M.S. and Alsos, I.G. (2014). Climate change, non-indigenous species and shipping: assessing the risk of species introduction to a high-Arctic archipelago. Diversity Distribution 20: 10-19.

- Ware, C., Berge, J., Jelmert, A., Olsen, S.M., Pellissier, L., Wisz, M., Kriticos, D., Semenov, G., Kwaśniewski, S., Alsos, I. G. (2016). Biological introduction risks from shipping in a warming Arctic. Journal of Applied Ecology 53: 340-349.

- Weslawski, J.M., Wiktor, J., Zajaczkowski, M., Swerpel, S. (1993). Intertidal zone of Svalbard: 1. Macroorganism distribution and biomass. Polar Biology, 13(2), 73-79.

- Węsławski J.M., Wiktor J., Zajączkowski M.,., Futsaeter G., Moe K.A. (1997). Vulnerability Assessment of Svalbard Intertidal Zone for Oil Spills. Estuarine, Coastal and Shelf Science 44 (Supplement A): 33-41. 
- Weslawski, J. M., Szymelfenig M. (1999). Community composition of tidal flats on Spitsbergen: consequence of disturbance? NATO SCIENCE SERIES 2 ENVIRONMENTAL SECURITY, 59, 185-194.

- Węsławski J.M., Wiktor J.jr., Kotwicki L. (2010). Increase in biodiversity in the Arctic rocky littoral, Sørkappland, Svalbard after 20 years of climate warming. Marine Biodiversity 40: 123- 130.

- Węsławski, J. M., \& Kotwicki, L. (2018). Macro-plastic litter, a new vector for boreal species dispersal on Svalbard. Polish Polar Research: 165-174.

- Wiencke, C., Hop, H. (2016). Ecosystem Kongsfjorden: new views after more than a decade of research. Polar Biology 39: 1679-1687.

- Wiktor J., Tatarek A., Weslawski JM., Kotwicki L., Poulin M., 2016. Colonies of Gyrosigma eximium: a new phenomenon in Arctic tidal flats. Oceanologia 58(4): 336-340.

- Zaiko, A., Olenin, S., Daunys, D., Nalepa, T. (2007). Vulnerability of benthic habitats to the aquatic invasive species. Biological invasions 9(6): 703-714. 


\section{Justification}

Report C028/21

Project Number: 431.310.0090 SEPF Aliens on the Beach; 18/35 - Aliens on the beach

The scientific quality of this report has been peer reviewed by a colleague scientist and a member of the Management Team of Wageningen Marine Research

Approved: $\quad$ Ainhoa Blanco

Scientist

Signature:

Date: 23-03-2021

Approved: $\quad$ Tammo Bult

Director

Signature:

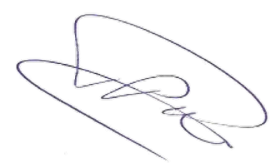

Date: 23-03-2021 


\section{Annex 1. Detailed maps of sampling locations}

Isfjorden - Alkepynten

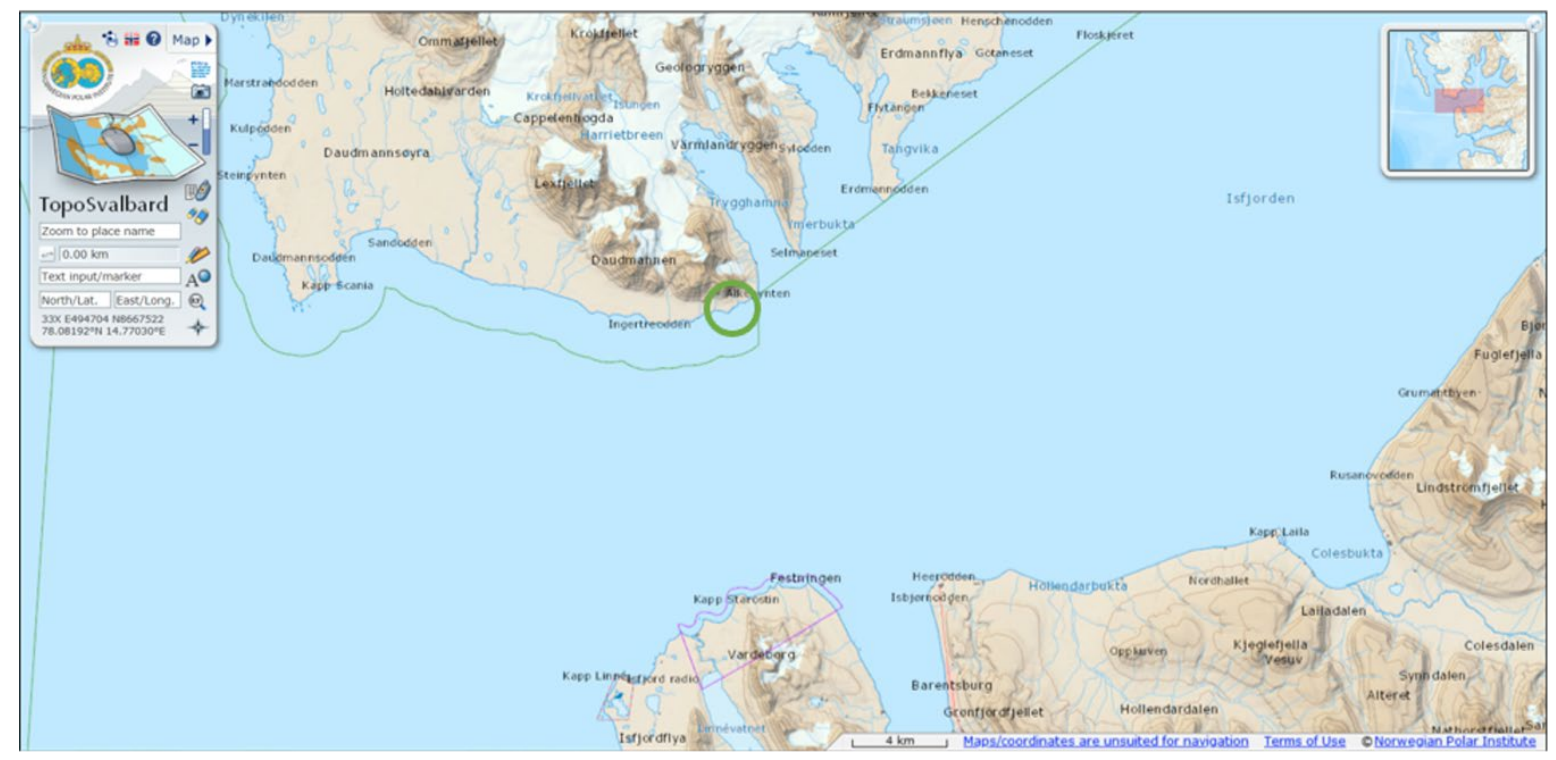

Bellsund $-\mathrm{a} / \mathrm{b} / \mathrm{c}$

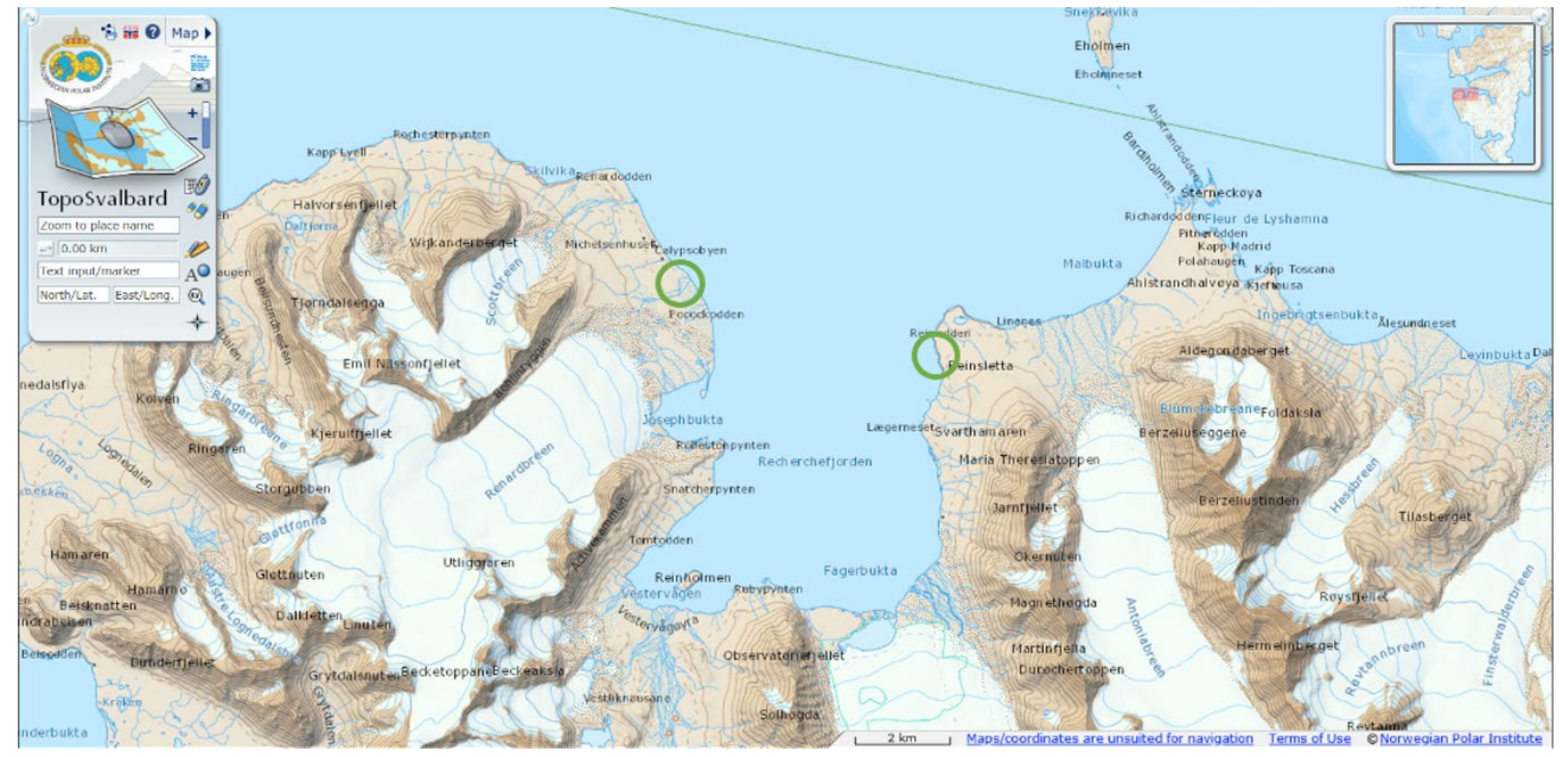




\section{Liefdefjorden - Bockfjorden}

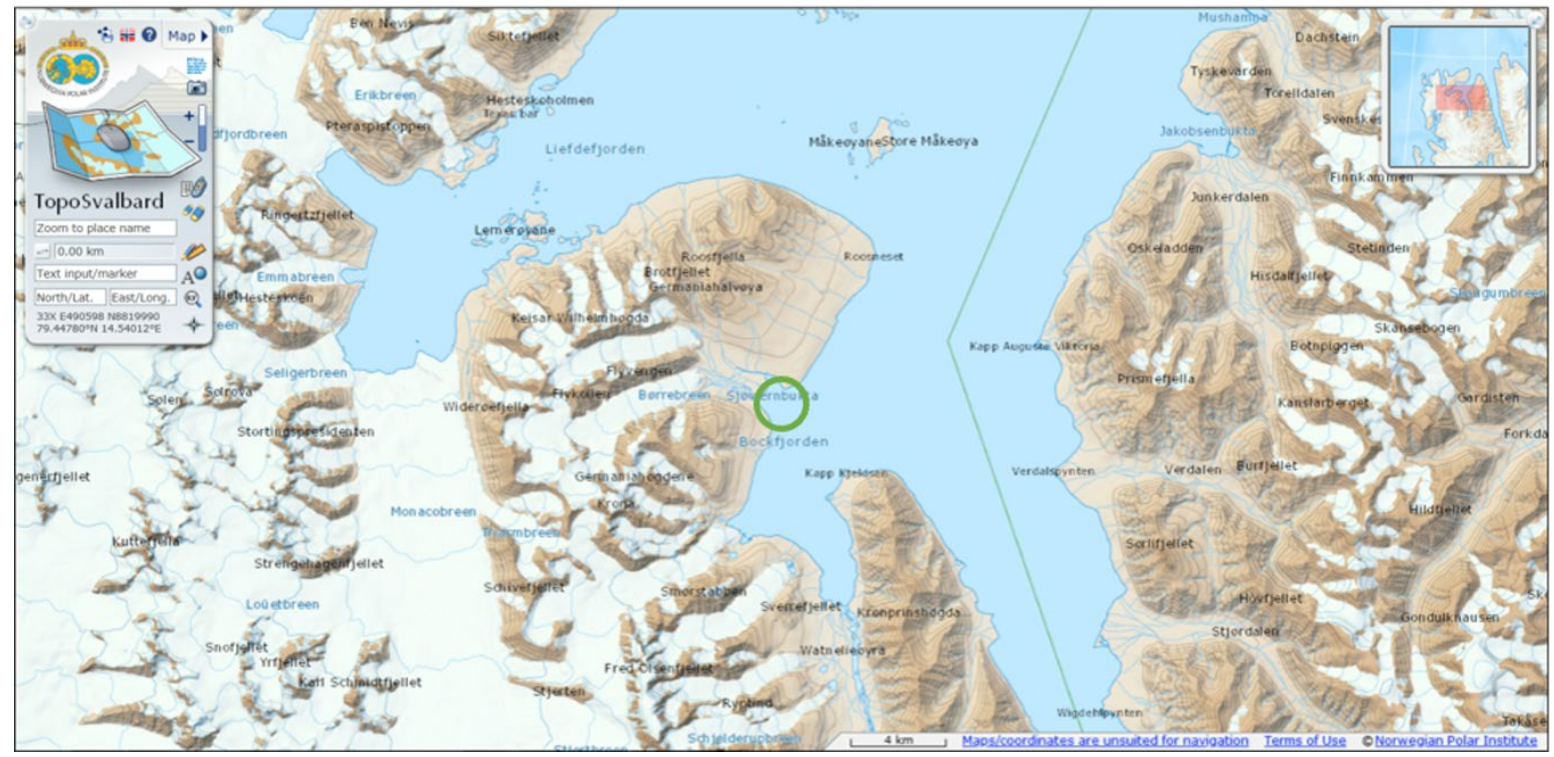

Hinlopen Strait - Faksevågen

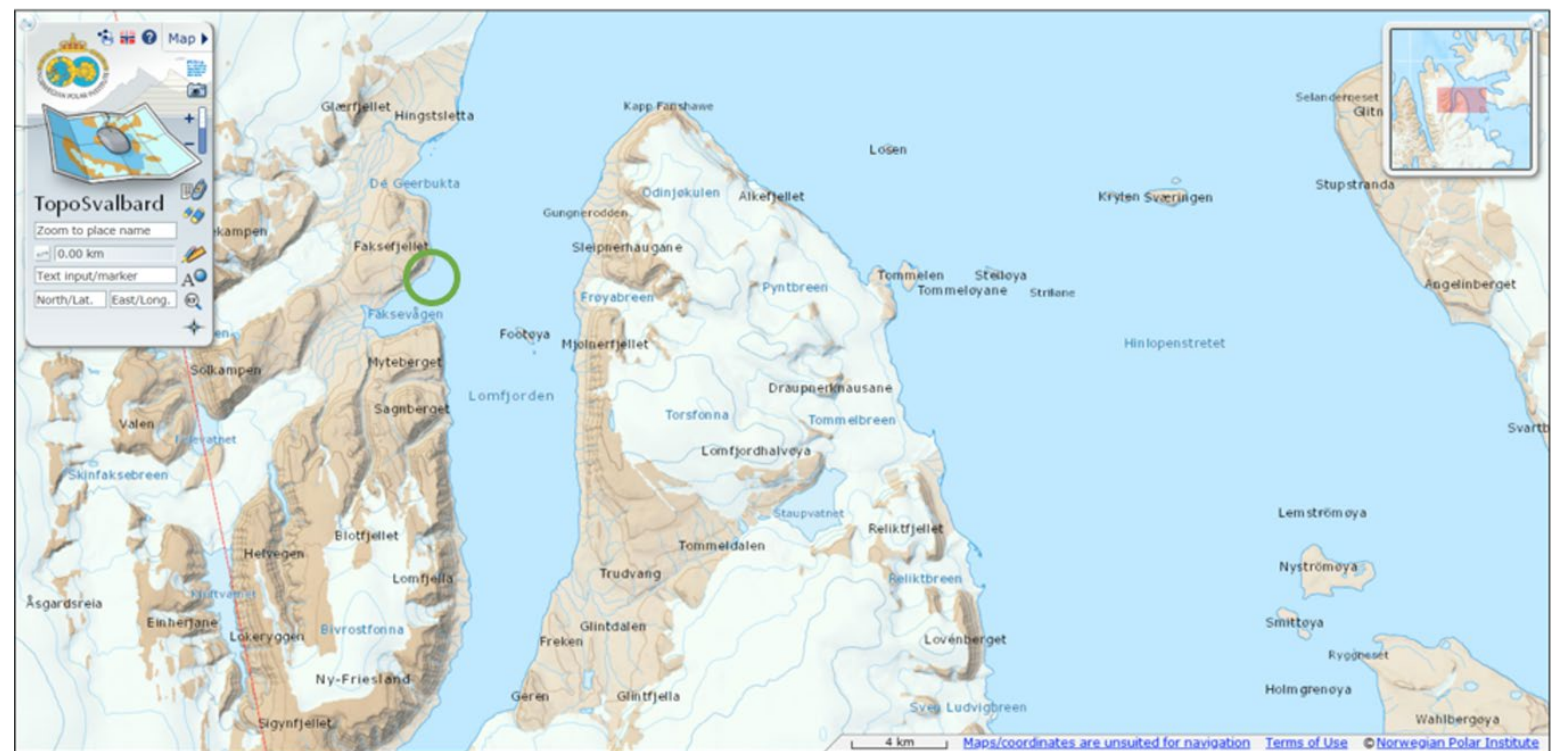

Storfjorden - Hamberg Bukta
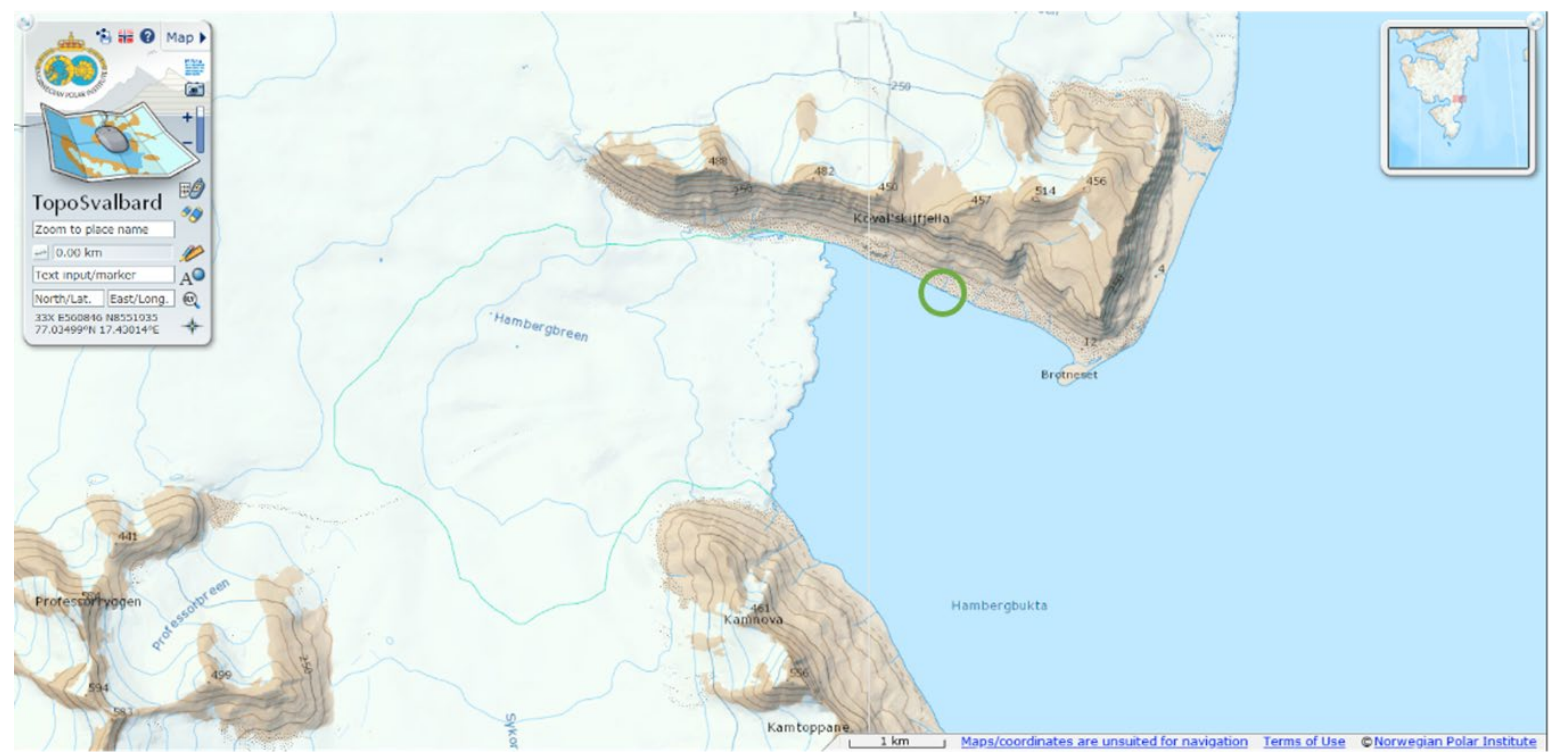


\section{Hornsund - a/b/c}

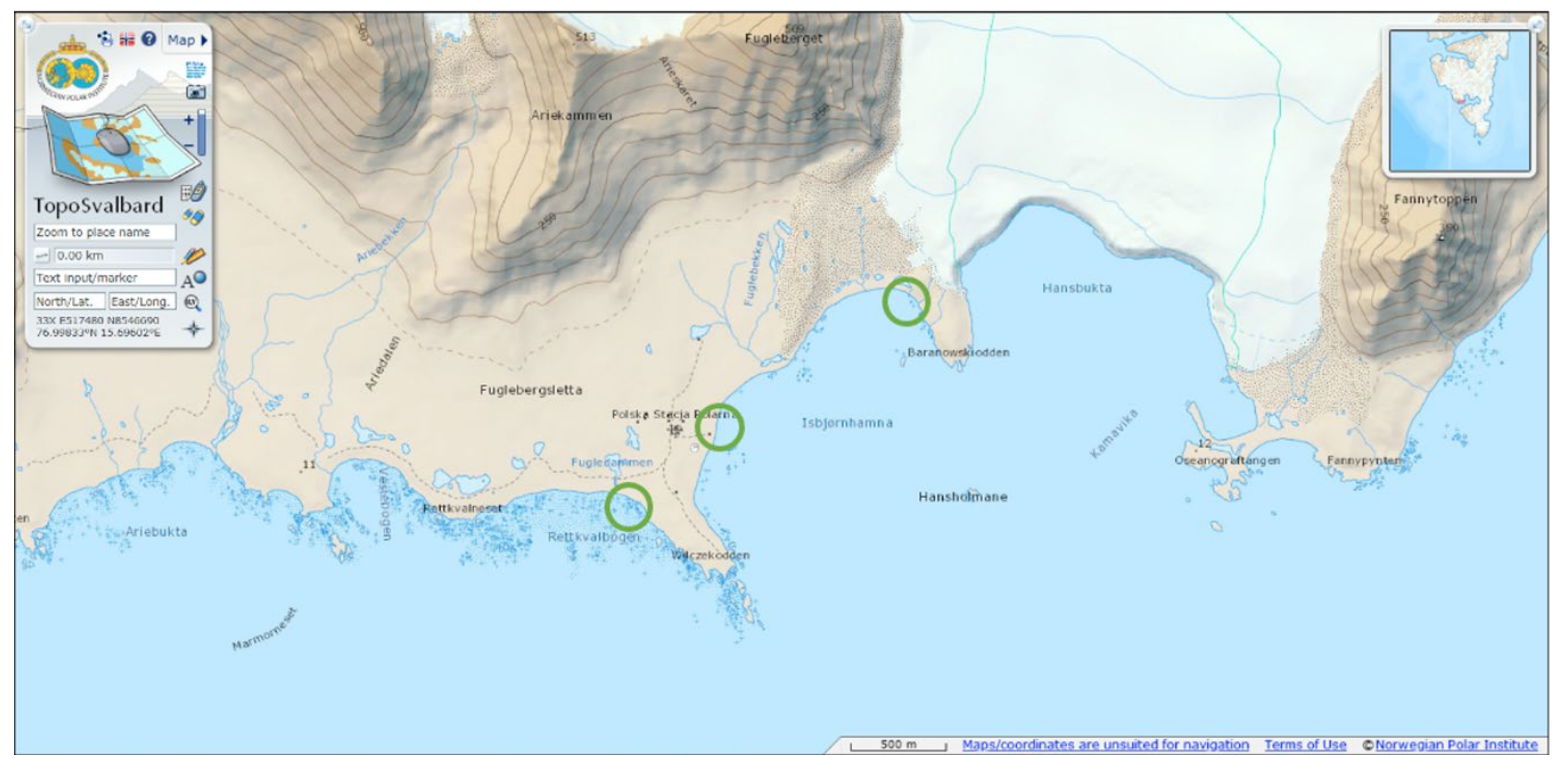

\section{Storfjorden - Kapp Lee}

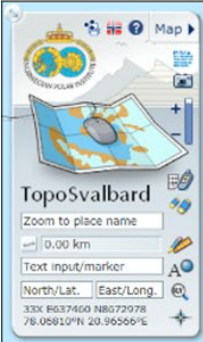

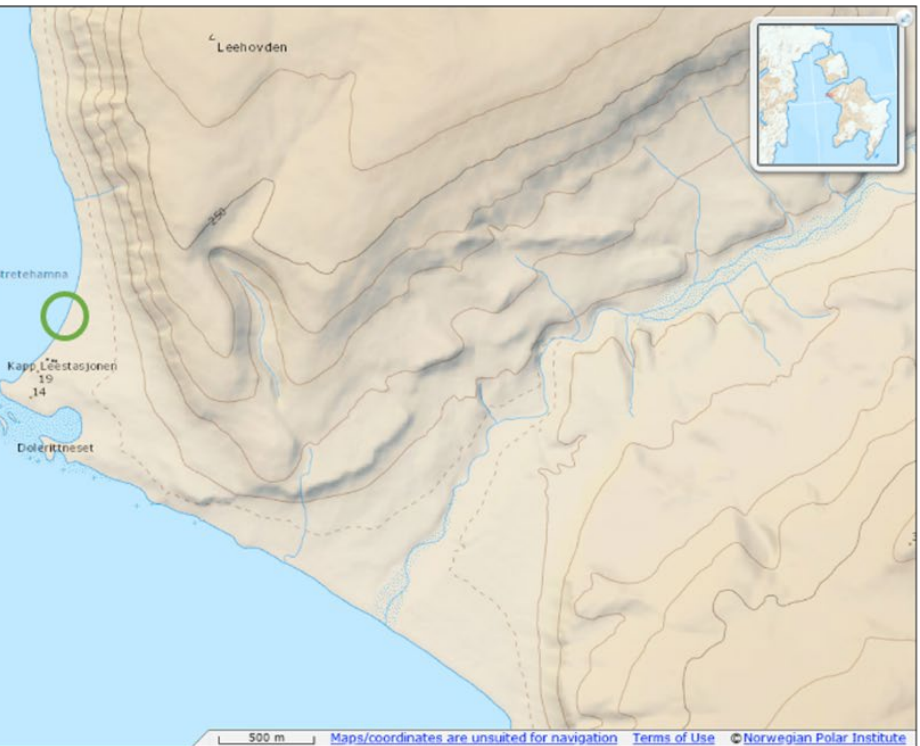

Isfjorden - Longyearbyen a/b

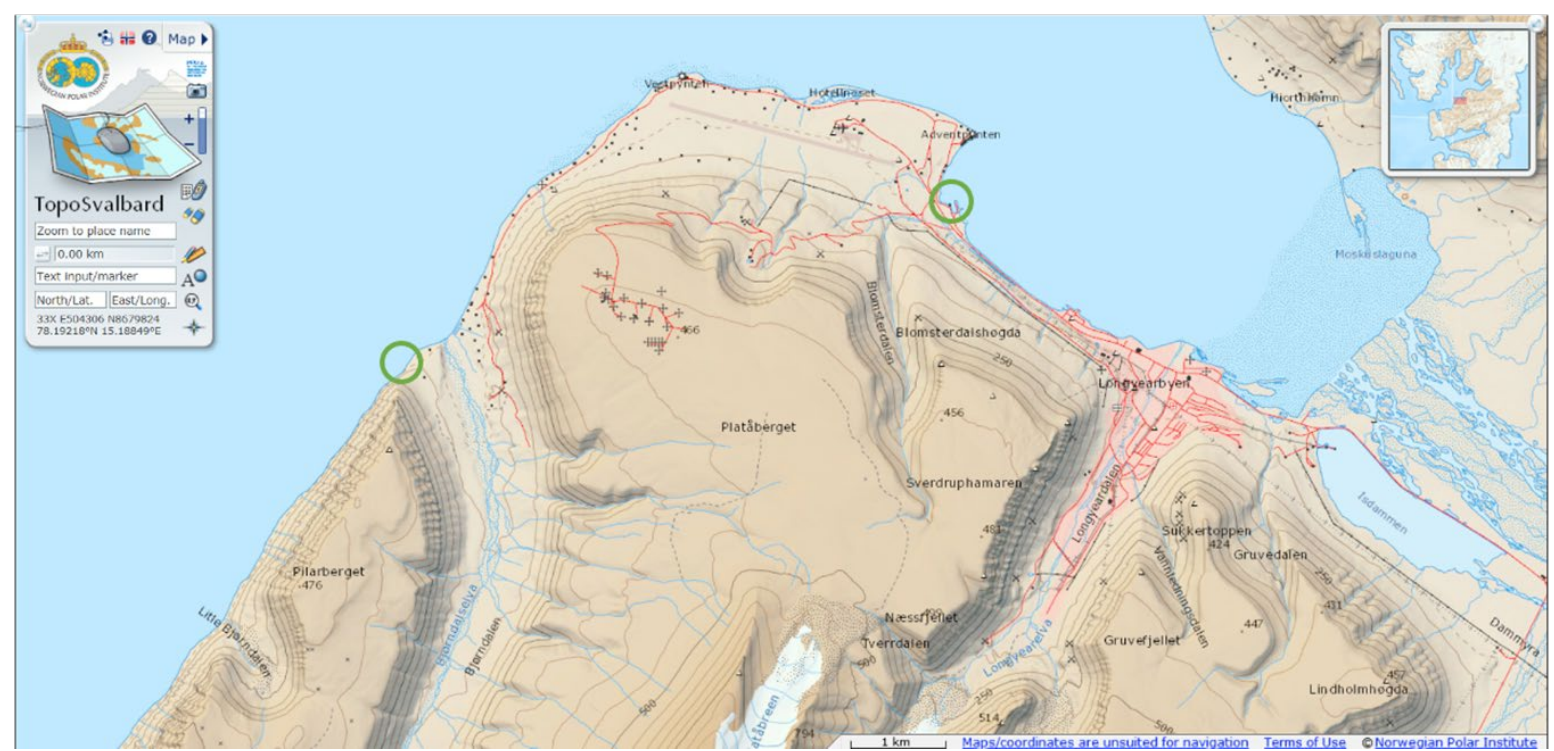




\section{Raudfjorden - Alice Hamna}

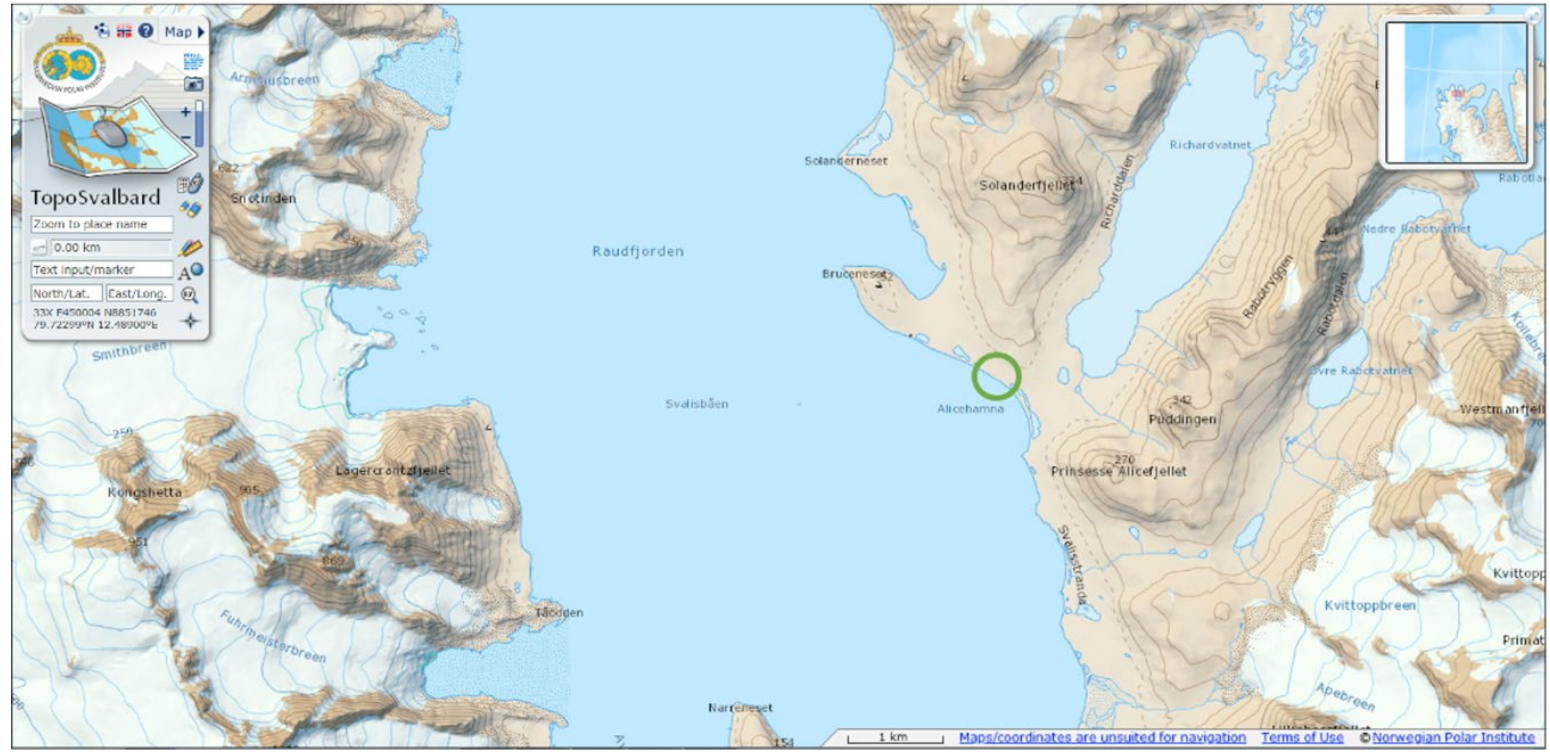

\section{Rijpfjorden - Vindbukta}

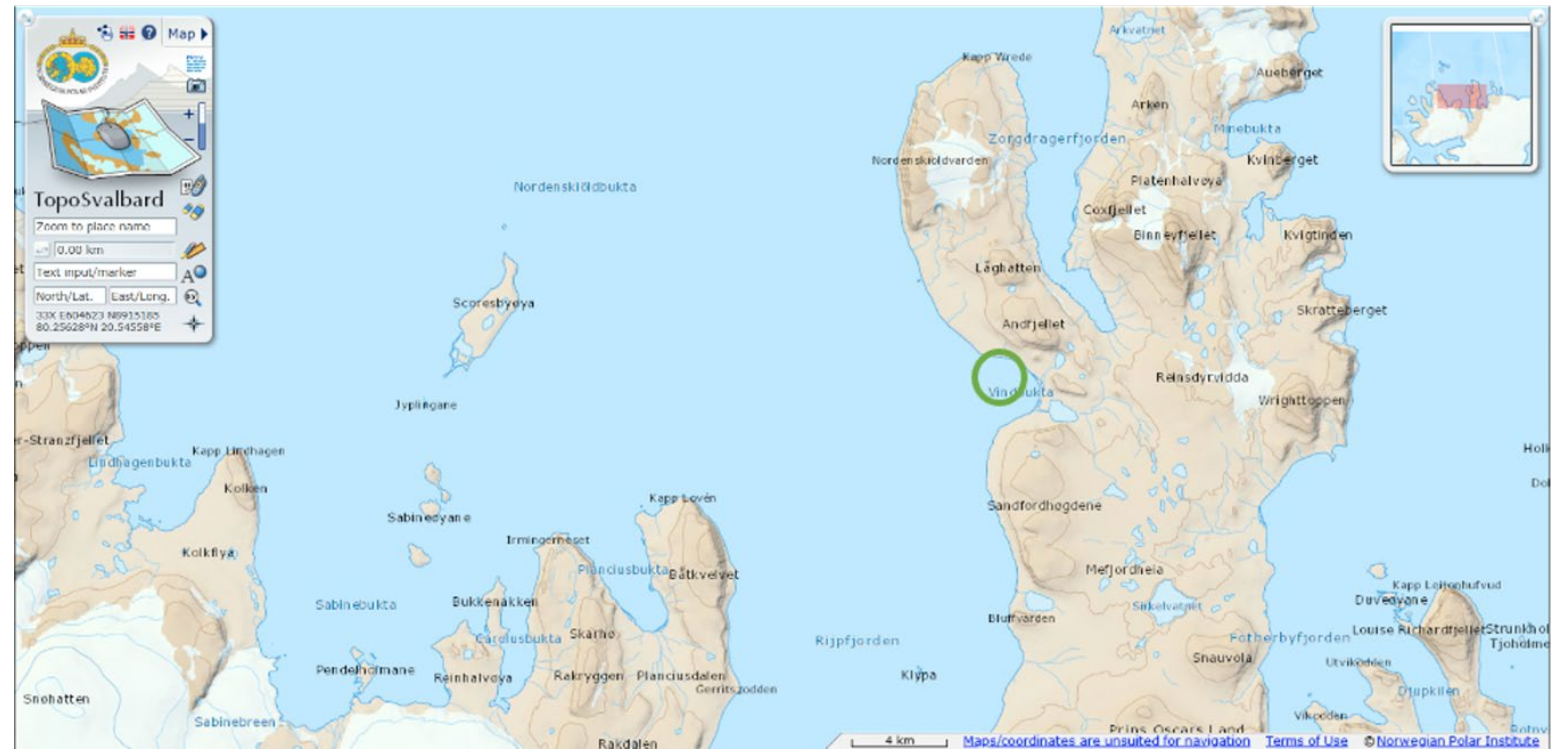




\section{Annex 2. Sampling Protocol}

Total number of samples to be collected per beach:

\begin{tabular}{lll}
\hline Sample type & Number of samples per beach & $\begin{array}{l}\text { Total number of samples } \\
\text { (12-18 beaches) }\end{array}$ \\
\hline Soft sediment - taxonomy macrofauna & 2 tide levels $\times 3$ transects $=6$ & $\mathbf{1 0 8}$ (up to 18 beaches) \\
Soft sediment - taxonomy meiofauna & 2 tide levels $\times 3$ transects $=6$ & $\mathbf{1 0 8}$ (up to 18 beaches) \\
Soft sediment - eDNA & 2 tide levels $\times 2$ transects $=4$ & $\mathbf{7 2}$ (up to 18 beaches) \\
Hard substrate - taxonomy & 2 tide levels $\times 3$ transects $=6$ & $\mathbf{1 0 8}$ (up to 18 beaches) \\
Hard substrate - eDNA & 2 tide levels $\times 1$ transects $=2$ & $\mathbf{3 6}$ (up to 18 beaches) \\
Debris - eDNA & 3 tide levels $\times 1$ transects $=3$ & $\mathbf{4 2}$ (36 samples +6 additional samples \\
& & of interest) \\
\hline
\end{tabular}

Sampling will be conducted preferably at low tide.

Preferred beaches are gradually sloped and consist of sand with presence of rocks.

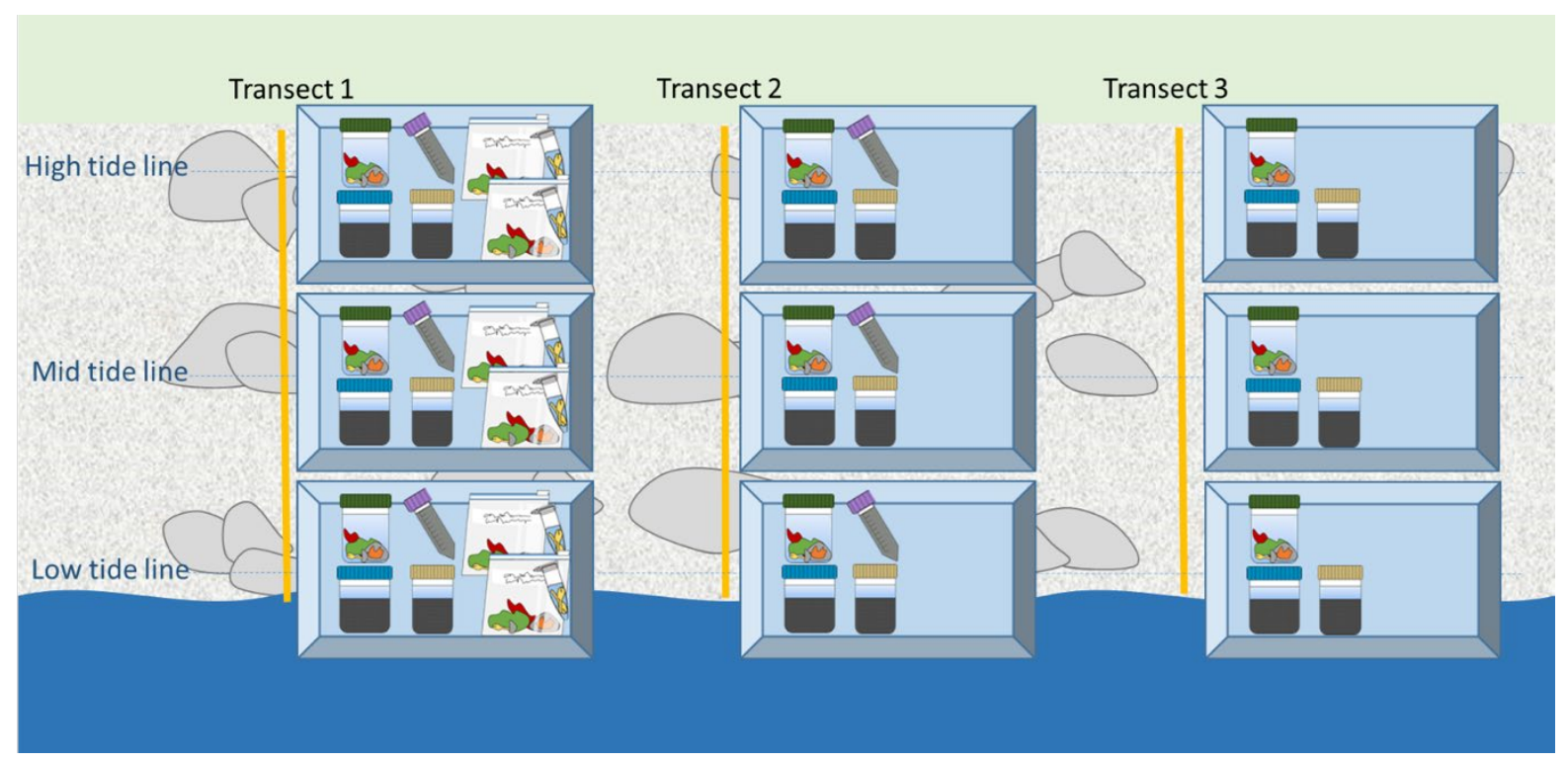

Figure 1. Visual presentation of the number of samples to be collected per beach.

Note: We will sample at two tide levels (not three as initially planned - see figure 1): mid and low tide line. High tide line can still be included if found relevant, for instance on gradually sloped beaches (long slope line with higher chance of finding species at a higher tide mark). 


\section{Materials - all sampling:}

- Tape measure (for measuring transect length)

- Measuring device for salinity and water temperature

- Sediment core $(7 \mathrm{~cm} \varnothing)$

- Steel field sieve $(500 \mu \mathrm{m})$

- Shovel

- Small plastic scoop

- Wash bottle

- Sample jar (plastic, $2.3 \mathrm{~L}$ ) - soft substrate macrofauna

- $37 \%$ formaldehyde (to be diluted to $4 \%$ using a 9:1 dilution with sea water)

- Bengal rose dye (to be added to the $4 \%$ formaldehyde solution)

- Borax (for buffering slightly acidic formaldehyde to a $\mathrm{pH}$ of $\sim 8.2$ )

- Ethanol (>97\%)

- Syringe (diameter, volume and depth?)(from IOPAS)

- Lab gloves (wear while sampling)

- Quadrat $(25 \times 25 \mathrm{~cm})$

- Sediment core $(7 \mathrm{~cm} \varnothing)$

- Steel field sieve $(500 \mu \mathrm{m})$

- HD photo camera

- Paint scraper

- Tweezers

- Sample vials $(50 \mathrm{ml}, 2.5 \mathrm{~mm} \varnothing)$

- Sample bottles - hard substrate taxonomy

- Ethanol (>97\%)

- $37 \%$ formaldehyde (to be diluted to $4 \%$ )

- Bengal rose dye

- Cooler / large sturdy and seawater proof box for storage of samples

- Zip-lock bags

- Stool or foam pad 


\section{Beach description}

Prior to sampling - in the lab (in a fume hood - wear protective gloves!):

- Add 0.4 gram of Bengal rose dye to the $5 \mathrm{~L}$ of $37 \%$ formaldehyde bottle and swirl gently

- Buffer the formaldehyde solution:

Decide how much $37 \%$ formaldehyde you want to add to each plastic carboys (sufficient for each field expedition) and buffer the $37 \%$ formaldehyde per carboy with the necessary amount of borax: $2 \%$ of borax to $37 \%$ formaldehyde solution ( $\mathrm{pH} \sim 8.2$ ).

- First add 21.6 gram of borax (white crystalline compound, use spoon) to the carboy (or the equivalent if less/more than 1 litre of $37 \%$ formaldehyde will be added)

- Add 1 litre of $37 \%$ formaldehyde to the carboy (or the requested amount of $37 \%$ of formaldehyde, equivalent to the amount of borax that is added)

- Mix very gently until the borax has dissolved.

- Check the $\mathrm{pH}$ (should be $~ 8.2$ )

Label the carboy with 'Buffered $37 \%$ formaldehyde + date'.

Cover the label with plastic transparent tape.

Store the carboy safely (in a separate cupboard or outside).

On the beach:

1. Measure salinity and sea water temperature at $1 \mathrm{~m}$ below surface

2. Make an as thoroughly possible description of the beach using the field sheet

3. Take pictures (start with a picture of the name of the beach to enable tracking of the pictures)

4. Identify three locations for a transect along the beach to be sampled.

5. Place the transect from high to low tide at each location and identify two (slowly sloping beaches may have a third one) heights on the beach (low, mid, (high)) to be sampled.

6. Note the time and tide level (high/low tide).

Prepare the sample preservative:

- $\quad$ Either prepare a $4 \%$ buffered formaldehyde solution (by mixing 1 part of $37 \%$ formaldehyde with 9 parts of seawater), that is used as 1:2 mix with sample (1 part of sample with 2 parts of $4 \%$ formaldehyde solution). Mix very gently to evenly distribute the formaldehyde of the sample;

- $\quad$ Or use the $37 \%$ buffered formaldehyde solution by adding $1 / 10$ of the sample volume of the $37 \%$ buffered formaldehyde to the sample. Mix very gently to evenly distribute the formaldehyde of the sample.

\section{Transect 1}

Start with the most promising transect on the beach where both hard and substrate can be sampled. Per transect the following samples will be collected:

\begin{tabular}{|c|c|c|}
\hline Sample type & Tide level & Number of samples per beach \\
\hline 1. Soft sediment - taxonomy macrofauna & Low and mid tide & 2 tide levels $\times 3$ transects $=6$ \\
\hline 2. Soft sediment - taxonomy meiofauna & Low and mid tide & 2 tide levels $\times 3$ transects $=6$ \\
\hline 3. Soft sediment - eDNA & Low and mid tide & 2 tide levels $\times 2$ transects $=4$ \\
\hline 4. Hard substrate - taxonomy & Low and mid tide & 2 tide levels $\times 3$ transects $=6$ \\
\hline 5. Hard substrate - eDNA & Low and mid tide & 2 tide levels $\times 3$ transects $=6$ \\
\hline 6. Debris - eDNA & Low, mid and high & 3 tide levels $\times 1$ transect $=3$ \\
\hline
\end{tabular}




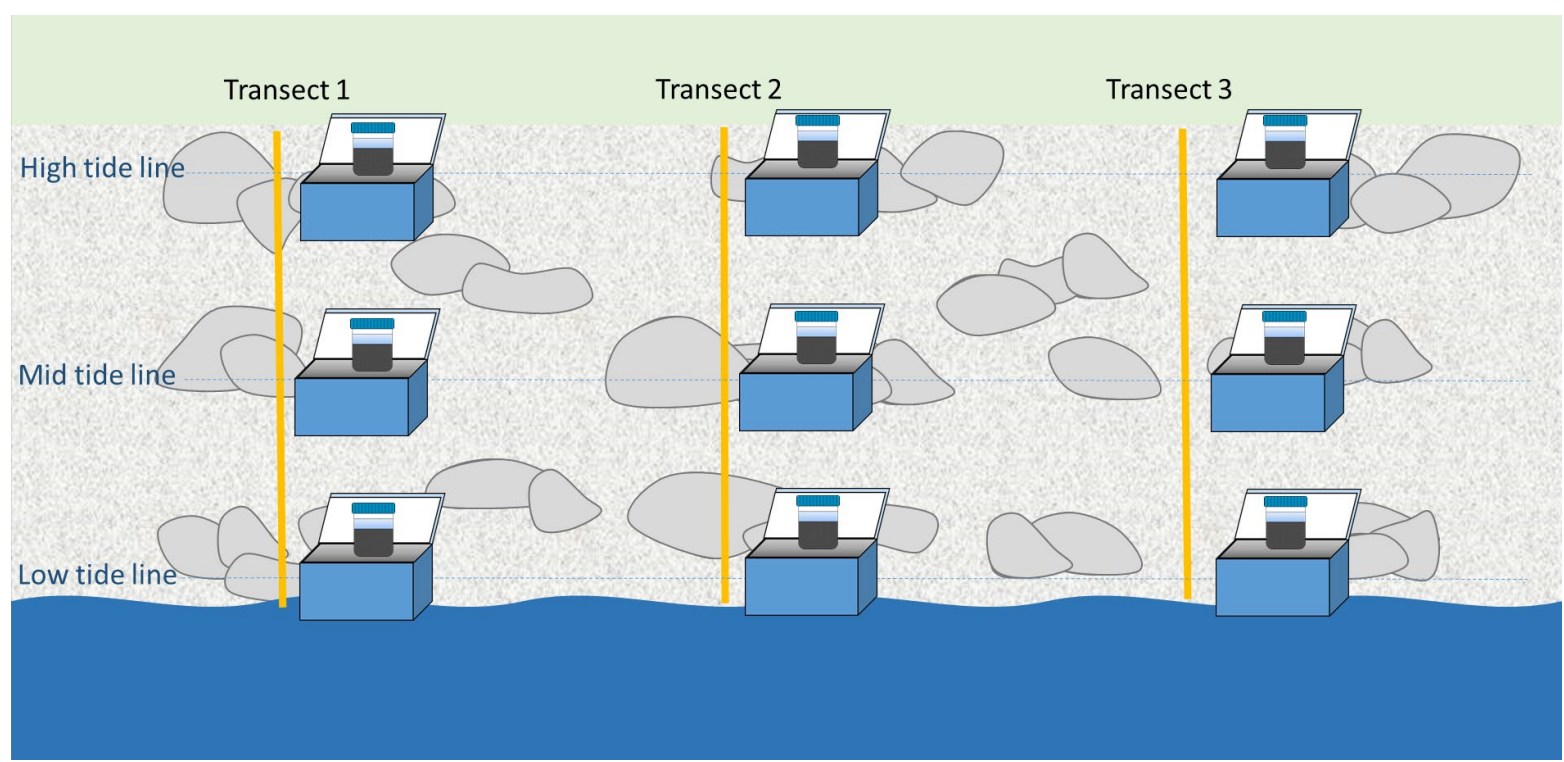

Figure 2. Visual presentation of the macrofauna sampling. Note: We will sample at two tide levels (not three as initially planned - see figure 1): mid en low tide line. High tide line can still be included if found relevant, for instance on gradually sloped beaches (long slope line with higher chance of finding species at a higher tide mark).

Material:

- Tape measure (for measuring transect length)

- $\quad$ Sediment core $(10 \mathrm{~cm} \varnothing)$

- $\quad$ Steel field sieve $(500 \mu \mathrm{m})$

- Shovel

- Small plastic scoop

- Wash bottle

- Sample jar (plastic, 2.3 L) - soft substrate macrofauna

- $4 \%$ or $37 \%$ buffered formaldehyde solution with Bengal rose

Procedure:

1. Take one core sample approximately $10 \mathrm{~cm}$ deep. Close the core if possible and use the scoop to help extract the core without losing any sediment from inside the core.

2. Place the inside of the core on a $500 \mu \mathrm{m}$ sieve.

3. Use ambient sea water to gently dissipate and sieve out the sediment. Make sure no sample material is lost during this process.

4. Use clean water from the wash bottle to move the remainder of the sample material into the corner of a sieve and pour it into a sample bottle. Use the small plastic scoop to help move the material into the bottle, but be careful not to damage any organisms. Use the wash bottle to move any sample material left in the sieve into the bottle.

5. Two options:

a. In case of rather 'dry' samples: add $\mathbf{4 \%}$ buffered formaldehyde/Bengal rose solution to the sample in the bottle (make sure to cover the entire sample completely with the $4 \%$ formaldehyde) and fasten the lid tightly, or:

b. In case of very wet samples: add the more concentrated $\mathbf{3 7 \%}$ buffered formaldehyde/Bengal rose solution to the sample in the bottle (in a volume of $1 / 10$ of the sample) and fasten the lid tightly

6. Store the samples at a dry and not warm place.

7. Repeat for the second tide mark. 


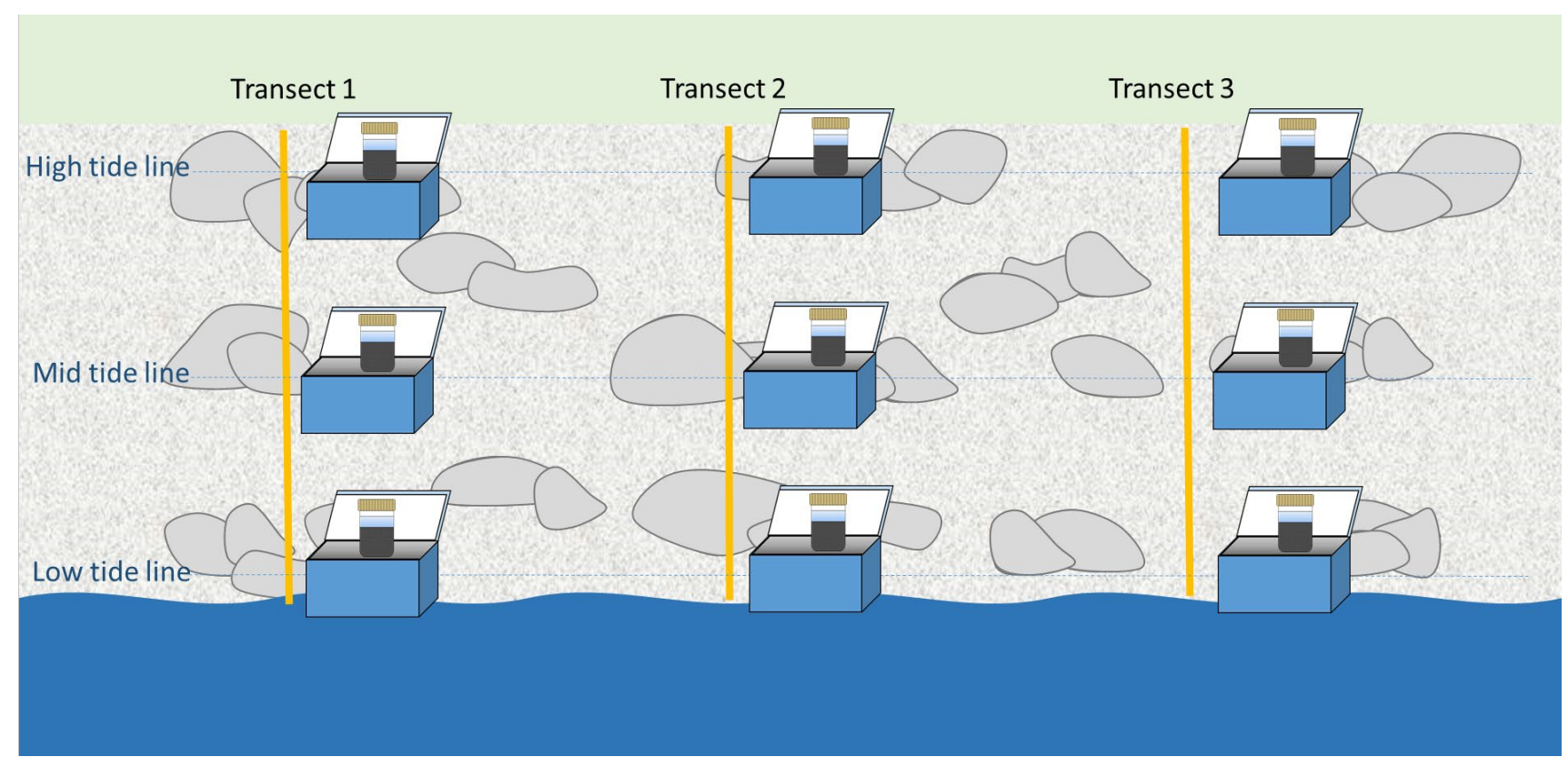

Figure 3. Visual presentation of the meiofauna sampling. Note: We will sample at two tide levels (not three as initially planned and visualised on the figure): mid en low tide line. High tide line can still be included if found relevant, for instance on gradually sloped beaches (long slope line with higher chance of finding species at a higher tide mark).

Material:

- $\quad$ Plexi tubes (to be provided by IOPAS)

- $\quad 50-\mathrm{ml}$ sample jar (provided by IOPAS or the other plastic 50-ml tubes provided by WMR)

- $4 \%$ or $37 \%$ buffered formaldehyde solution with Bengal rose

At each beach:

1. At each height take one plexi tube (or scoop for coarser substrate).

2. Place the contents of the syringe/scoop direct into a 50-ml plastic sample bottle.

3. Two options:

a. In case of rather 'dry' samples: add $\mathbf{4 \%}$ buffered formaldehyde/Bengal rose solution to the sample in the bottle (make sure to cover the entire sample completely with the $4 \%$ formaldehyde) and fasten the lid tightly, or:

b. In case of very wet samples: add the more concentrated $\mathbf{3 7 \%}$ buffered formaldehyde/Bengal rose solution to the sample in the bottle (in a volume of $1 / 10$ of the sample) and fasten the lid tightly

4. Store the samples at a dry and not warm place.

5. Repeat for the second tide mark. 


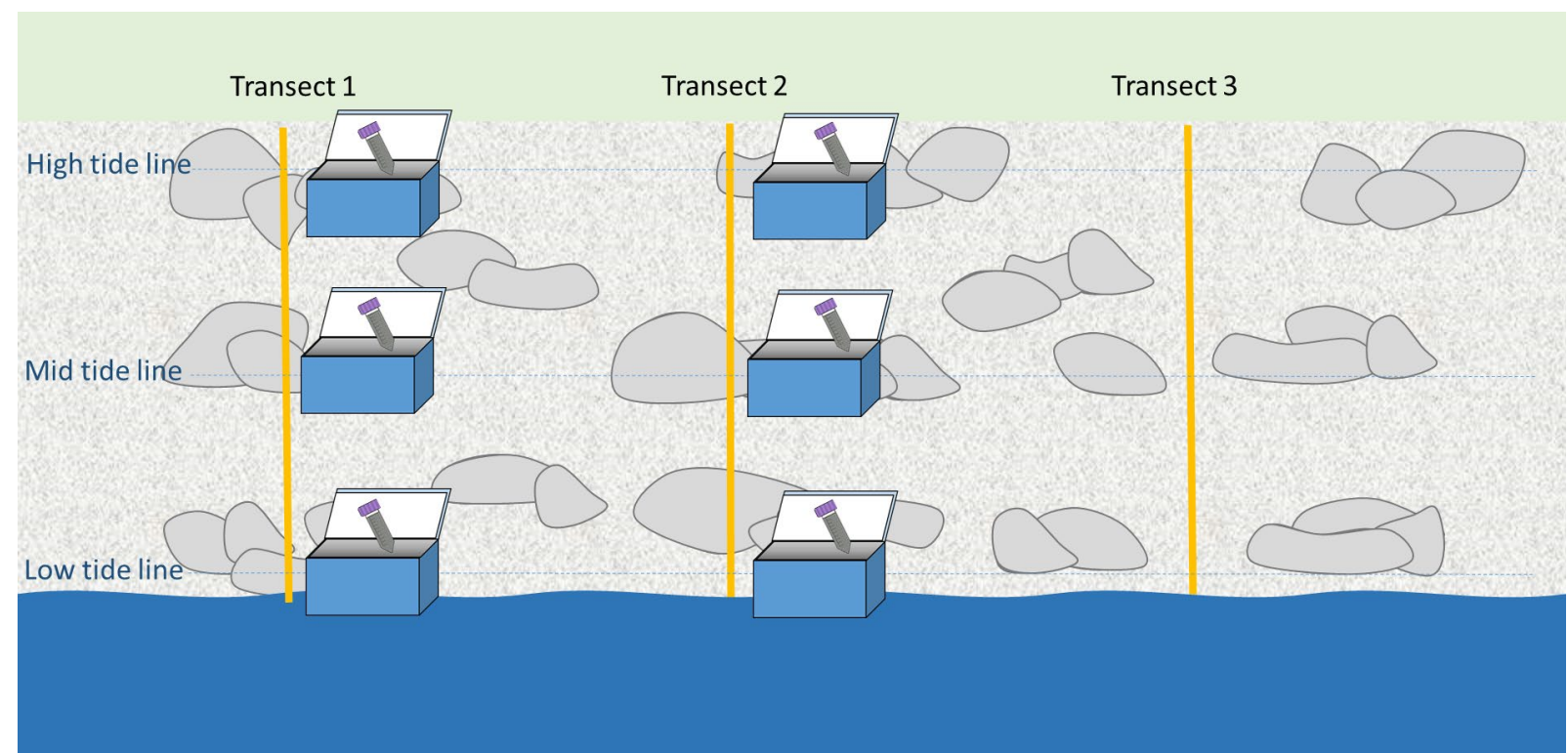

Figure 4. Visual presentation of the eDNA soft sediment sampling. Note: We will sample at two tide levels (not three as initially planned and visualised on the figure): mid en low tide line. High tide line can still be included if found relevant, for instance on gradually sloped beaches (long slope line with higher chance of finding species at a higher tide mark).

Material:

- $\quad$ Sample vials $(50 \mathrm{ml}, 2.5 \mathrm{~mm} \varnothing)$

- $\quad$ Lab gloves (wear while sampling)

Procedure:

1. Scoop the sample vial horizontally through the top layer of the sediment until it is $45 \mathrm{ml}$ full, and fasten the lid tightly. Wipe the outside of the vial clean.

2. Place the sample vials directly in a cooler to keep it cool and out of the sunlight to prevent DNA to degrade. If you do not have a freezer on-board: fill the $50-\mathrm{ml}$ vial a.s.a.p. with $>97 \%$ ethanol and store it cool and in the dark.

3. For each relevant dominant species, place 1-2 individuals (minimum size of a rice grain) into a 2-ml Eppendorf tube or 50-ml plastic tube (depending on size of the specimen), and top this up with $>97 \%$ ethanol. This will be used for taxonomic identification and DNA barcoding. Keep the 2-ml Eppendorf tube cool and out of direct sunlight in a cooler.

4. Repeat for the other tide mark.

5. Back on-board: place the 50-ml sample vial in the freezer a.s.a.p. and the 2-ml Eppendorf tubes in the refrigerator. 


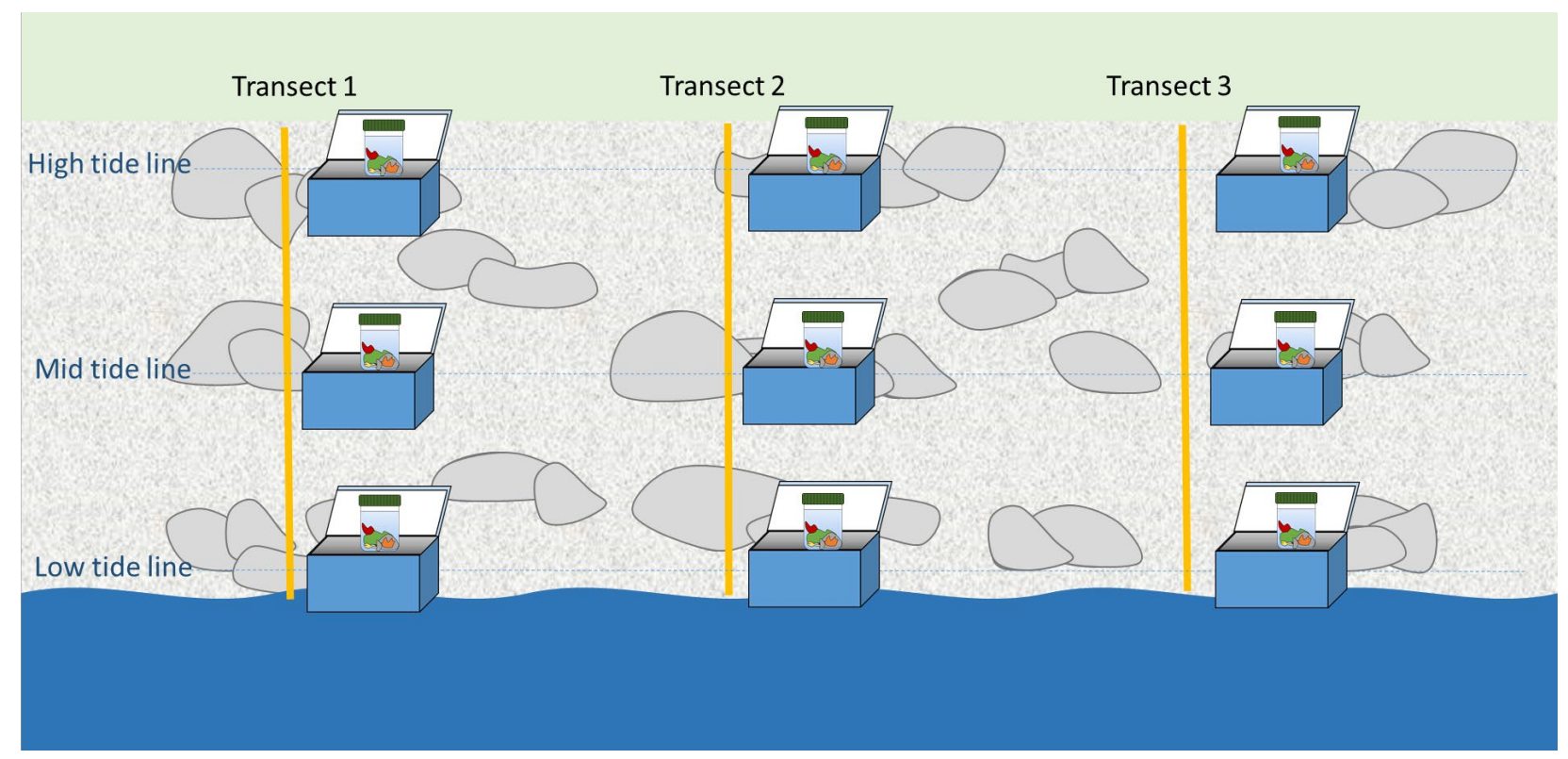

Figure 5. Visual presentation of the hard substrate sampling. Note: We will sample at two tide levels (not three as initially planned and visualised on the figure): mid en low tide line. High tide line can still be included if found relevant, for instance on gradually sloped beaches (long slope line with higher chance of finding species at a higher tide mark).

**The type, amount and distribution of hard substrate present on the beach may dictate the sampling locations, in which case the placement of transects and quadrats can be disregarded.**

Material:

- Quadrat $(25 \times 25 \mathrm{~cm})$

- HD photo camera

- Paint scraper

- Tweezers

- $4 \%$ or $37 \%$ buffered formaldehyde solution with Bengal rose

- 1-2 Litre plastic sample jars (for hard substrate taxonomy)

At each beach:

1. Identify if/what hard substrate is present and record the type, estimated amount and approximate beach height on the field sheet.

2. Place the quadrat flat on the surface (if possible) and take a well-focussed photograph (or several close up) with the quadrat framing the photo.

3. Use (gloved) hands and/or the tweezers to gently remove any organisms within the quadrat and place them in a sample bottle. Organisms attached to small stones or pieces of hard substrate can be placed into sample bottles with the substrate attached.

4. Use the paint scraper to gently scrape off any organisms that cannot be removed with hands or tweezers and place them in a sufficiently sized sample bottle.

5. Two options:

a. In case of rather 'dry' samples: add $\mathbf{4 \%}$ buffered formaldehyde/Bengal rose solution to the sample in the bottle (make sure to cover the entire sample completely with the $4 \%$ formaldehyde) and fasten the lid tightly, or:

b. In case of very wet samples: add the more concentrated $\mathbf{3 7 \%}$ buffered formaldehyde/Bengal rose solution to the sample in the bottle (in a volume of $1 / 10$ of the sample) and fasten the lid tightly

6. Store the samples at a dry and not warm place.

7. Repeat for the second tide mark. 


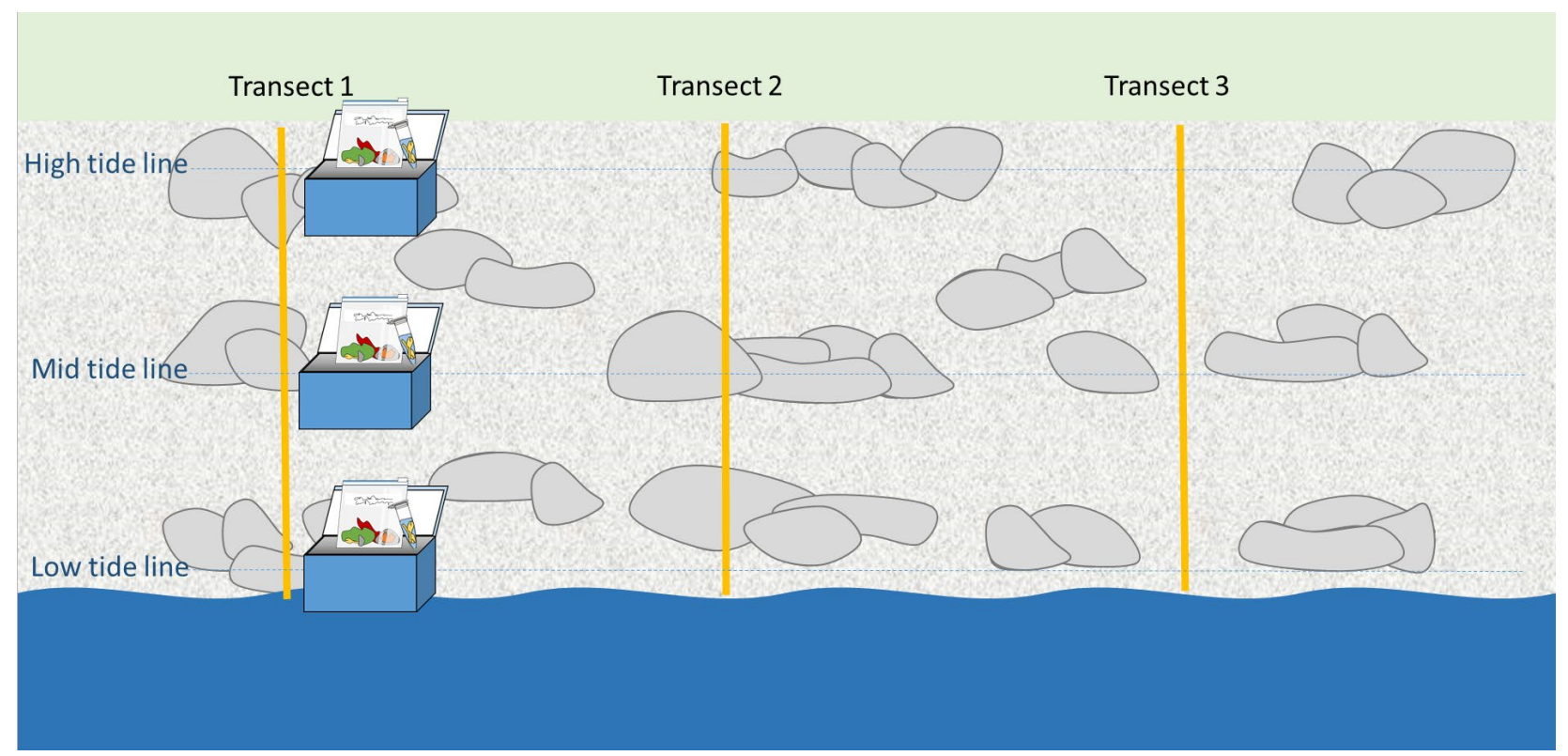

Figure 6. Visual presentation of the eDNA hard substrate sampling. Note: We will sample at two tide levels (not three as initially planned and visualised on the figure): mid en low tide line. High tide line can still be included if found relevant, for instance on gradually sloped beaches (long slope line with higher chance of finding species at a higher tide mark).

**The type, amount and distribution of hard substrate present on the beach may dictate the sampling locations, in which case the placement of transects and quadrats can be disregarded.**

Material:

- Quadrat $(25 \times 25 \mathrm{~cm})$

- HD photo camera

- Lab gloves (wear while sampling)

- Paint scraper

- Tweezers

- Ethanol ( $>97 \%$ ) or $4 \%$ formaldehyde solution with Bengal rose

- Zip-lock bags (for eDNA analysis)

At each beach:

1. Place the quadrat flat on the surface (if possible) and take a well-focussed photograph (or several close up) with the quadrat framing the photo.

2. Use (gloved) hands and/or the tweezers to gently remove any organisms within the quadrat and place them in a zip-lock bag. Organisms attached to small stones or pieces of hard substrate can be placed into the zip-lock bag with the substrate attached. Gently scrape off any organisms that cannot be removed with hands or tweezers and place them in the zip-lock. Keep the zip-lock bag cool and out of the sun in a cooler.

If you do not have a freezer on-board: store the hard substrate sample in a sample jar and fill this a.s.a.p. with $>97 \%$ ethanol. Store it cool and in the dark.

3. For each dominant species, place $1-2$ individuals (minimum size of a rice grain) into a 2-ml Eppendorf tube, and top this up with $>97 \%$ ethanol. This will be used for taxonomic identification and DNA barcoding. Keep the 2-ml Eppendorf tube cool and out of direct sunlight in a cooler.

4. Place the zip-lock bag in the freezer a.s.a.p. and the 2-ml Eppendorf tubes in the refrigerator. 


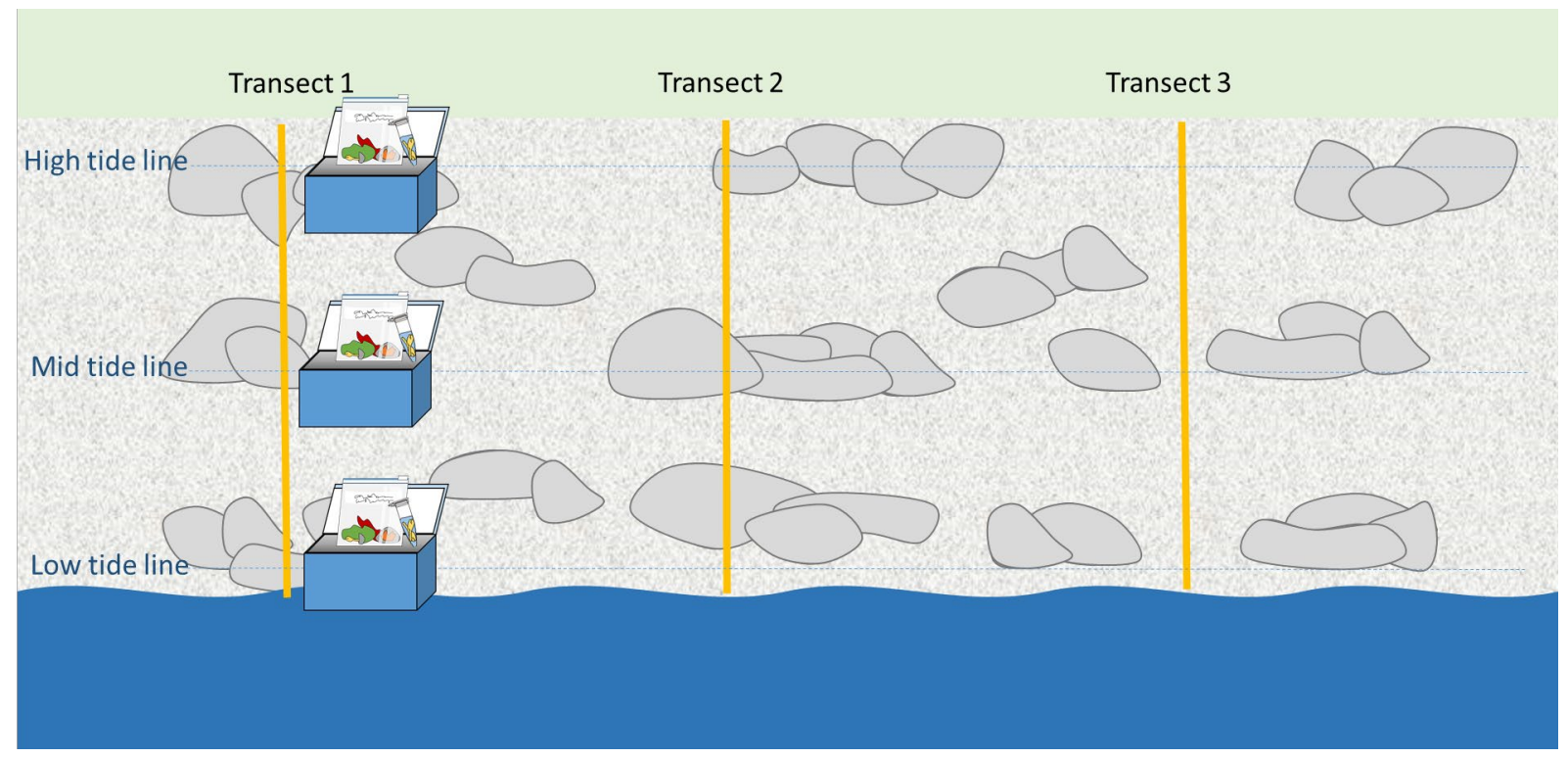

Figure 3. Visual presentation of the debris sampling.

**The type, amount and distribution of debris present on the beach may dictate the sampling locations, in which case the placement of transects can be disregarded.**

Material:

- $\quad$ Tape measure (for measuring transect length)

- HD photo camera

- Zip-lock plastic bags

- 2-ml Eppendorf vials

- $\quad$ Ethanol (>97\%)

- Box cutter

At each beach:

1. Identify if/what debris is present in general and record the type, estimated amount and approximate beach height on the field sheet.

2. Identify three locations along the beach to be sampled.

3. Place the transect from $5 \mathrm{~m}$ above high tide to the low tide mark (if possible) at each location and identify three (if possible) heights on the beach ( $5 \mathrm{~m}$ above to high, mid, low) to be sampled.

4. Spend ten minutes inspecting any debris and if they contain encrusting species along the transect (up to $1.5 \mathrm{~m}$ either side).

5. Take well-focussed, close-up photographs of any organisms attached to the debris.

6. Sample the surface of the debris.

a. Small pieces of debris can be placed in zip-lock bags in their entirety.

b. For large pieces of debris, (sub)sample any encrusted surface by using a box cutter to a $5 \times 5 \mathrm{~cm}$ piece of the encrusted substrate and place it in a zip-lock bag.

c. Any debris surface that cannot be (sub)sampled should be well photographed and the encrusting biota removed using (gloved) hands, tweezers, or a paint scraper and placed in a zip-lock bag.

Keep the zip-lock bag cool and out of the sun in a cooler.

7. For each dominant species, place 1-2 individuals (minimum size of a rice grain) into a 2-ml Eppendorf tube, and top this up with ethanol. This will be used for taxonomic identification and DNA barcoding. Keep the sample bottle cool and out of direct sunlight in a cooler.

8. Place the zip-lock bags a.s.a.p. in the freezer and the 2-ml Eppendorf tubes in the refrigerator. 
Wageningen Marine Research

$\mathrm{T}+31(0) 317487000$

E: marine-research@wur.nl

www.wur.eu/marine-research

Visitors' address

- Ankerpark 271781 AG Den Helder

- Korringaweg 7, 4401 NT Yerseke

- Haringkade 1, 1976 CP IJmuiden
With knowledge, independent scientific research and advice, Wageningen Marine Research substantially contributes to more sustainable and more careful management, use and protection of natural riches in marine, coastal and freshwater areas.
Wageningen Marine Research is part of Wageningen University \& Research. Wageningen University \& Research is the collaboration between Wageningen University and the Wageningen Research Foundation and its mission is: 'To explore the potential for improving the quality of life' 